Louisiana State University

LSU Digital Commons

1993

\title{
Electron-Pair Analysis for Doubly Excited Ridge States.
}

Lijun Zhang

Louisiana State University and Agricultural \& Mechanical College

Follow this and additional works at: https://digitalcommons.Isu.edu/gradschool_disstheses

\section{Recommended Citation}

Zhang, Lijun, "Electron-Pair Analysis for Doubly Excited Ridge States." (1993). LSU Historical Dissertations and Theses. 5553.

https://digitalcommons.Isu.edu/gradschool_disstheses/5553

This Dissertation is brought to you for free and open access by the Graduate School at LSU Digital Commons. It has been accepted for inclusion in LSU Historical Dissertations and Theses by an authorized administrator of LSU Digital Commons. For more information, please contact gradetd@lsu.edu. 


\section{INFORMATION TO USERS}

This manuscript has been reproduced from the microfilm master. UMI films the text directly from the original or copy submitted. Thus, some thesis and dissertation copies are in typewriter face, while others may be from any type of computer printer.

The quality of this reproduction is dependent upon the quality of the copy submitted. Broken or indistinct print, colored or poor quality illustrations and photographs, print bleedthrough, substandard margins, and improper alignment can adversely affect reproduction.

In the unlikely event that the author did not send UMI a complete manuscript and there are missing pages, these will be noted. Also, if unauthorized copyright material had to be removed, a note will indicate the deletion.

Oversize materials (e.g., maps, drawings, charts) are reproduced by sectioning the original, beginning at the upper left-hand corner and continuing from left to right in equal sections with small overlaps. Each original is also photographed in one exposure and is included in reduced form at the back of the book.

Photographs included in the original manuscript have been reproduced xerographically in this copy. Higher quality 6" x 9" black and white photographic prints are available for any photographs or illustrations appearing in this copy for an additional charge. Contact UMI directly to order.

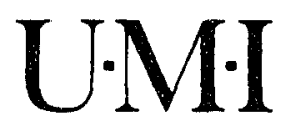

University Microfilms International

A Bell \& Howell Information Company 300 North Zeeb Road. Ann Arbor. MI 48106-1346 USA

313/761-4700 800/521-0600 



\section{Order Number 9401576}

Electron-pair analysis for doubly excited ridge states

Zhang, Lijun, Ph.D.

The Louisiana State University and Agricultural and Mechanical Col., 1993 



\title{
ELECTRON-PAIR ANALYSIS FOR DOUBLY EXCITED RIDGE STATES
}

\author{
A Dissertation \\ Submitted to the Faculty of the \\ Louisiana State University and \\ Agricultural and Mechanical College \\ in partial fulfillment of the \\ requirements for the degree of \\ Doctor of Philosophy \\ in
}

The Department of Physics and Astronomy

by

Lijun Zhang

B.S., Changchun Institute of Optics and Fine Mechanics, 1982

M.S., Louisiana State University, 1988

May 1993 


\section{ACKNOWLEDGEMENTS}

I am deeply grateful to Professor A. R. P. Rau, my supervisor, for his suggestion of this problem, for his close guidance and great patience, and for his editorial help during writing of papers and this dissertation. His advice on scientific research and writing skill has been indispensable for completing this work and will be invaluable to my future scientific career. I have benefited a great deal from his deep insight into atomic physics, his broad knowledge and from his numerous comments and suggestions on this dissertation.

I would like to thank Professors L. H. Chan, J. Callaway, W. Hamilton, D. Temple, J. Tohline and S. Waltkins for serving on my committee and reviewing this dissertation.

My thanks also go to Professors Y. K. Ho and C. Greene for their helpful discussions.

I am grateful to the faculty of the Department of Physics and Astronomy at Louisiana State University (LSU) for their support. Special thanks are due to Karla Tuley, and Gail Spears, the graduate secretaries, for their help. I would like to express my appreciation to Hortensia T. Valdes, the computer consultant, and Monika Lee, the system manager, for their help with the computer facilities.

Thanks are also extended to my classmates, friends, especially to Drs. Hong Gao and Qiaoling Wang, for many helpful discussions and shared ideas, for all the help they offered. Their friendship made my graduate study here more pleasant and memorable. These friendships will be joyfully remembered forever. 
I would like especially to thank my fiance Dr. Aiichiro Nakano for his help and support in preparing this dissertation. His caring and understanding made my last year of graduate study a happiest and most pleasant one.

Finally I owe my special thanks to my family and relatives. I thank my parents who experienced much hardship and brought me up and have been giving me the greatest moral support. I can never forget what my father said "never too old to learn, knowledge is endless". I thank my brothers, sister and aunt for their constant support and encouragement during all the years of graduate study. This dissertation is dedicated to my dear parents and aunt. 


\section{TABLE OF CONTENTS}

PAGE

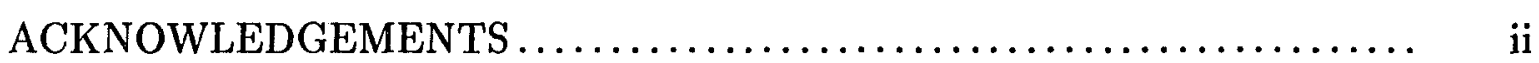

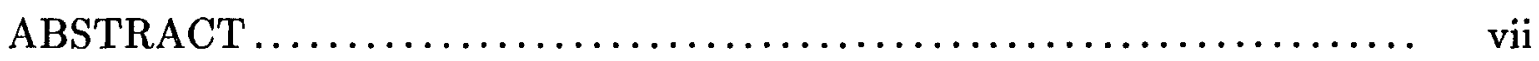

CHAPTER

1 INTRODUCTION $\ldots \ldots \ldots \ldots \ldots \ldots \ldots \ldots \ldots \ldots \ldots \ldots \ldots \ldots \ldots \ldots \ldots, 1$

1.1 The Concept of Doubly Excited States .................. 1

1.2 Experimental Overview ........................... $\quad 2$

1.2.1 Experiments on He ........................ 2

1.2.2 Experiments on $H^{-} \ldots \ldots \ldots \ldots \ldots \ldots \ldots \ldots \ldots \ldots, \quad 3$

1.2.3 Experiments on $\mathrm{He}^{-}$..................... 8

1.3 A General Review of Theoretical Studies ................ 10

2 PREVIOUS STUDIES OF TWO ELECTRON EXCITED STATES

IN $H^{-}$AND HELIUM $\ldots \ldots \ldots \ldots \ldots \ldots \ldots \ldots \ldots \ldots \ldots, 20$

2.1 Stabilization Method $\ldots \ldots \ldots \ldots \ldots \ldots \ldots \ldots \ldots \ldots \ldots \ldots . \quad 20$

2.2 Projection Method............................... 21

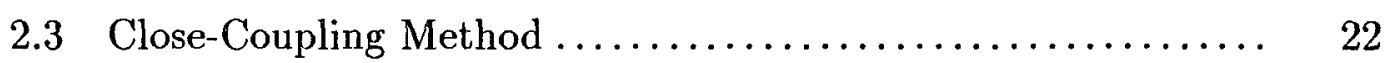

2.4 Complex Coordinate Method $\ldots \ldots \ldots \ldots \ldots \ldots \ldots \ldots \ldots . .24$

2.5 Hyperspherical Method $\ldots \ldots \ldots \ldots \ldots \ldots \ldots \ldots \ldots \ldots \ldots . \quad 25$

2.6 Our Method $\ldots \ldots \ldots \ldots \ldots \ldots \ldots \ldots \ldots \ldots \ldots \ldots \ldots \ldots \ldots \ldots \ldots \ldots \ldots, 27$

3 HYPERSPHERICAL COORDINATES AND SCHRÖDINGER EQUA-

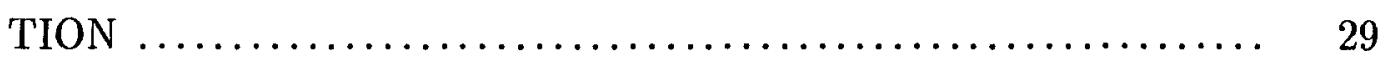

3.1 Hyperspherical Coordinates ...................... 29

$3.2 \quad$ Schrödinger Equation $\ldots \ldots \ldots \ldots \ldots \ldots \ldots \ldots \ldots \ldots \ldots \ldots \ldots \ldots \ldots \ldots \ldots, 31$ 


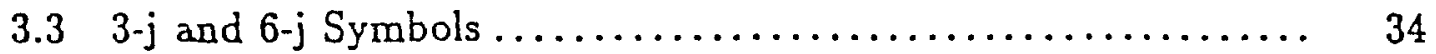

4 ELECTRON-PAIR ANALYSIS FOR DOUBLY EXCITED RIDGE

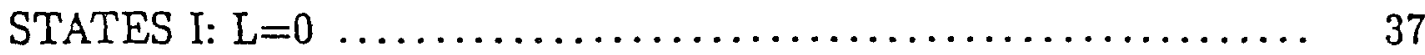

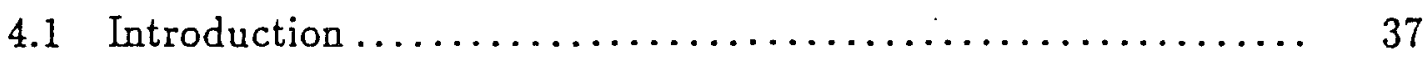

4.2 Pair Analysis of Two Electron States ................ 38

4.2.1 Diagonalization at Fixed $\lambda \ldots \ldots \ldots \ldots \ldots \ldots \ldots \ldots \ldots \ldots \ldots$

4.2.2 Analytical Results for the Extreme Eigenvalues....... 46

4.2.3 An Analytical Pair-Rydberg Formula............. 49

4.2.4 Coupled Potential Wells...................... 52

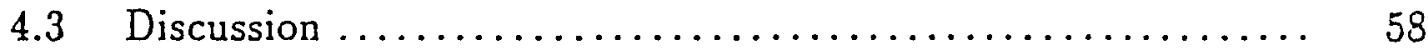

5 ELECTRON-PAIR ANALYSIS FOR DOUBLY EXCITED RIDGE

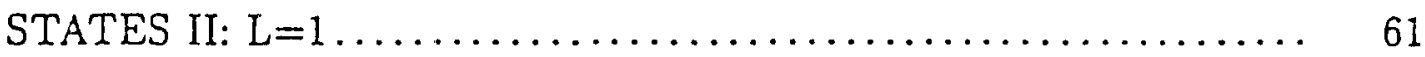

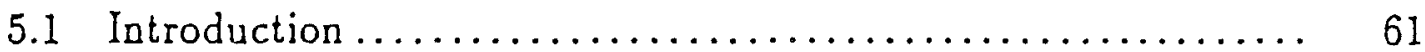

5.2 Calculational Procedure $\ldots \ldots \ldots \ldots \ldots \ldots \ldots \ldots \ldots \ldots \ldots . \ldots \ldots$

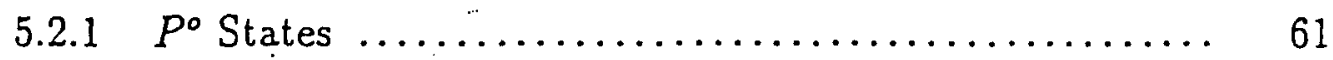

5.2.2 Eigenvalues for ${ }^{1} P^{\circ}$ States ................... 64

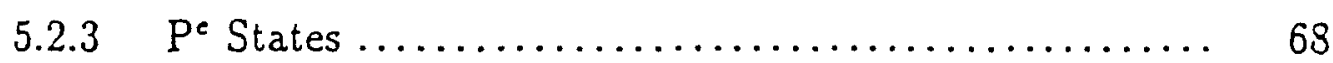

5.2.4 Coupled Potential Wells .................... 72

5.3 Results and Discussion.......................... 79

6 CONCLUSIONS AND FUTURE PROSPECTS $\ldots \ldots \ldots \ldots \ldots \ldots . . \ldots 3$

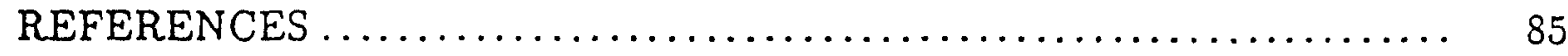


APPENDIX A MAPPING DEGENERATE PERTURBATIONSIN ATOMS ONTO AN ASYMMETRIC ROTOR ............... 94

A.1 Introduction .................................... 94

A.2.1 General Structure.......................... 97

A.2.2 Linear Stark Effect in Hydrogen ................. 99

A.2.3 Diamagnetic Effect in Hydrogen ................. 99

A.2.4 Electron-electron Interaction in Doubly Excited States 103

A.2.5 The Edmonds-Pullen Model .................... 105

A.2.6 Two-nucleons with Quadrupole Coupling............ 105

A.2.7 Summary ................................. 106

A.3 The Asymmetric Rotor at High J .................. 106

A.3.1 Diagonalization of $J_{z} \ldots \ldots \ldots \ldots \ldots \ldots \ldots \ldots \ldots$. $10 \mathrm{~s}$

A.3.2 Diagonalization of $J_{x}^{2} \ldots \ldots \ldots \ldots \ldots \ldots \ldots \ldots \ldots, 112$

A.3.3 The Asymmetric Rotor ...................... 113

A.4 Mapping Problems Onto The Asymmetric Rotor .......... 116

A.5 Summary and Geometrical Interpretation .............. 120

APPENDIX B SOURCE CODE............................. 126

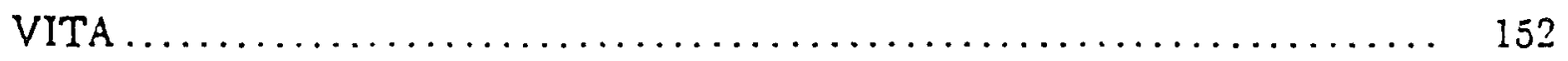




\begin{abstract}
We study doubly excited states of atoms and negative ions by electronpair analysis. The two-electron Schrödinger equation is analyzed in hyperspherical coordinates, with the electrons described throughout as a pair. In contrast to the current adiabatic hyperspherical method, which reverts at large distances to a description in terms of individual electrons, the pair aspect is preserved also asymptotically. Whereas the adiabatic potential wells converge to the single ionization limit, we develop potential wells converging to the double ionization limit of the system, and doubly excited states are then viewed as eigenstates of the pair in these wells. At the simplest level, we get series converging to the double ionization limit which are described analytically by a "Pair-Rydberg" formula, with an effective charge that increases logarithmically with the principal quantum number. In this dissertation, we present the results for ${ }^{1} S,{ }^{1,3} P^{e, o}$ states.
\end{abstract}

Our method consists of first diagonalizing the interaction within degenerate manifolds-here, the three pairs of Coulomb interactions in degenerate manifolds of the so-called "grand angular momentum" in the hyperspherical space. Similar problems involving other interactions in degenerate atomic and nuclear manifolds have also been considered analogously and are presented in an Appendix.

The numerical methods used to solve the Schrödinger equation are Neumann and $5^{\text {th }}$ order Runge Kutta. The computers we used for this work are IBM 3090 and SUN workstation. The computing speed is fast compared with other large scale calculations. On an IBM 3090 it only takes a few minutes 
to get all the potential wells and a few seconds to get an eigenvalue in each potential well. Our method is simple and physically clear. The results are fairly accurate compared with other calculations. 


\section{CHAPTER 1}

\section{INTRODUCTION}

\subsection{The Concept of Doubly Excited States}

The observation and study of doubly excited states started in the 1960s. Doubly excited states mean that two electrons are in excited states. For example, the configuration of the ground state of $\mathrm{He}$ is $1 s^{2}$, but if two

electrons are excited, for instance, as in 2 snp, 2 pns, 2 pnd, $(n \geq 2)$ etc., we have doubly excited states and these are states converging to the $\mathrm{N}=2$ threshold (here, $\mathrm{N}$ represents the principal quantum number of the inner electron and $\mathrm{n}$ represents the principal quantum number of the outer electron).

The first fifty years of the quantum-mechanical study of atoms was dominated by one-electron phenomena. Even in dealing with atoms with many electrons, it was only one active electron that was excited or even, perhaps, ionized. It is only since the 1960 s that the simultaneous excitation of two electrons from the ground state has been studied. Their study has pointed to new phenomena and increasingly to the breakdown of independent electron pictures that have otherwise dominated the field of atomic physics.

Doubly excited states have, therefore, been in the focus of experimental and theoretical research in atomic and molecular physics for the past two decades. The two-electron atoms $\mathrm{He}$ and $\mathrm{H}^{-}$have served as prototypes for this problem. Doubly excited states of atoms (or ions) display the effects of correlations between the two excited electrons. Particularly at high excitation 
of both electrons, their increasing liberation from the dominant central field of the positive ion enhances the importance of the correlations between them. There are two kinds of correlations between two electrons, angular and radial. The angular correlation controls the distribution of the wavefunction in the angle between the two vectors $\vec{r}_{1}$ and $\vec{r}_{2}\left(\vec{r}_{1}\right.$ and $\vec{r}_{2}$ are radial vectors for the two electrons respectively) and originates from the degeneracy in orbital angular momentum of the individual electron states with high principal quantum number $n$. The radial correlation controls the distribution in magnitude of $r_{2} / r_{1}$ and originates from the energy spacing between successive $n$ levels. The study of doubly excited states has, therefore, emphasised the role of angular and radial correlations between the two electrons.

\subsection{Experimental Overview}

There are many experimental techniques to study doubly excited states; for example, electron-atom scattering, photodetachment, photoabsorption, photoionization etc. Recently, high resolution laser and high power synchrotron-radiation light enable us to study doubly excited states for higher $\mathrm{N}$ values.

\subsubsection{Experiments on $\mathrm{He}$ The first observation of a full series} of doubly excited ${ }^{1} P^{\circ}$ helium levels was in 1963 [1]. This observation generated an extensive number of theoretical studies immediately. The observation of doubly excited series by photoabsorption was soon followed by the observation of other doubly excited series by inelastic collision of fast electrons or ions showing analogous results [2]. In the ultraviolet absorption experiments of Madden and Codling, two Rydberg series of ${ }^{1} P^{o}$ levels were found which 
exhibited greatly different decay widths and oscillator strengths. Their experiment $[1,3]$ indicated that among the three possible ${ }^{1} P^{o}$ Rydberg series that converge to the $\mathrm{N}=2$ limit of $\mathrm{He}^{+}$, only one series is prominently observed, while a second series is weakly visible and a third series is completely absent. More experiments have been done afterwards. Woodruff and Samson effectively measured the sum of the photoionization cross section to $2 \mathrm{~s}$ and $2 \mathrm{p}$ states [4]. Later the photoionization experiments for $\mathrm{He}^{+}(\mathrm{N}=2), \mathrm{He}^{+}(\mathrm{N}=3)$, 2s2p ${ }^{1} P$ and $3 \mathrm{~s} 3 \mathrm{p}^{1} P$ states of He have been carried out by different groups [5-10]. In 1989, Zubek et. al. observed autoionizing states of He as resonances in the partial cross sections for simultaneous photoionization and excitation of $\mathrm{He}$, leaving the $\mathrm{He}^{+}$ion in the $\mathrm{N}=2,3$ and 4 states [11]. So far the highest doubly excited states of He observed is up to $N=7$. The most recent work has been done by Domke and his co-workers in 1991 [12] as shown in Fig. 1 and 2. In their experiment, with high resolution $(\Delta E \simeq 6 \mathrm{meV})$ sychrotron-radiation light (the photon energy is in the range of 60 to $80 \mathrm{eV}$ ), they revealed more than 50 states below the $\mathrm{N}=2-7$ thresholds of $\mathrm{He}^{+}$. Their recent experiment on $\mathrm{N}=2$ has reported the observation of all three Rydberg series of ${ }^{1} P^{o}$ resonances accessible by photoionization, including the "missing" $(2 p, n d){ }^{1} P^{o}$ series [13].

\subsubsection{Experiments on $H^{-} \quad$ The lowest lying resonance $2 s^{2}{ }^{1} S$} in $H^{-}$was observed by Schulz in 1964 [14]. Many experiments for other low lying states have been done by different methods [15-18]. After reporting the observation for ${ }^{1} S$ states, Williams and co-workers had observed ${ }^{1,3} P^{o}$ first by electron hydrogen scattering method and they had a series of papers which report the measurements of electron scattering from hydrogen atoms [19]. The basic procedure of their experiment can be described briefly as follows. The 


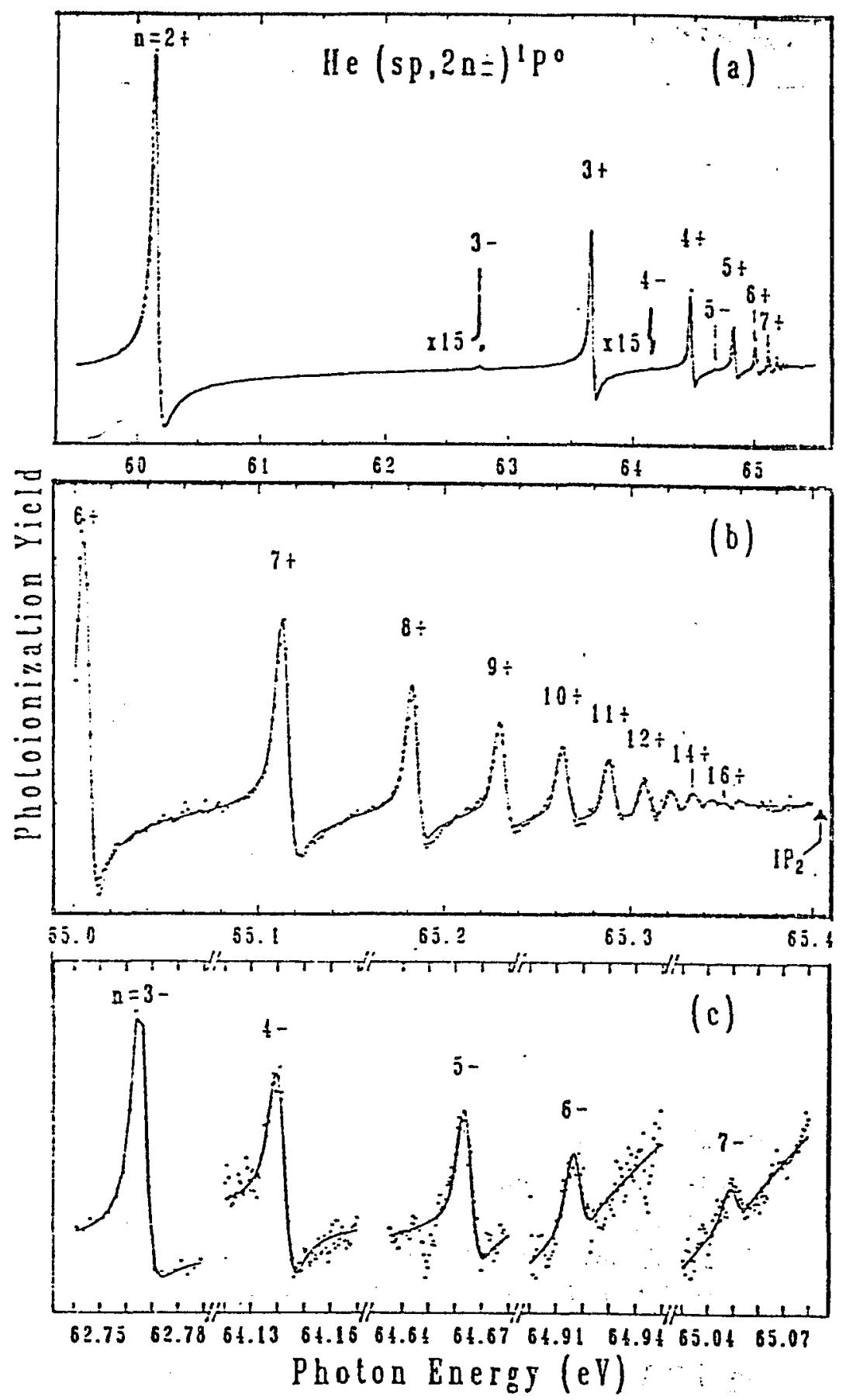

Figure 1. Autoionizing states of doubly excited He below the $\mathrm{N}=2$ threshold $\left(I P_{2}\right)$ of He: (a) overview, (b) magnification of the $n \geq 6$ region, and (c) " $2 n^{-"}$ states (F'ig. 1 of Ref. 12). 


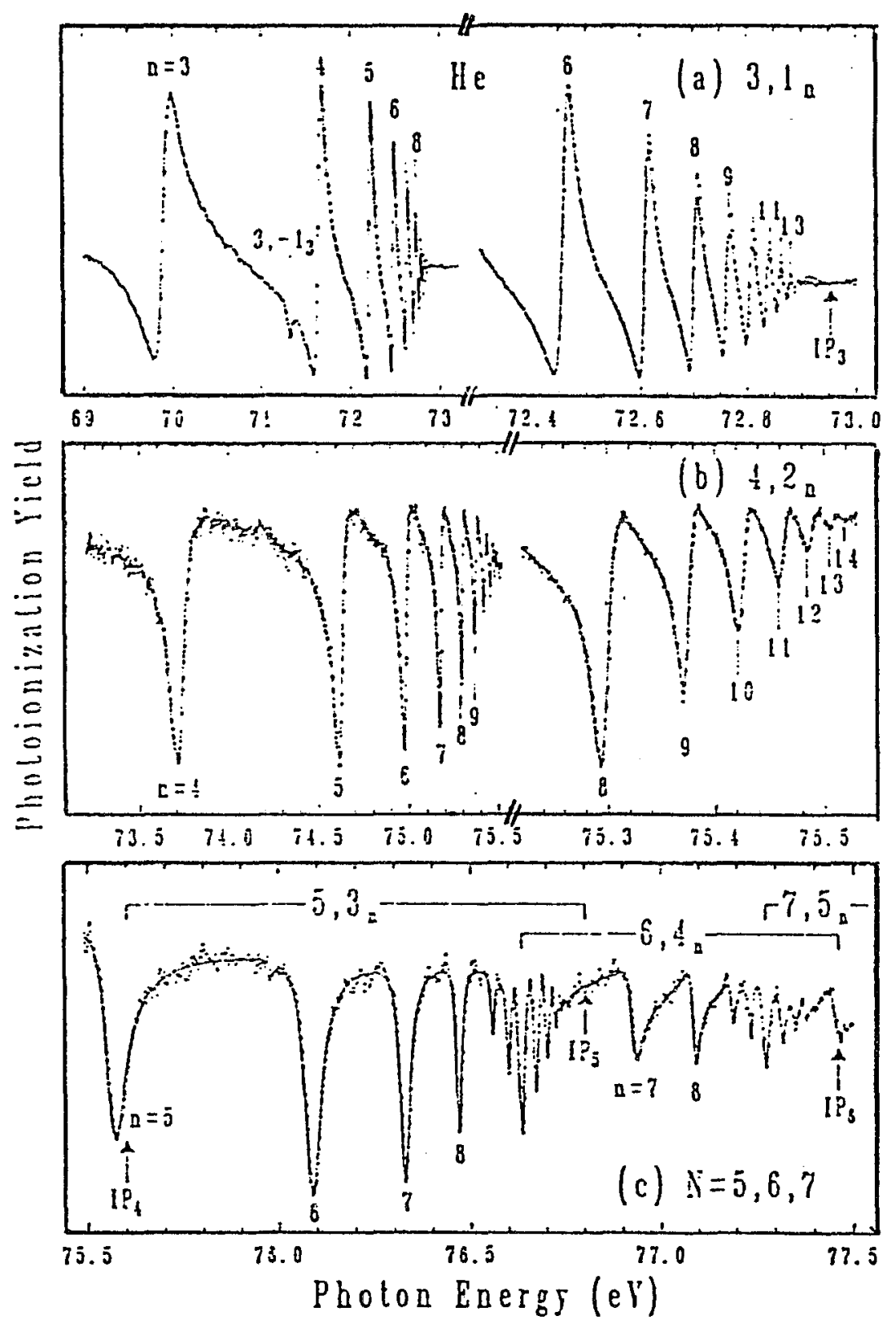

Figure 2. Autoionizing states of He: (a) below the $\mathrm{N}=3$ threshold $\left(I P_{3}\right),(\mathrm{b})$ below the $\mathrm{N}=4$ threshold $\left(I P_{4}\right)$, and (c) below the $\mathrm{N}=5$ and 6 threshold $\left(I P_{5}\right.$, $\left.I P_{6}\right)$. The high-n regions are shown magnified on the right-hand sides in (a) and (b). Note the overlapping of series in (c) (Fig. 2 of Ref. 12). 
electron beam crossed a modulated atomic hydrogen beam. The atomic beam is produced from the thermal dissociation of molecular hydrogen in a tubular tungsten furnace. Detectors were used to detect both scattered electrons and incident electrons. A photon detector was also used to detect emitted photons. The cross sections as the function of the incident electron energy were measured.

More recent experiments using synchrotron or laser light absorption have studied series up to $N=7$. An extremely impressive investigation of resonances was made by the Bryant group [20]. They observed 13 resonance features converging on these thresholds with an absolute uncertainty in position of $1 \mathrm{meV}$ and an energy resolution of about $8 \mathrm{meV}$. Their measurement result is given in Fig. 3. An $\mathrm{H}^{-}$ion beam with relativistic speed was crossed with a laser beam at a varying angle $\alpha$. This changes the photon energy $E_{0}$ into the barycentric photon energy $\mathrm{E}$ in the frame of $\mathrm{H}^{-}$according to the formula

$$
E=E_{0} \gamma(1+\beta \cos \alpha)
$$

where $\beta=v / c$ and $\gamma=\left(1-\beta^{2}\right)^{-1 / 2} ; \alpha=0$ when the beams meet head on. In order to study the high lying resonances in $\mathrm{H}^{-}$, that lie at energies of 10-14 $\mathrm{eV}$, the fourth harmonic of the Nd:YAG laser is Doppler-tuned in this manner to excit the transitions

$$
H^{-}+\gamma \longrightarrow H^{0 *}(\leq N)+e^{-}
$$

where the principal quantum number $\mathrm{N}$ of the hydrogen atom is between four and eight, and the $\leq$ sign indicates that the atom may, instead, be excited to any state lower than $\mathrm{N}$, with the electron carrying off the excess energy. The 

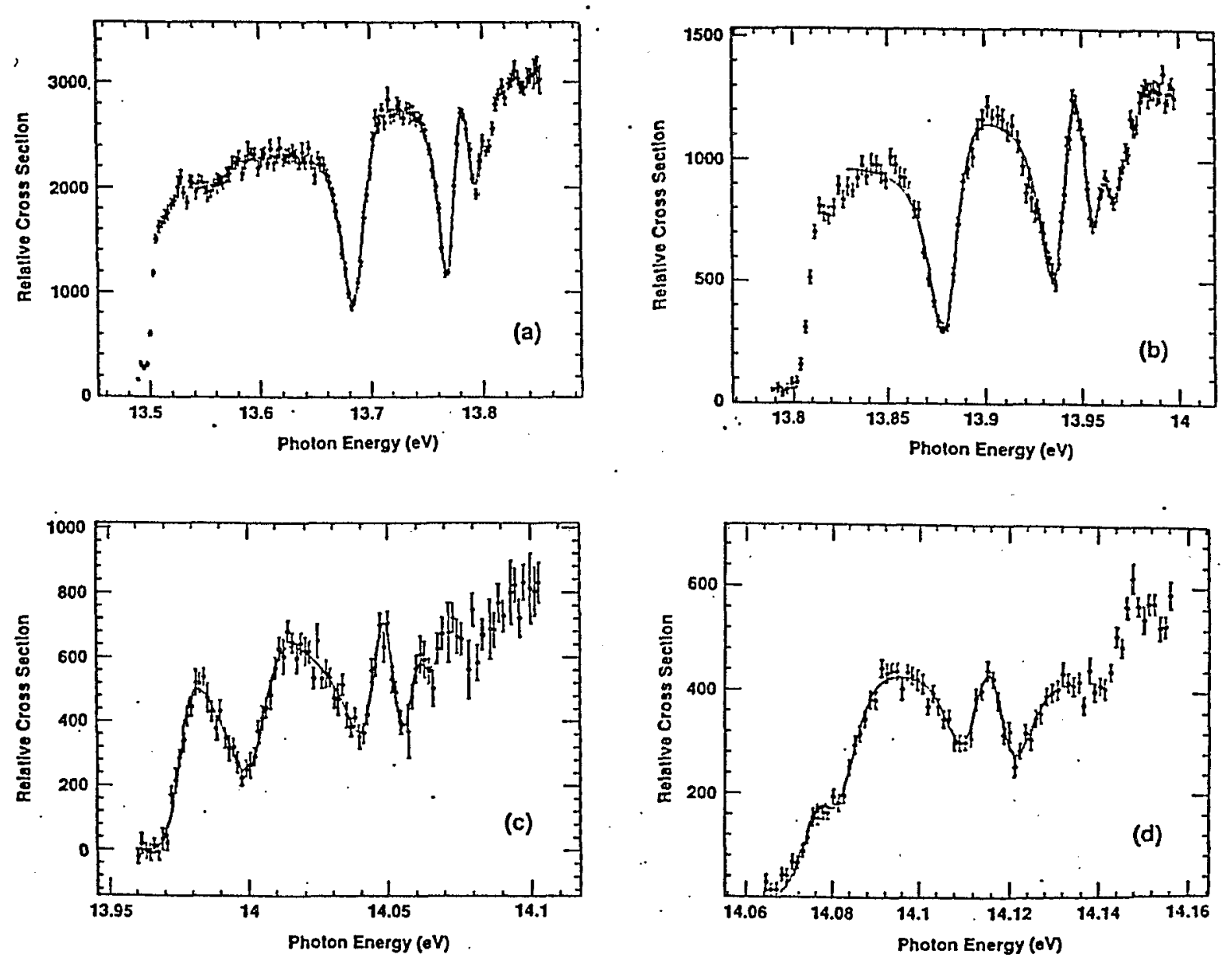

Figure 3. Partial photodetachment cross sections of $\mathrm{H}^{-}$, showing production of neutral hydrogen in (a) $\mathrm{N} \geq 4$; (b) $N \geq 5$; (c) $N \geq 6$; and (d) $N \geq 7$. Intrumental resolution is $8.3 \mathrm{meV}$. Threshold energies as follows: $\mathrm{N}=4,13.5054$ $\mathrm{eV} ; \mathrm{N}=5,13.8084 \mathrm{eV} ; \mathrm{N}=6,13.9746 \mathrm{eV} ; \mathrm{N}=7,14.0748 ; \mathrm{N}=8,14.1398 \mathrm{eV}$ (Fig. 8 of Ref. 20(Harris et. al. 1990)). 
asterisk $(*)$ indicates that the electron in the atom is excited. If the photon energy matches that of a resonance in the $H^{-}$continuum, then the process

$$
H^{-}+\gamma \longrightarrow H^{-* *} \longrightarrow H^{0 *}(\leq N)+e^{-}
$$

may take places. The next step is to measure the production of the neutral hydrogen. Electric field ionization is used to strip $H^{0}(N)$ to proton which are then detected. The Lorentz transformation which converts a magnetic field in the laboratory to a strong electric field sufficient to strip the electron in $H^{0 *}(N)$ is another central feature of the experiment. The protons are magnetically separated from the remaining neutral and positively charged particles. In this way, the production of $H^{0 *}(N)$ state can be measured.

1.2.3 Experiments on $\mathrm{He}^{-} \quad$ The first observation of doubly excited state of $H e^{-} 1 s\left(2 s^{2}\right)^{2} S$ was done by Schulz in 1963 [2]. Other doubly excited states like ${ }^{2} S,{ }^{2} P$, etc. have been also observed by many experimentalists [21-23]. Using electron impact, doubly excites states up to $\mathrm{N}=9$ in $\mathrm{He}^{-}$ have been studied [24]. The first experiment for $n \simeq N>>1$ high doubly excited states was carried out in 1983 [24]. An electron beam of narrow energy spread $(20 \mathrm{meV})$ and variable mean energy is crossed by a beam of helium atoms emerging from a hypodermic needle, and any metastable helium atoms resulting from electron-helium-atom collisions are detected by a channel electron multiplier. The spectrum is as shown in Fig. 4 for such states in the $\mathrm{He}^{-}$ system, as excited by impact of electrons of energy $22-24.5 \mathrm{eV}$ on He, followed by observation of the metastable He atoms into which these states decay. The highest resolved structures correspond to $n \simeq N \simeq 8$.

Ion impact experiments have also observed doubly excited states in positive ions such as $C^{m+}, N^{m+}$, etc., but with $\mathrm{N}$ values less than 4 [25]. 


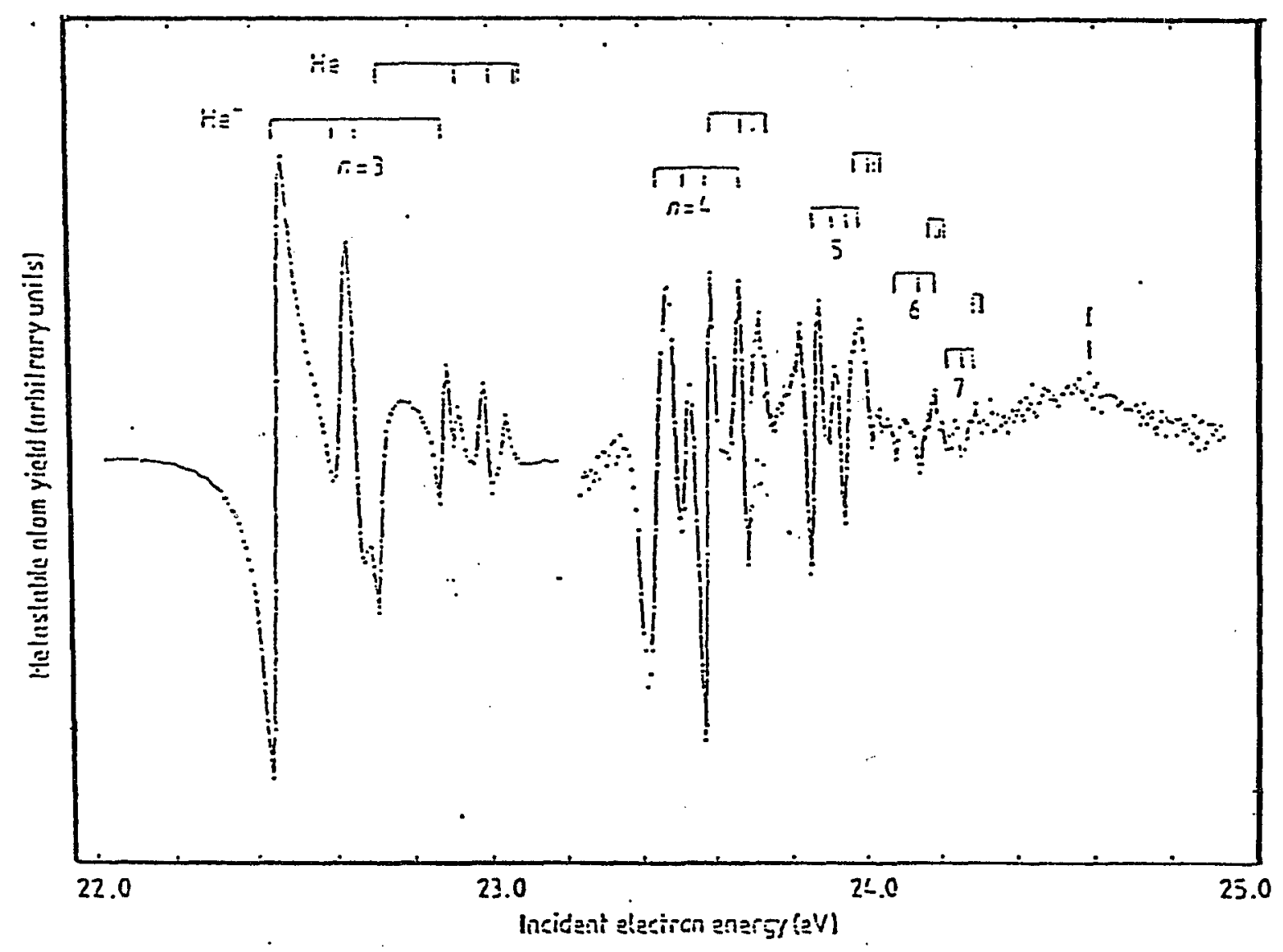

Figure 4. Yield of metastable He atoms following impact of electrons on the ground state of He, showing structure due to doubly-excited states of $\mathrm{He}^{-}$(Ref. 24 (Buckman et. al, 1983)). 


\subsection{A General Review of Theoretical Studies}

An early interpretation of these observations regarded the new doubly excited states in He as superpositions of 2snp and 2pns configurations in nearly equal parts, i.e., quite different from either configuration. This qualitative picture of the correlation between two doubly excited electrons was first provided by Cooper, Fano and Prats [26]. They gave the classification for two-electron excitation levels of He almost immediately following Madden and Codling's experiments. Soon thereafter calculations of the entire spectrum of the doubly excited series of He in the $57-65 \mathrm{eV}$ range were carried out by numerical solution of the two-electron Schrödinger equation. In 1965 Burke and McVicar [27] carried out the numerical solution of the two-electron Schrödinger equation by the close-coupling method. Almost at the same time O'Malley and Geltman [28] did the calculation by variational techniques, and Altick and Moore [29] by a channel mixing method. These calculations have explained the spectral positions, widths and photoexcitation probabilities of numerous doubly excited levels, but have failed to interpret the systematic variation of intensities within each spectrum of given $(L, S)$ and parity and have not clarified the physical basis of the observed regularities. In chapter 2, more recent work with these techniques is described. A new method was needed to interpret the observed regularities. In 1968, Macek [30] studied this problem by using the Fock expansion and its hyperspherical approach, proposing an adiabatic assumption regarding the nature of doubly excited states. This new assumption gave further insight into the origins of the observed regularities, and Macek constructed an approximate calculation based on it. Hyperspherical coordinates for the six variables defining a two-electron atom consist of a radial coordinate $\mathrm{R}$ and five 
angular coordinates $\Omega$. Macek proposed that the two-electron Schödinger equation is approximately separable in the sense that the total wave function may be written as $\Psi \sim F(R) \psi_{\mu}(R ; \Omega)$ where, in the angular function $\psi_{\mu}(R ; \Omega), \mathrm{R}$ is regarded as a parameter. This method was developed much more extensively by $\operatorname{Lin}[31]$.

Hyperspherical coordinates have been used extensively to describe doubly excited states. Other approaches that also treat the pair of electrons together are group theoretic schemes by Kellman and Herrick [32] and Iachello and Rau [33]. Most of this work on doubly excited states to date, both experimental and theoretical, has dealt with the lower end of the spectrum of doubly excited states.

An outstanding problem in the study of doubly excited states is the one that is related to the highly correlated motion on the "Wannier ridge". The concept of the Wannier ridge, promoted by Fano [34] and Rau [35-38], plays an important role in the description of the physics of two-electron escape with zero energy. Its main characteristics are given by Wannier's theory $[34,35,39$ 41]. The central elements of this theory is that the threshold escape of two electrons is dominated by the configuration $\alpha \simeq \frac{1}{4} \pi, \theta_{12} \simeq \pi$, which is a flat saddle point of the potential

$$
V\left(\alpha, \theta_{12}\right)=-\frac{Z}{r_{1}}-\frac{Z}{r_{2}}+\frac{1}{r_{12}}=\frac{1}{R} C\left(\alpha, \theta_{12}\right)
$$

where

$$
C\left(\alpha, \theta_{12}\right)=-\frac{Z}{\cos \alpha}-\frac{Z}{\sin \alpha}+\frac{1}{\sqrt{1-\sin 2 \alpha \cos \theta_{12}}}
$$

and

$$
R=\left[r_{1}^{2}+r_{2}^{2}\right]^{1 / 2}, \quad \alpha=\tan ^{-1}\left(r_{2} / r_{1}\right), \quad \theta_{12}=\cos ^{-1}\left(\hat{r}_{1} \cdot \hat{r}_{2}\right)
$$


The potential (1.4), or equivalently (1.5), displays an infinitely deep valley if $r_{1} \longrightarrow 0$ or $r_{2} \longrightarrow 0$ or, alternatively, $\alpha \longrightarrow 0^{\circ}$ or $90^{\circ}$, and an infinite peak if $\vec{r}_{1} \simeq \vec{r}_{2}$ or, alternatively, $\alpha=45^{\circ}$ and $\theta_{12}=0^{\circ}$. The saddle point occurs at $\vec{r}_{1} \simeq-\vec{r}_{2}$ or, alternatively, $\alpha=45^{\circ}$ and $\theta_{12}=180^{\circ}$. Both valley and saddle regions support discrete or quasi-discrete states $[37,42]$. The saddle point configuration describes the situation when the two electrons have equal radial distances on opposite sides of the nucleus. This is obviously a situation of tight correlation, both in radial excitation and in angles.

It is now clear from many experimental and theoretical studies that doubly excited states fall broadly into two classes [37,42], distinguished by the way the excitation energy is partitioned between the two electrons. The two electrons may either have comparable or disparate excitation. The corresponding states have been named "ridge" and "valley", respectively, and are associated with the ridge and valleys of the potential in (1.4). In the ridge states, there is a complete symmetry and equivalence of both electrons of the pair relative to the residual "grandparental ion" [43]. It is the saddle region that supports the discrete doubly excited ridge states.

These doubly excited ridge states have been analyzed in different ways. In 1977, Read [43] proposed phenomenologically a Rydberg formula for high excitation. He has discussed various modified Rydberg formulae for these states where the two electrons are treated on par in the vicinity of the Wannier saddle point. In 1983, Rau [44] proposed another Rydberg formula. The main point of difference from Read's is in considering the two electrons as a single entity throughout with no reference to quantum numbers for the individual electrons. The experimental results on high doubly excited states of $\mathrm{He}^{-}$provided by Buckman [24] can be well summarized by this Rydberg 
formula. Wang in 1986 [45] introduced another "revised Rydberg formula", and examined both inter- and intra- atomic relationships further. Fits to available data were extended by Molina [46]. Feagin and Briggs [47] and Rost and Briggs [48] presented a diabatic molecular description for these states. The method they proposed is based on a separation in molecular coordinates. They use a trial wavefunction and a variational method to determine eigenvalues in an analytic diabatic potential well.

Configuration interaction, adiabatic hyperspherical and R-matrix expansion [49] methods do not separate the two classes of states. Aymar studied doubly excited states by the method of single electron configurations [50]. In this method, the $L-S$ coupled wavefunction was expanded in terms of independent-particle functions. The eigenvalues were obtained by diagonalizing the two-electron Hamiltonian with certain boundary conditions. Of several configuration interaction calculations, the most accurate are those of Ho and Ho and Callaway [51] using complex rotation techniques.

Nicolaides and co-workers [52], using a judiciously selected symmetrical basis in a standard configuration interaction scheme, have somewhat more extensive results on ridge states. They proposed a method for the identification and accurate calculation of ridge states which lead as a function of excitation energy to the "Wannier state" at the two-electron ionization threshold of any atom in any symmetry. Their theory is state specific and uses suitably defined orthogonal multiconfigurational Hartree-Fock (MCHF) zeroth-order wavefunctions which correspond to the state of lowest energy in each manifold defined by a particular symmetry. The choice of the zeroth-order wavefunction is systematic and allows for the self-consistent computation of the most important angular and radial correlations. 
Other studies of ridge states are based on analytical approximations of the electron-electron interaction [53-57], semi-classical quantization schemes [58-59], the stabilization method [60-62], and Feshbach projection operators [63-68]. We make a few remarks about these other theoretical calculations of doubly excited states. Standard techniques of atomic physics such as the close coupling method $[27,69-70]$ or configuration interaction can be quite successful for the calculation of low lying doubly excited states. Since they involve basis functions that are products of one-electron functions, correlations mix these basis states. Strong two-electron correlations lead to large mixings and, given the diverging number of Coulomb states with increasing excitation, these calculations become impractical for the high lying states near the double ionization limit. Stated in terms of quantum numbers, angular correlations mix different values of $\left(l_{1}, l_{2}\right)$, the individual angular momenta, and radial correlations of $(\mathrm{N}, \mathrm{n})$, the individual principal quantum numbers, of the two electrons. In the limit of extreme correlation, these labels lose meaning and call for a more appropriate set of pair quantum numbers and a corresponding basis. Given the $l$-degeneracy of the Coulomb problem, this feature becomes important for the angular variables already for the low doubly excited states, whether of valley or ridge type. It can be analyzed by considering a fixed $(\mathrm{N}, \mathrm{n})$ manifold and the mixing of different $\left(l_{1}, l_{2}\right)$ states contained in it [71-72]. Models based on the $O_{4}$ group symmetry of this restricted problem have been successful in describing this mixing and in providing alternative pair quantum numbers [73]. At low excitation, the labels $\mathrm{N}$ and $\mathrm{n}$ still retain meaning since states of different $(\mathrm{N}, \mathrm{n})$ are separated in energy. It is now customary to label doubly excited states by these one-electron principal quantum numbers together with $O_{4}$ quantum numbers [74]. On the other hand, at higher excitation, when different $(\mathrm{N}, \mathrm{n})$ 
also lie close in energy and radial correlations mix them, all single electron labels become deficient. This is particularly so for the high ridge states. The words "intra-shell" and "inter-shell" have come into vogue for describing $(\mathrm{N}, \mathrm{n})$ when $\mathrm{N}=\mathrm{n}$, and $N \neq n$, respectively. For low doubly excited states, the former correspond to ridge and the latter to valley states. The latter have also been called "planetary" [75], in analogy with celestial mechanics where individual planets have their own distinct orbits. For the very high doubly excited states, however, the description in terms of intra- and inter- is no longer appropriate given the large mixing of nearly degenerate $(\mathrm{N}, \mathrm{n})$ with both $\mathrm{N}$ and $\mathrm{n}$ large, whether equal or unequal.

A labeling scheme for doubly excited states was first proposed by Herrick in 1975 [76] based on a group theoretical analysis and later was examined by Lin and Macek [77-78]. The pair of quantum numbers $(K, T)$ were introduced to describe the angular correlations between the two electrons as replacement of the pair of $\left(l_{1}, l_{2}\right)$ of the separate orbital quantum numbers. In doubly excited states, because of the electron-electron interaction, the individual orbital quantum numbers $\left(l_{1}, l_{2}\right)$ of the separate hydrogenic orbitals are not constants of the motion. Also not constant are the alternative Lenz vectors $\left(\mathbf{b}_{1}, \mathbf{b}_{2}\right)$, which represent the major axes of elliptical orbits in the classical limit. There are two possible combinations of these vectors which are approximate constants, together with $L$ and $M$; these are

$$
\left|\left(\mathbf{l}_{1} \pm \mathbf{b}_{1}\right)+\left(\mathbf{l}_{2} \mp \mathbf{b}_{2}\right)\right|^{2}=J^{ \pm}\left(J^{ \pm}+1\right)
$$

with $J^{ \pm}$integer or half-integer. The quantum numbers $K$ and $T$ are simply related to $J^{ \pm}$. In this scheme, a given state of a two-electron atom is designated 
by ${ }_{n}(K, T)_{N}^{A}{ }^{2 S+1} L^{\pi}$, where $L, S$ and $\pi$ have their usual meanings, $N$ is the principal quantum number of the inner electron, and $n$ of the outer electron.

The possible values of $K$ and $T$ for any given $N, L$, and $\pi$ are given by

$$
T=0,1,2, \ldots, \min (L, N-1)
$$

and

$$
K=N-1-T, N-3-T, \ldots,-(N-1-T) .
$$

For states where $\pi=(-1)^{L+1}, T=0$ is not allowed. The assigned values of $K$ and $T$ are independent of the spin quantum number $S$. In the zero-order dipole basis, $K$ and $T$ have a physical interpretation. Roughly speaking, $T$ is the projection of the total angular momentum $L$ onto the interelectronic axis and $K$ is proportional to the average value of $r_{<} \cos \theta_{12}$, where $r_{<}$is the radius of the inner electron. The quantum number $A$ was added to emphasize the radial correlation of the electrons [78]. A can take values $-1,0$ and +1 . Both $A=-1$ and $A=+1$ states can have large amplitudes near the potential ridge. the $A=+1$ channel has an antinodal structure at or near $\alpha=\pi / 4$, while the $A=-1$ channel has a node at or near $\alpha=\pi / 4$. Electrons in the $A=0$ states are confined in the two potential valleys. The radial quantum number $A$ depends on the pair quantum number $K$ and $T$. For a given $L, S$, and $\pi$,

$$
\begin{aligned}
& A=\pi(-1)^{S+T}=\pi(-1)^{S+N-K+1} \text { if } K>L-N \\
& A=0 \text { if } K \leq L-N .
\end{aligned}
$$

According to A values, the lowest $\mathrm{n}$ values can be assigned as $n_{\min }=N$ for $A=+1, n_{\min }=N+1$, for $A=-1$ and $n_{\min }=N+1$ for $A=0$. For successive higher $A=0, n_{\min }$ increases by one unit for each $\Delta K=-1$ and 
$n_{\min }$ will remains the same for $\Delta K=0$ and $\Delta T \neq 0$. According to these rules, all intra-shell states have $A=+1$ with $n=N$. Doubly excited states can be uniquely designated in terms of the quantum numbers $K, T, \mathrm{~N}, \mathrm{n}$ and $A$.

Doubly excited states with much higher excitation are less well understood. In this thesis, a method is presented [79-80] which can calculate very high doubly excited states. We will retain hyperspherical coordinates which, as mentioned above, were also used forty years ago for the analysis of threshold escape [39] and in the last twenty years extensively for the study of doubly excited states [30,74,81-83]. These later studies employed an adiabatic approximation which, in analogy to the Born-Oppenheimer method, treats $\mathrm{R}$ as an adiabatic fixed variable while solving the angular part of the Schrödinger equation. Further, they extrapolate the potential wells $U_{\mu}(R)$ at large $\mathrm{R}$ to converge to single ionization thresholds of the atom. This method has successfully described low lying doubly excited states and given insight into the nature of correlations. However, it has also been clear that to proceed to higher excitation will require abandoning the adiabatic separation because the related continuum problem just above threshold demands handling all three variables, $\mathrm{R}$ and angles, on the same footing [39-41]. Also, the adiabatic method, while handling all three-body problems in the collective coordinates for small and intermediate $\mathrm{R}$, departs from them at large $\mathrm{R}$ to return to the independent electron coordinates for the asymptotic region. Thereby doubly excited states are viewed as successive groups that converge to individual ionization thresholds of a "parent" ion formed by (ion + e). Having one electron remain bound, say in a principal quantum number $\mathrm{N}$, while the other runs through successive values of $n=(N, N+1, \ldots \infty)$ for the group of doubly excited states below 
this Nth single ionization threshold means that asymptotically the description is suited to the valleys of the potential surface $: r_{2} / r_{1} \longrightarrow 0$ or $\infty$, that is, $\alpha \longrightarrow 0$ or $\pi / 2$, as $R \longrightarrow \infty$. Our primary point of departure from the adiabatic hyperspherical method is that we do not revert at any stage to independent electron coordinates or single ionization thresholds. We diagonalize the operator $\mathrm{C}$ in (1.5) within manifolds of the operator $\mathbf{\Lambda}^{2}$ and thereby get potential wells that converge to the double escape threshold. We retain in this manner a pair description throughout. We also use the same labeling scheme as mentioned before.

Apart from studying doubly excited states, this thesis work also involved the study of several problems in atomic physics, such as diamagnetism and the Stark effect in high Rydberg states and analogous problems at high excitation in nuclear physics or in other branches of physics, which display common features with doubly excited states [84]. Among these are the existence of classes of sharply localized states, such as the ridge states, formed through large superpositions of basis states that are degenerate in the absence of the interaction. The asymmetric rotor at high angular momentum $J$ also displays these same features: In particular, most of its eigenstates divide into two groups with different localizations (along the directions of minimum and maximum moments of inertia). A few states lie in a "separatrix" region in between, corresponding to localization along the axis with an intermediate moments of inertia. A 1:1 mapping is made from the atomic and nuclear problems into the asymmetric rotor, wherein the principal quantum number $n$ and orbital angular momentum $l$ of the former are put in correspondence, respectively, with $J$ and $M$, the azimuthal projection of the rotor's angular 
momentum. For each given problem, the "asymmetry parameter" of the corresponding rotor is identified, and eigenvalues and eigenfunctions are presented. The key, common feature that underlies localization is the vanishing as $l \longrightarrow n$ of the off-diagonal coupling between the states in a degenerate manifold $\{n l\}$ just as in the asymmetric rotor where the matrix elements of the step-up and step-down operations in the $\mid J M>$ basis vanish when $M \longrightarrow \pm J$.

Chapter 2 of this dissertation review the previous study of doubly excited states briefly and also give the foundermental idea of our method. Chapter 3 of this dissertation presents the two-electron problem, more details on the coordinates used and the resulting two-electron Schrödinger equation and some other preliminaries to be used in the following chapters. Chapter 4 presents our method of solution and results of this pair analysis for doubly excited ridge states with $L=0$, and chapter 5 extends the work to $L=1$. Chapter 6 consists of conclusions and discussions. In appendix A, we discuss the mapping of degenerate perturbations in atoms onto an asymmetric rotor. In appendix B, we list some of the source codes used in this thesis. Throughout this thesis, we use atomic units $(\hbar=m=e=1$, unit of length : Bohr radius $a_{0} \simeq 5 \mathrm{~nm}$, unit of energy : $e^{2} / a_{0} \simeq 27.2 \mathrm{eV}$ ). 


\section{CHAPTER 2}

\section{PREVIOUS STUDIES OF TWO ELECTRON EXCITED STATES IN $H^{-}$AND HELIUM}

\subsection{Stabilization Method}

The stabilization method is one of the methods for calculating resonance parameters. It has been used to calculate resonance energies $E_{\gamma}$ and the decay width $\Gamma$ by many people [60-62]. The basic idea of this method is follows : (a). choose an appropriate basis of square-integrable functions, (b). diagonalize the complete Hamiltonian in successively larger bases, (c) for sufficiently large basis sets, the presence of a "stable" root indicates a resonance, and the degree of stability of that root is a measure of the width. For an excited atom with internal states at energies $E_{i}, \mathrm{i}=1,2 \ldots$, the complete wavefunction may be expanded, similarly to close-coupling,

$$
\Psi_{E}=A \sum_{t=1}^{\infty} \varphi_{t}\left(\vec{r}_{0}\right) F_{t}(\vec{r})
$$

where $\vec{r}_{0}$ represents the coordinates of the target particles, $\vec{r}$ represents the coordinates of an external electron, $\mathrm{A}$ is the antisymmetrization operator and $\mathrm{E}$ is the total energy. For specific energies the channel function $F_{t}(\vec{r})$ are expanded in terms of a set of square-integrable basis function $u_{m}(\vec{r}), F_{t}(\vec{r})=$ $\sum_{m=1}^{M-t} u_{m}(\vec{r}) c_{m t}^{(j)}$. By diagonalizing the complete Hamiltonian in the basis $\Psi_{E}=$ $\sum_{t=1}^{T} \varphi_{t}\left(\vec{r}_{0}\right)\left(\sum_{m=1}^{M_{t}} u_{m}(\vec{r}) c_{m t}^{(j)}\right.$, one can get the specific energies $\epsilon_{j}$ and expansion 
coefficients $c_{m t}^{(j)}$. After finding the stable root the eigenfunction corresponding to a stable eigenvalue $\epsilon_{j}$ can be used to calculate the resonance parameters. The major assumption made in stabilization method is the basis used is very large in order to span the range of the potential involved in the problems.

\subsection{Projection Method}

The projection operator method was proposed by Feshbach for studying nuclear problems in the early 1960s [85] and has been extensively used to calculate elastic and inelastic scattering problems and autoionization resonances for $H^{-}$and He [63-68] since then. In the Feshbach formalism the Schrödinger equation becomes

$$
\left(P H P+P H Q \frac{1}{E-Q H Q} Q H P-E\right) P \Psi=0,
$$

where $\mathrm{P}$ and $\mathrm{Q}$ are the open and closed channel projection operators, respectively. $Q$ and $P$ satisfy following relations

$$
\begin{aligned}
P+Q & =1 \\
P^{2} & =P \\
Q^{2} & =Q
\end{aligned}
$$

and

$$
Q^{(N)}=Q_{1}^{(N)} Q_{2}^{(N)}
$$

Here the superscript $\mathrm{N}$ indicates the projection operators relevant to the 
autoionization states just below the Nth hydrogenic threshold. Using the eigenfunction $\phi_{s}$ of $Q H Q$, we have

$$
\left[Q H Q-\epsilon_{s}\right] Q \phi_{s}=0
$$

The exact resonance position $\left(E_{s}\right)$ is given by

$$
E_{s}=\epsilon_{s}+\Delta_{s}
$$

and the width $\Gamma_{s}$ is defined by

$$
\Gamma_{s}=2 \pi<\phi_{s}|Q H P \delta(E-P H P) P H Q| \phi_{s}>
$$

The continuum of $Q H Q$ starts at the hydrogenic threshold $\mathrm{N}$ and all eigenvalues below this continuum correspond to the autoionization levels described to the first order by equation (2.2). The autoionization energies can be determined by using the Rayleigh-Ritz variational procedure. There are several ways to choose the trial function $\phi_{s}$. Once the trial function $\phi_{s}$ is decided, one can find the upper bound on the exact eigenvalues $\epsilon_{s}$ by diagonalizing the operator $Q H Q$. The correction of the eigenvalues can be determined by

$$
\Delta_{s}=<\phi_{s}\left|Q H P \wp(E-P H P)^{-1} P H Q\right| \phi_{s}>
$$

So with appropriate trial function $\phi_{s}$, one can find resonance energies $E_{s}$ by using equation (2.6).

\subsection{Close-Coupling Method}

The close-coupling method was used in atomic physics for calculating cross-sections for electron impact with hydrogen and other atoms $[27,86]$. The starting point of this method is to expand the wavefunction in terms of some 
target form and the function representing the scattered electron. In electronhydrogen collisions case, the target states are eigenstates of the hydrogen atom. The close coupling approximation means that only a few low-lying states are included and all continuum states and highly excited bound states are excluded from the expansion. Complicated integro-differential equations are obtained which may be numerically integrated by an iterative method. Cross sections can be calculated once the knowledge of the asymptotic behavior for the channel wave function $F_{i}$ are obtained. However, the convergence of this method was very slow because other contributions were ignored. Burke and co-workers have modified the usual form of the close-coupling method by including in the eigenstates expansion pseudostates chosen according to some rules.

This method has been extensively used by Callaway et. al. with K-matrix and pseudostate calculations $[66,87]$. In early of their work, they used 3 states (1s-2s-2p) close coupling and calculated the resonance energies and cross sections below $N=2$ threshold with variational procedure [88]. The later studies, they included pseudostates which were allowed to be either closed or open to the atomic states. In their recent work, they employed a big basis which contained 10 exact atomic states and 18 pseudostates, and studied doubly excited states up tp $N=4$.

The wavefunction of the system Hamiltonian has the form

$$
\Psi\left(\Gamma_{j}, \vec{x}_{1}, \vec{x}_{2}\right)=(2)^{-1 / 2}\left(1-P_{12}\right) \sum_{\gamma_{i}} \psi\left(\Gamma_{i}, \vec{x}_{1}, \hat{r}_{2}, \sigma_{2}\right) F_{i j}\left(r_{2}\right),
$$

in which $\vec{x}_{i}$ denotes the space and spin coordinates $\left(\vec{r}_{i}, \sigma_{i}\right)$ of particle $i$; the quantum number $\Gamma_{i}$ includes the conserved quantum numbers describing the total orbital angular momentum, spin, and parity; the orbital angular momentum of the atomic state and of the scattered electron; and all other numbers 
required to determine the atomic state. The incident channel is denoted by $\Gamma_{j}$. The operation $P_{12}$ interchanges coordinates and spins of both electrons. The wavefunction $F_{i j}$ describes channel $i$ when an electron is incident in channel $j$ and obeys the specified boundary conditions. $F_{i j}$ is represented as

$$
F_{i j}=\alpha_{0 i} S_{0}\left(k_{i}, r\right)+\alpha_{1 i} S_{1}\left(k_{i}, r\right)+\Phi_{i j}(r)
$$

where The $S$ are functions with specified asymptotic forms and $\Phi_{i j}$ is a normalizable function which contains the long-range and short-range correlation functions. The summation also include pseduostates which contain contributions from continuum, and/or a number of correlation terms that include short range dipole interactions or long range quadrupole interactions. Using variational method, one can derive the integro-differential equation for $F_{i j}$. The K-matrix defined in Ref. 88 is real and symmetric, and is related to the $\mathrm{S}$ matrix by $\mathrm{S}=(1+\mathrm{iK}) /(1-\mathrm{iK})$. K-matrix can be determined by solving for $F_{i j}$. Therefore, cross-sections are computed from the element of $\mathrm{S}$ by standard procedures [88].

\subsection{Complex Coordinate Method}

The complex coordinate rotation method is based on the mathematical developments of Aguilar, Balslev and Combes [89-90] and Simon [91]. The basic idea of this method is a frame transformation. In the complex rotation method, all the inter-particle coordinates $r_{i j}$ are transformed by

$$
r_{i j} \longrightarrow r_{i j} e^{i \theta}
$$


where $\theta$ is real and positive [92]. Substituting equation (2.11) into the complex Hamiltonian, we have

$$
<\Psi_{t}|H(\theta)-W| \Psi_{t}>=\sum_{i} \sum_{j} C_{i} C_{j} H_{i j}-\sum_{i} \sum_{j} C_{i} C_{j} \delta_{i j} W=0,
$$

where $\mathrm{W}$ denotes energy. Using variational method, a variation of $\mathrm{W}$ with respect to $C_{i} \partial|W| / \partial C_{i}$ is carried out. This will lead to the usual eigenvalue equations

$$
\sum_{j}\left(H_{i j}-W^{\lambda} I\right) a_{j}^{\lambda}=0,
$$

with $W^{\lambda}$ and $a^{\lambda}$ the $\lambda$ th eigenvalues and eigenvectors, and I is the identity matrix. Under such a transformation, one calculates the kinetic and potential matrix elements separately, and scales according to the above scheme. Resonances can be examined once the complex eigenvalue problem is diagonalized. As in all variational calculations, a trial wave function is expanded in a linear combination of orthonormal basis function $\left|\chi_{i}\right\rangle$,

$$
\left|\Psi_{t}>=\sum_{i} C_{i}\right| \chi_{i}>
$$

and then the resulting complex eigenvalue problem solved. The advantage of using this method is that resonance parameters can be obtained by using bound-state-type wave function or $L^{2}$-type functions [92] and no asymptotically oscillating wave functions are necessary.

\subsection{Hyperspherical Method}

In this approach the key-point is in replacing the pair of radial distances $\left(\vec{r}_{1}, \vec{r}_{2}\right)$ of two electrons from the nucleus by the hyper-radius $\mathrm{R}$ which represent the scale of the system in 6 -dimension and by the mock angle $\alpha$ which represent the angular correlation $[30,74,81-83]$. The usual six variables 
$\left(r_{1}, \theta_{1}, \phi_{1}, r_{2}, \theta_{2}, \phi_{2}\right)$ for the two electrons are now replaced by $(R, \Omega)$, where $\Omega$ represents collectively the five angular coordinates $\left(\alpha, \theta_{1}, \phi_{1}, \theta_{2}, \phi_{2}\right)$. The eigenvalues are determined by using Born-Oppenheimer-type separation between $R$ and $\Omega$. In hyperspherical coordinates, the two electron Hamiltonian can be expressed as

$$
H=\frac{1}{2}\left[-R^{-5 / 2} \frac{\partial^{2}}{\partial R^{2}} R^{5 / 2}+\frac{\Lambda^{2}+15 / 4}{R^{2}}\right]+\frac{1}{R} C\left(\alpha, \theta_{12}\right) .
$$

The terms in the square bracket are, respectively, the radial and angular kinetic energy and the last term is the potential energy in (1.4). $\Lambda$ is called "grand angular momentum" because it involves all five variables $\Omega$. The joint influence of the centrifugal and Coulomb terms is in the eigenfunctions of the combined operator, the fixed R Hamiltonian

$$
H_{R}=\frac{1}{2}\left(\frac{\Lambda^{2}+15 / 4}{R^{2}}\right)+\frac{1}{R} C\left(\alpha, \theta_{12}\right),
$$

which depends on $\mathrm{R}$ parametrically. The system Hamiltonian therefore will be

$$
H=-\frac{1}{2} R^{-5 / 2} \frac{\partial^{2}}{\partial R^{2}} R^{5 / 2}+H_{R}
$$

Macek suggested that although two operators $\Lambda^{2}$ and $C(\Omega)$ do not commute, the total operator $H_{R}$ can nevertheless be diagonalized at each $\mathrm{R}$ such that the eigenvalues serves as potential-energy wells for electronic motion and the eigenvectors provide as adiabatic channel functions. Therefore, one solves this angular part of the problem

$$
H_{R} \Phi_{\mu}(R ; \Omega)=U_{\mu}(R) \Phi_{\mu}(R ; \Omega)
$$

to get adiabatic channel function $\Phi_{\mu}$ and eigenvalues $U_{\mu}(R)$ which converge to single ionization limit. Therefore, doubly excited states can be classified 
as different groups that correspond to the different thresholds of a parent ion (ion $+\mathrm{e}) . \Phi_{\mu}$ can be expanded in terms of a linear combination of nonorthgonal basis functions which contain both eigenfunctions of grand angular momentum operator and two-electron basis function representing the asymptotic channel function. With each adiabatic potential well, eigenvalues of the full Hamiltonian in (2.15) can be calculated by solving the Schrödinger equation numberically. In adiabatic treatment, the three body problems in collective coordinates at large $\mathrm{R}$ return to the independent particle electron coordinates for the asymptotic region.

\subsection{Our Method}

Our method of calculating doubly excited ridge states is most closely related to the adiabatic hyperspherical method [30,74,81-83]. In our method we retain the use of hyperspherical coordinates, however, we treat two electrons on par throughout. The potential wells converge to the double ionization limit unlike in the adiabatic approach in which the potential wells converge to single ionization limits. Our method contains four steps. The first step is diagonalization of the effective charge operator $\mathrm{C}$ in fixed $\lambda$ manifold. The second step is seeking an analytical expression for the lowest eigenvalue. The potential well in

each $\lambda$ becomes a simple and analytical expression $\left(U(R)=\frac{(\lambda+3 / 2)(\lambda+5 / 2)}{2 R^{2}}+\frac{C}{R}\right)$. The third step is to get from this $U(R)$ a set of eigenvalues described by a single the Rydberg formula. Each potential well supports an infinite number of eigenstates. We pick the lowest one in each of them to form a Rydberg formula. To improve our results, we go to the fourth step. In the fourth step we take off-diagonal coupling into account. For any given maximum $\lambda$ values, we diagonalize matrix elements $\left(U_{\lambda, \lambda^{\prime}}(R)=\frac{(\lambda+3 / 2)(\lambda+5 / 2)}{2 R^{2}} \delta_{\lambda, \lambda^{\prime}}+\frac{C_{\lambda, \lambda^{\prime}}}{R}\right)$ at each 
$R$. The eigenvalues provide the potential wells and the eigenvectors provide the basis for expansion of the full wavefunction. Expanding the full wavefunction in this basis and solving the Schrödinger equation numerically, we can get improved eigenvalues. The accuracy of the eigenvalues depend on the $\lambda_{\max }$. The larger the $\lambda_{\max }$ is the more accurate the eigenvalues are. 


\section{CHAPTER 3}

\section{HYPERSPHERICAL COORDINATES AND SCHRÖDINGER EQUATION}

\subsection{Hyperspherical Coordinates}

We begin by redefining the collective coordinates, called hyperspherical coordinates, which have been used very successfully for describing electron correlation and doubly excited states of atoms

$$
\begin{gathered}
R=\left(r_{1}^{2}+r_{2}^{2}\right)^{1 / 2} \\
\alpha=\tan ^{-1}\left(r_{2} / r_{1}\right) .
\end{gathered}
$$

Therefore, $\mathrm{R}$ represents the size of the system and $\alpha$ the relative radial excitation of the electrons. To describe the motion of two electrons in the field of a nucleus, we need 6 coordinates because there are 6 degrees of freedom. We can choose 3 Euler angles to describe the overall orientation of the system in space and three others to describe the internal degrees of freedom. We can use $r_{1}, r_{2}$ and $\theta_{12}$ as the internal set of three, and replace $r_{1}$ and $r_{2}$ by $\mathrm{R}$ and $\alpha$. $\theta_{12}$ is the angle between $\overrightarrow{r_{1}}$ and $\overrightarrow{r_{2}}$. See Fig. 5. Electron correlations are then described by two angles $\alpha$ and $\theta_{12}$, where $\alpha$ describes the radial correlation of the two electrons and $\theta_{12}$ describes the angular correlation of the two electrons. Usually, instead of using $\alpha, \theta_{12}$ and three Euler angles, it is more convenient

to use $\alpha, \hat{r}_{1}$ and $\hat{r}_{2}$ [where $\hat{r}_{i}=\left(\theta_{i}, \varphi_{i}\right)$ denotes the spherical angles of electron i] as five hyperspherical angles, and denote them collectively by $\Omega$. 
(a)

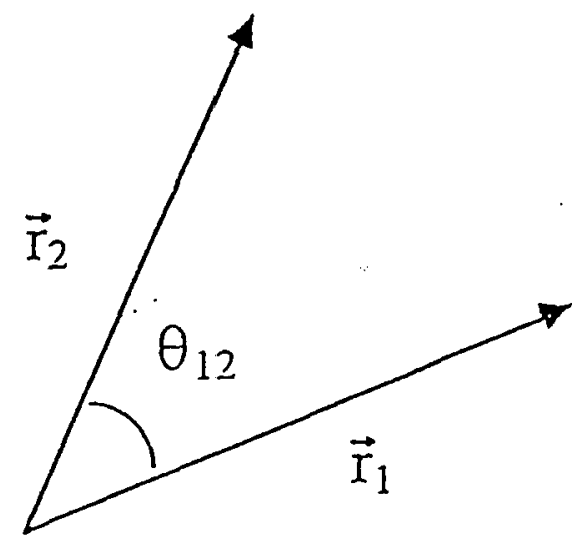

(b)

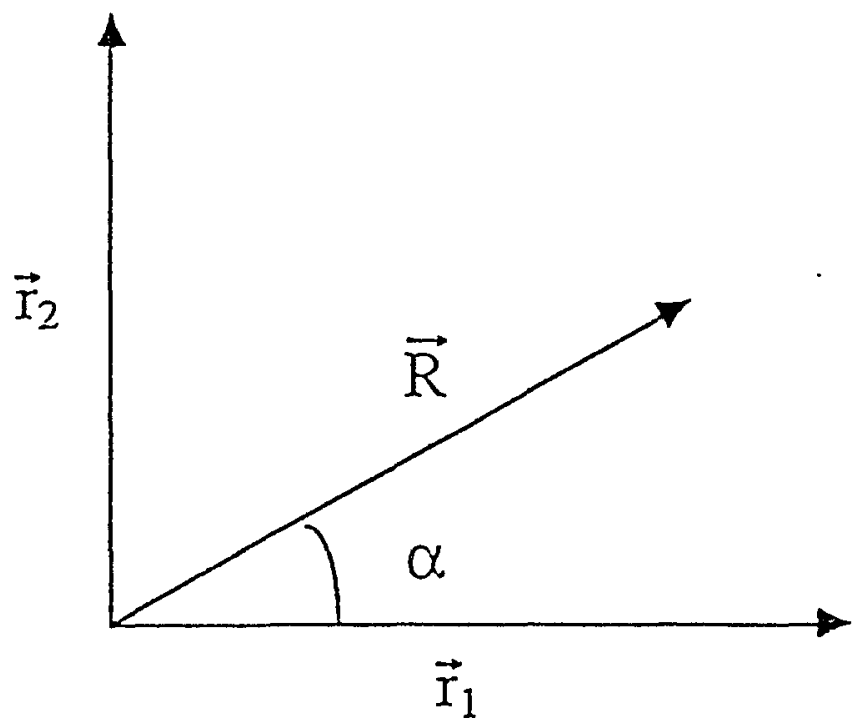

Figure 5. (a) Diagram of the two electron configuration. (b) Diagram of the relation between Cartesian and Hyperspherical coordinates. 


\subsection{Schrödinger Equation}

The two electron Hamiltonian in non-relativistic form is

$$
H=-\frac{1}{2} \nabla_{1}^{2}-\frac{1}{2} \nabla_{2}^{2}-\frac{Z}{r_{1}}-\frac{Z}{r_{2}}+\frac{1}{r_{12}},
$$

where we take the nucleus as having infinite mass, and use atomic units. The Schrödinger equation is

$$
H \Psi\left(\vec{r}_{1}, \vec{r}_{2}\right)=E \Psi\left(\vec{r}_{1}, \vec{r}_{2}\right)
$$

It can be written in hyperspherical coordinates as

$$
\left\{\frac{1}{2}\left[-R^{-5 / 2} \frac{\partial^{2}}{\partial R^{2}} R^{5 / 2}+\frac{\Lambda^{2}+15 / 4}{R^{2}}\right]+\frac{C\left(\alpha, \theta_{12}\right)}{R}\right\} \Psi\left(\vec{r}_{1}, \vec{r}_{2}\right)=E \Psi\left(\vec{r}_{1}, \vec{r}_{2}\right),
$$

where

$$
\Lambda^{2}=-\frac{1}{\sin ^{2} \alpha \cos ^{2} \alpha} \frac{d}{d \alpha}\left(\sin ^{2} \alpha \cos ^{2} \alpha \frac{d}{d \alpha}\right)+\frac{\mathbf{l}_{1}^{2}}{\cos ^{2} \alpha}+\frac{\mathbf{l}_{2}^{2}}{\sin ^{2} \alpha}
$$

is the square of the grand angular momentum operator $\left(\mathbf{l}_{1}\right.$ and $\mathbf{l}_{2}$ are the usual one-electron angular momentum operators) and

$$
C\left(\alpha, \theta_{12}\right)=-\frac{Z}{\cos \alpha}-\frac{Z}{\sin \alpha}+\frac{1}{\left(1-\sin 2 \alpha \cos \theta_{12}\right)^{1 / 2}}
$$

is the effective charge. In equation (3.6), $\mathrm{Z}$ is the charge of the nucleus. The effective charge $\mathrm{C}$ includes both the electron-electron and electron-nucleus interactions. In Fig. 6 , we display the effective charge $\mathrm{C}$ on the $\left(\alpha, \theta_{12}\right)$ plane for $\mathrm{Z}=1$. Fig. 6 shows a flat saddle at $\alpha=45^{\circ}$ and $\theta_{12}=180^{\circ}$, and deep valleys at $\alpha=0^{\circ}$ and $90^{\circ}$. In the region $r_{1}=r_{2}$, which corresponds to $\alpha=45^{\circ}$, the potential energy still depends on $\theta_{12}$. When $\theta_{12}=0$ and $\alpha=45^{\circ}$, the two electrons are nearly on top of each other where the electron-electron repulsion causes the sharp spike as shown in Fig. 6. When $\theta_{12}=180^{\circ}$ and $\alpha=45^{\circ}$, 


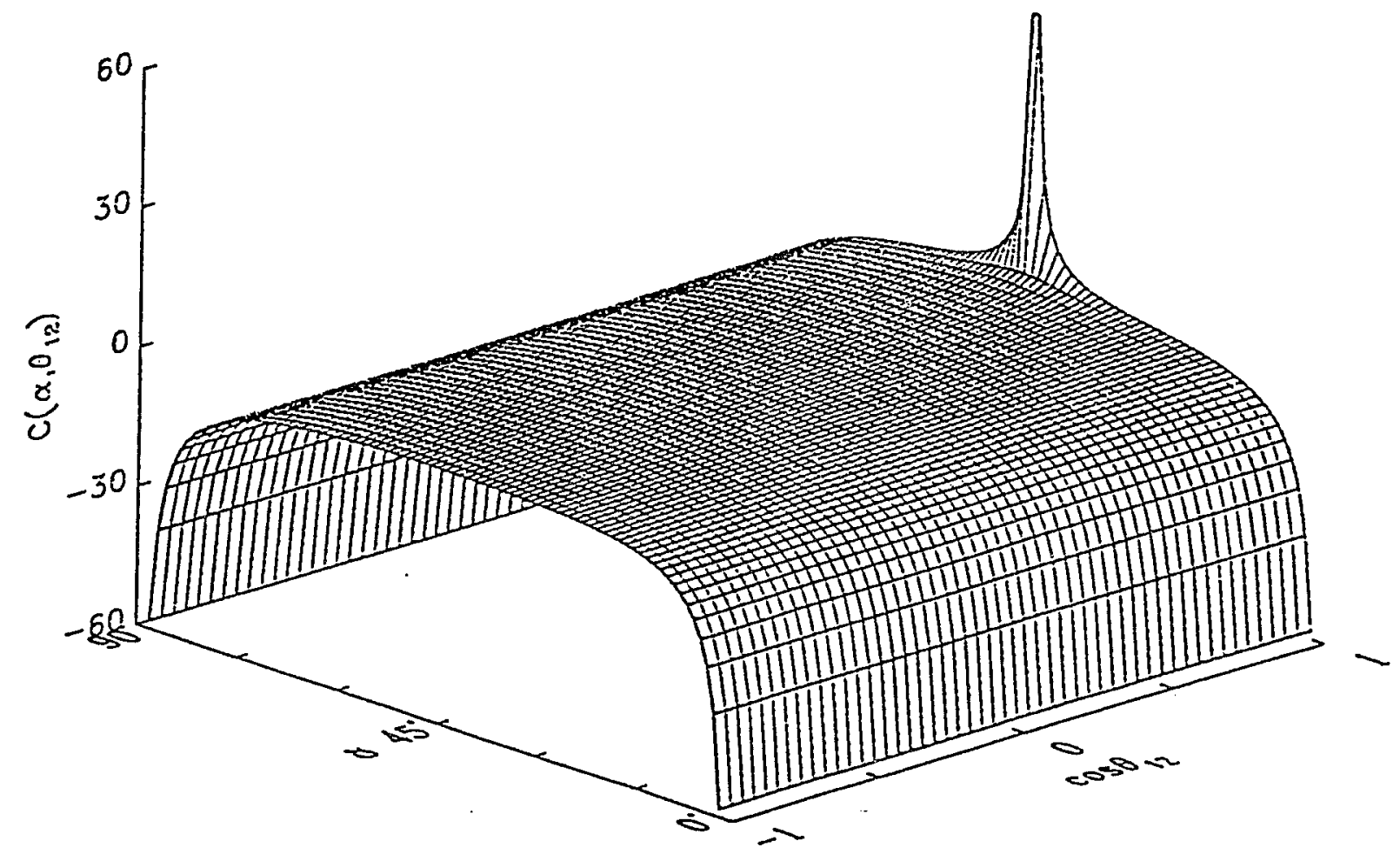

Figure 6. Potential function $\mathrm{C}\left(\alpha, \theta_{12}\right)$ in atomic units for a pair of electrons in the field of a proton. 
the potential is unstable for departures in $\alpha=45^{\circ}$, while it is stable for departure along the coordinate $\theta_{12}$. This structure of the surface has an essential influence on electron correlations.

The eigenvalues and eigenfunctions of the squared grand angular momentum $\Lambda^{2}$ are known analytically as they are for the orbital momentum $l^{2}$. The eigenvalues are represented by

$$
\Lambda^{2}=\lambda(\lambda+4), \quad \lambda=0,1,2, \ldots
$$

and are degenerate for $\lambda \neq 0$. It is also convenient to introduce a related quantum number $n_{r c}$ that indexes the number of nodes in $\alpha$,

$$
\lambda=l_{1}+l_{2}+2 n_{r c}
$$

The eigenfunctions $\Phi_{n_{r c} l_{1} l_{2} L M}^{S \pi}(\Omega)$, with symmetry $(-1)^{S}$ for singlet $(\mathrm{S}=0)$ or triplet $(\mathrm{S}=1)$ states with fixed $L, M, S, \pi$ are given by

$$
\begin{aligned}
\Phi_{n_{r c} l_{1} l_{2} L M}^{S \pi}(\Omega)= & \frac{1}{\sqrt{2}}\left[\phi_{n_{r c} l_{1} l_{2} L M}\left(\alpha, \hat{r}_{1}, \hat{r}_{2}\right)+(-)^{l_{1}+l_{2}-L+S+n_{r c}} \phi_{n_{r c} l_{2} l_{1} L M}\left(\alpha, \hat{r}_{1}, \hat{r}_{2}\right)\right] \\
& \text { if } \quad l_{1} \neq l_{2} \\
= & \frac{1}{2}\left[1+(-1)^{-L+S+n_{r c}}\right] \phi_{n_{r c} l l L M}\left(\alpha, \hat{r}_{1}, \hat{r}_{2}\right) \\
& \text { if } \quad l_{1}=l_{2}=l .
\end{aligned}
$$

The function $\phi_{n_{r c} l_{1} l_{2} L M}$ can be expressed as

$$
\begin{aligned}
\phi_{n_{r c} l_{1} l_{2} L M}\left(\alpha, \hat{r}_{1}, \hat{r}_{2}\right)= & N_{n_{r c} l_{1} l_{2}}\left(\cos ^{l_{1}} \alpha\right)\left(\sin ^{l_{2}} \alpha\right) \times Y_{l_{1} l_{2} L M}\left(\hat{r}_{1}, \hat{r}_{2}\right) \\
& \times_{2} F_{1}\left(-n_{r c}, n_{r c}+l_{1}+l_{2}+2, l_{2}+\frac{3}{2} ; \sin ^{2} \alpha\right),
\end{aligned}
$$

where $Y_{l_{1} l_{2} L M}$ is a standard "coupled harmonic" as given in Eq. (10.5) of Ref. 39

$$
Y_{l_{1} l_{2} L M}\left(\hat{r}_{1}, \hat{r}_{2}\right)=\sum_{m_{1} m_{2}}\left(l_{1} l_{2} L M \mid l_{1} m_{1}, l_{2} m_{2}\right) Y_{l_{1} m_{1}}\left(\hat{r}_{1}\right) Y_{l_{2} m_{2}}\left(\hat{r}_{2}\right)
$$


$Y_{l_{1} l_{2} L M}$ is the total orbital angular-momentum eigenfunction of the pair. $N_{n_{r c} l_{1} l_{2}}$ is a normalization constant and given by

$$
\begin{aligned}
N_{n_{r c} l_{1} l_{2}}= & 2^{l_{1}+l_{2}+1} \frac{l_{2} !}{\left(2 l_{2}+1\right) !}\left[\frac{\left(2 n_{r c}+2 l_{2}+1\right) !\left(n_{r c}+l_{1}\right) !}{\left(n_{r c}+l_{2}\right) !\left(2 n_{r c}+2 l_{1}+1\right) !}\right]^{1 / 2} \\
& \times\left[\frac{2\left(2 n_{r c}+l_{1}+l_{2}+2\right)\left(n_{r c}+l_{1}+l_{2}+1\right) !}{n_{r c} ! \pi}\right]^{1 / 2} .
\end{aligned}
$$

In (3.10) ${ }_{2} F_{1}\left(-n_{r c}, n_{r c}+l_{1}+l_{2}+2, l_{2}+\frac{3}{2} ; \sin ^{2} \alpha\right)$ is proportional to a Jacobi polynomial of degree $n_{r c}$ in $\sin ^{2} \alpha$, with $n_{r c}$ nodes in the range $0<\alpha<\frac{1}{2} \pi$. Since we are always interested in the states with fixed S, L, M and $\pi$, these indices will usually be dropped. $Y_{l_{1} l_{2} n_{\mathrm{rc}}}\left(\hat{r}_{1}, \hat{r}_{2}\right)$ has a simple analytical form if $\mathrm{L}=0$ and $l_{1}=l_{2}=l$

$$
Y_{l n_{r c}}\left(\hat{r}_{1}, \hat{r}_{2}\right)=(-1)^{l} \sqrt{\frac{2 l+1}{4 \pi}} P_{l}\left(\cos \theta_{12}\right),
$$

where $P_{l}\left(\cos \theta_{12}\right)$ is the Legendre polynomial.

\section{$3.3 \quad 3-j$ and 6-j Symbols}

Here we are going to introduce some formulae for $3-j$ and $6-j$ symbols [93-94] which are involved in the coupling of two electrons and are used in this thesis.

The Wigner 3-j symbol

$$
\left(\begin{array}{ccc}
j_{1} & j_{2} & j_{3} \\
m_{1} & m_{2} & m_{3}
\end{array}\right),
$$

is related to the Clebsch-Gordan coupling coefficient in (3.11) and is nonvanishing if the following two conditions are fulfilled

$$
\begin{gathered}
m_{1}+m_{2}=-m_{3}, \\
j_{3}=j_{1}+j_{2}, j_{1}+j_{2}-1, \ldots,\left|j_{1}-j_{2}\right| .
\end{gathered}
$$


In particular, when $m_{1}=m_{2}=m_{3}=0$, the $3-\mathrm{j}$ symbols have a simple analytical form

$$
\left(\begin{array}{ccc}
j_{1} & j_{2} & j_{3} \\
0 & 0 & 0
\end{array}\right)=(-1)^{g} \sqrt{\frac{\left(2 g-2 j_{1}\right) !\left(2 g-2 j_{2}\right) !\left(2 g-2 j_{3}\right) !}{(2 g+1) !}} \frac{g !}{\left(g-j_{1}\right) !\left(g-j_{2}\right) !\left(g-j_{3}\right) !},
$$

if $j_{1}+j_{2}+j_{3}=2 g$, where $g$ is an integer, and

$$
\left(\begin{array}{ccc}
j_{1} & j_{2} & j_{3} \\
0 & 0 & 0
\end{array}\right)=0
$$

if $j_{1}+j_{2}+j_{3}=2 g+1$.

The Wigner 6-j symbol is

$$
\left\{\begin{array}{lll}
j_{1} & j_{2} & j_{3} \\
l_{1} & l_{2} & l_{3}
\end{array}\right\}
$$

and it is non-vanishing if the following triangular conditions are fulfilled $\Delta\left(j_{1} j_{2} j_{3}\right)$, $\Delta\left(l_{2} l_{3} j_{3}\right), \Delta\left(j_{1} l_{2} l_{3}\right)$ and $\Delta\left(j_{2} l_{1} l_{3}\right)$, these triplets forming legs of closed triangles. Here, we list the useful formulae

$$
\begin{gathered}
\begin{array}{c}
\left\{\begin{array}{ccc}
a & b & c \\
1 & c-1 & b+1
\end{array}\right\}=(-1)^{s}\left[\frac{(s-2 b)(s-2 b-1)(s-2 c+1)(s-2 c+2)}{(2 b+1)(2 b+2)(2 b+3)(2 c-1) 2 c(2 c+1)}\right]^{1 / 2} \\
\left\{\begin{array}{ccc}
a & b & c \\
1 & c & b
\end{array}\right\}=(-1)^{s} \frac{2 X}{[2 b(2 b+1)(2 b+2) 2 c(2 c+1)(2 c+2)]^{1 / 2}} \\
\left\{\begin{array}{ccc}
a & b & c \\
2 & c-1 & b+1
\end{array}\right\}=(-1)^{s} \frac{4[(a+b+2)(a-b-1)-(c-1)(b+c+2)]}{[2 b(2 b+1)(2 b+2)(2 b+3)(2 b+4)]^{1 / 2}} \\
\times \frac{[(s-2 b-1)(s-2 b)(s-2 c+1)(s-2 c+2)]^{1 / 2}}{[(2 c-2)(2 c-1) 2 c(2 c+1)(2 c+2)]^{1 / 2}}
\end{array}
\end{gathered}
$$




$$
\begin{aligned}
\left\{\begin{array}{ccc}
a & b & c \\
2 & c & b
\end{array}\right\}= & (-1)^{s} \frac{2[3 X(X+1)-4 b(b+1) c(c+1)]}{[(2 b-1) 2 b(2 b+1)(2 b+2)(2 b+3)]^{1 / 2}} \\
& \times \frac{1}{[(2 c-1) 2 c(2 c+1)(2 c+2)(2 c+3)]^{1 / 2}}
\end{aligned}
$$

where $s=a+b+c$ and $X=a(a+1)-b(b+1)-c(c+1)$. 


\section{CHAPTER 4}

\section{ELECTRON-PAIR ANALYSIS FOR DOUBLY EXCITED RIDGE STATES I: $\mathbf{L}=0$}

\subsection{Introduction}

For the very high doubly excited ridge states that are of interest to us, we keep the collective, pair description throughout, including when $R \longrightarrow \infty$. No reference to individual electrons is made, no single ionization thresholds or single electron quantum numbers invoked. The only relevant ionization limit is the one of double ionization when the "grand parental ion" (in our case, the bare nucleus) and the pair of electrons mutually separate to infinity $(R \longrightarrow \infty)$. In the adiabatic-hyperspherical method, it has been recognized that the successive potential wells in $\mathrm{R}$ that converge to successive single ionization thresholds show avoided crossings with each other. An important sequence of crossings has a locus in $\mathrm{R}$ which tracks the ridge line of the potential in (1.4). Couplings between potential curves along this locus has been recognized as providing an "excitation ladder" to high excitation [42]. A diabatic tracing of the potential along this locus would also provide a single potential well that converges finally to the double ionization limit. Such a procedure of arriving at this well through first calculating adiabatic potential wells and then including non-adiabatic couplings between them seems, however, hopelessly cumbersome and impractical, particularly when we are interested in high lying states. As with all Coulomb problems, the various thresholds $\mathrm{N}$ pile up closer and closer together and the 
number of adiabatic potentials and couplings grows explosively large. A more direct approach to calculating potentials that converges to the double ionization limit is desirable and this is what we address in this chapter.

\subsection{Pair Analysis of Two Electron States}

The different scaling in $\mathrm{R}$ of the angular kinetic energy and the potential in (3.4) makes the two-electron equation non-separable in hyperspherical coordinates just as it is in independent particle coordinates. The adiabatichyperspherical method proceeds by seeking eigenstates of $\Lambda^{2}+\mathrm{RC}$ in (3.4) at each R. Typically, a basis of products of one-electron functions is employed, and to speed convergence at large $\mathrm{R}$ this product is chosen so as to converge to single-ionization thresholds. Instead, we proceed as follows. Each $\lambda$ manifold (except for $\lambda=0$ ) is degenerate and this degeneracy increases with $\lambda$. We diagonalize $C\left(\alpha, \theta_{12}\right)$ within each $\lambda$ manifold. The eigenvectors are simultaneously eigenstates of $\Lambda^{2}$ and $C\left(\alpha, \theta_{12}\right)$. They provide at each $\mathrm{R}$ a basis for expansion of the full wave function $\Psi(R, \Omega)$, with radial functions $\vec{F}(R)$ as the expansion coefficients. The eigenvalues of $\Lambda^{2}$ and $C\left(\alpha, \theta_{12}\right)$ provide diagonal potential terms (wells) in the resulting radial equations for $\vec{F}(R)$. These potential wells converge to $\mathrm{E}=0$ as $R \longrightarrow \infty$, that is, to the double ionization limit.

In this chapter, we restrict ourselves to $\mathrm{L}=0$. Although algebraically more involved, the extension to other $\mathrm{L}$ values is straightforward and we will return to it in the next chapter. The $\mathrm{L}=0$ case has the merit of making our procedure and the form of its results more transparent. In this case, we have $l_{1}=l_{2}=l$, and $\Omega$ reduces to just the two angular pair coordinates, $\alpha$ and $\theta_{12}$, with Y becoming

$$
Y_{l l L=M=0}=(-)^{l}\left[\frac{2 l+1}{4 \pi}\right]^{\frac{1}{2}} P_{l}\left(\cos \theta_{12}\right) .
$$


The functions $\Phi$ and $\phi$ become identical, and in $\lambda=2\left(l+n_{r c}\right), n_{r c}$ takes even(odd) values for $\mathrm{S}=0(1)$; correspondingly $\lambda / 2$ is even(odd) for $l+S$ even(odd). The normalized eigenfunctions of $\Lambda^{2}$ for $L=0$ are, therefore,

$$
\begin{gathered}
\Phi_{n_{r c l} l}\left(\alpha, \theta_{12}\right)=(-)^{l} N_{n_{r c l} l} \sin ^{l} \alpha \cos ^{l} \alpha P_{n_{r c}}^{\left(l+\frac{1}{2}, l+\frac{1}{2}\right)}(\cos 2 \alpha) P_{l}\left(\cos \theta_{12}\right) \\
N_{n_{r c l} l}=2^{2\left(n_{r c}+l\right)+1} \frac{\left(n_{r c}+l\right) !}{\left(2 n_{r c}+2 l+1\right) !}\left[\frac{2(2 l+1) n_{r c} !\left(n_{r c}+2 l+1\right) !\left(n_{r c}+l+1\right)}{\pi}\right]^{1 / 2}
\end{gathered}
$$

where $P_{n}^{(\alpha, \beta)}$ is a Jacobi Polynomial (equivalently, a Gegenbauer or Ultraspherical Polynomial [95]) and $P_{l}$ is a Legendre Polynomial.

4.2.1 Diagonalization at Fixed $\lambda$ With the eigenfunctions in (4.2) for a degenerate manifold of $\lambda=2\left(l+n_{r c}\right)$, we diagonalize the effective charge operator $C\left(\alpha, \theta_{12}\right)$. The required matrix elements are

$$
\begin{aligned}
\left.<n_{r c} l\left|C\left(\alpha, \theta_{12}\right)\right| n_{r c}^{\prime} l^{\prime}\right\rangle= & \int_{0}^{\frac{\pi}{2}} \sin ^{2} \alpha \cos ^{2} \alpha d \alpha \\
& \times \int_{0}^{\pi} \sin \theta_{12} d \theta_{12} \Phi_{n_{r c} l} \Phi_{n_{r c}^{\prime} l^{\prime}} C\left(\alpha, \theta_{12}\right) .
\end{aligned}
$$

The matrix elements can be calculated numerically. The eigenvalues $C_{\lambda Q}$ of this matrix are tabulated for ${ }^{1} S$ and ${ }^{3} S$ states of He ( $\mathrm{Z}=2$ in (3.6)) in Tables 4.1 and 4.2 , respectively, for $\lambda \leq 20$, and a representative sample of ${ }^{1} S$ eigenvalues are plotted in Fig. 7 for $\mathrm{H}^{-}(\mathrm{Z}=1)$. In each of these presentations, the eigenvalues divide into two groups as $\lambda$ runs through alternate even integers and, correspondingly, $l+S$ is alternately even and odd. The eigenvalues are labeled by $Q=0,1,2, . . ;$ the maximum value of $\mathrm{Q}$ is $(\lambda-2) / 4$ for odd values of $\lambda / 2$ whereas it is $\lambda / 4$ for ${ }^{1} S$ and $(\lambda-4) / 4$ for ${ }^{3} S$ for even values of $\lambda / 2$.

The eigenvalues have been plotted in Fig. 7 as continuous curves although Q only takes discrete integer values, in order to show the shape of 
Table 4.1. Eigenvalues of effective charge operator $C_{\lambda Q}$ for ${ }^{1} S$ states of He with $\lambda \leq 20$.

\begin{tabular}{lllllll}
$\lambda \backslash \mathrm{Q}$ & 0 & 1 & 2 & 3 & 4 & 5 \\
\hline 0 & -5.5902 & & & & & \\
2 & -4.6995 & & & & & \\
4 & -4.3895 & -8.44130 & & & & \\
6 & -4.2075 & -6.55930 & & & & \\
8 & -4.0823 & -5.86720 & -9.7461 & & & \\
10 & -3.9856 & -5.4866 & -7.5756 & & & \\
12 & -3.9077 & -5.2413 & -6.7084 & -10.6039 & & \\
14 & -3.8416 & -5.0663 & -6.2114 & -8.2981 & & \\
16 & -3.7848 & -4.9339 & -5.8832 & -7.3343 & -11.2443 & \\
18 & -3.7344 & -4.8288 & -5.6466 & -6.7657 & -8.8603 & \\
20 & -3.6894 & -4.7427 & -5.4667 & -6.3825 & -7.8355 & -11.7555
\end{tabular}

Table 4.2. Eigenvalues of effective charge operator $C_{\lambda Q}$ for ${ }^{3} S$ states of He with $\lambda \leq 20$.

\begin{tabular}{llllll}
$\lambda \backslash \mathrm{Q}$ & 0 & 1 & 2 & 3 & 4 \\
\hline 2 & -7.4392 & & & & \\
4 & -5.8854 & & & & \\
6 & -5.3460 & -9.1863 & & & \\
8 & -5.0529 & -7.1350 & & & \\
10 & -4.8632 & -6.3421 & -10.2152 & & \\
12 & -4.7264 & -5.8958 & -7.9703 & & \\
14 & -4.6212 & -5.6040 & -7.0499 & -10.9465 & \\
16 & -4.5364 & -5.3949 & -6.5138 & -8.5992 & \\
18 & -4.4658 & -5.2363 & -6.1556 & -7.6028 & -11.5142 \\
20 & -4.4054 & -5.1107 & -5.8956 & -7.00845 & -9.1039
\end{tabular}




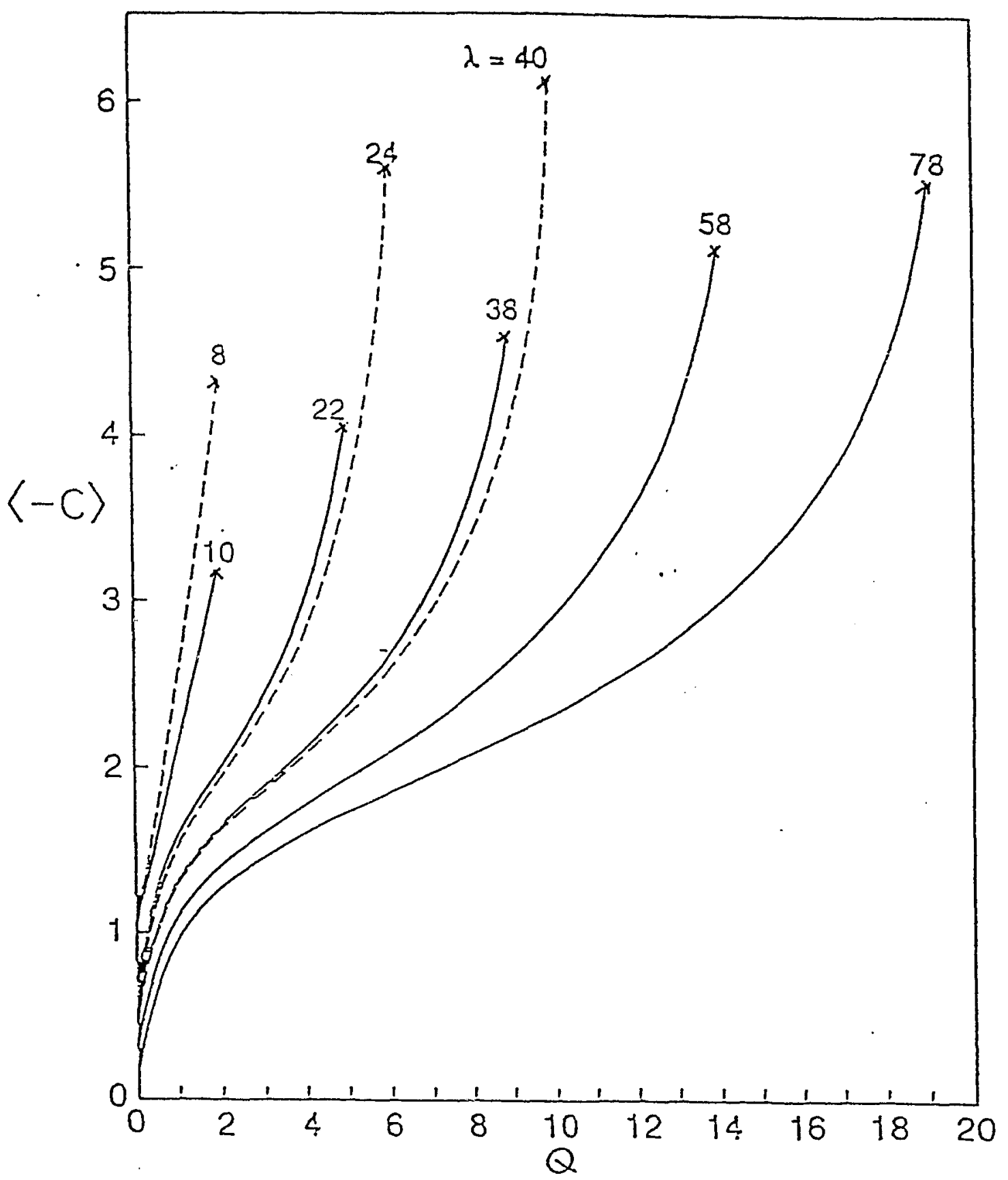

Figure 7. Results of numerical diagonalization of the potential in (4.4) are shown as continuous curves, dashed(solid) for $\lambda / 2$ even(odd). Crosses give maximum eigenvalues as given by the approximate, analytical expressions in (4.6) and (4.9). 


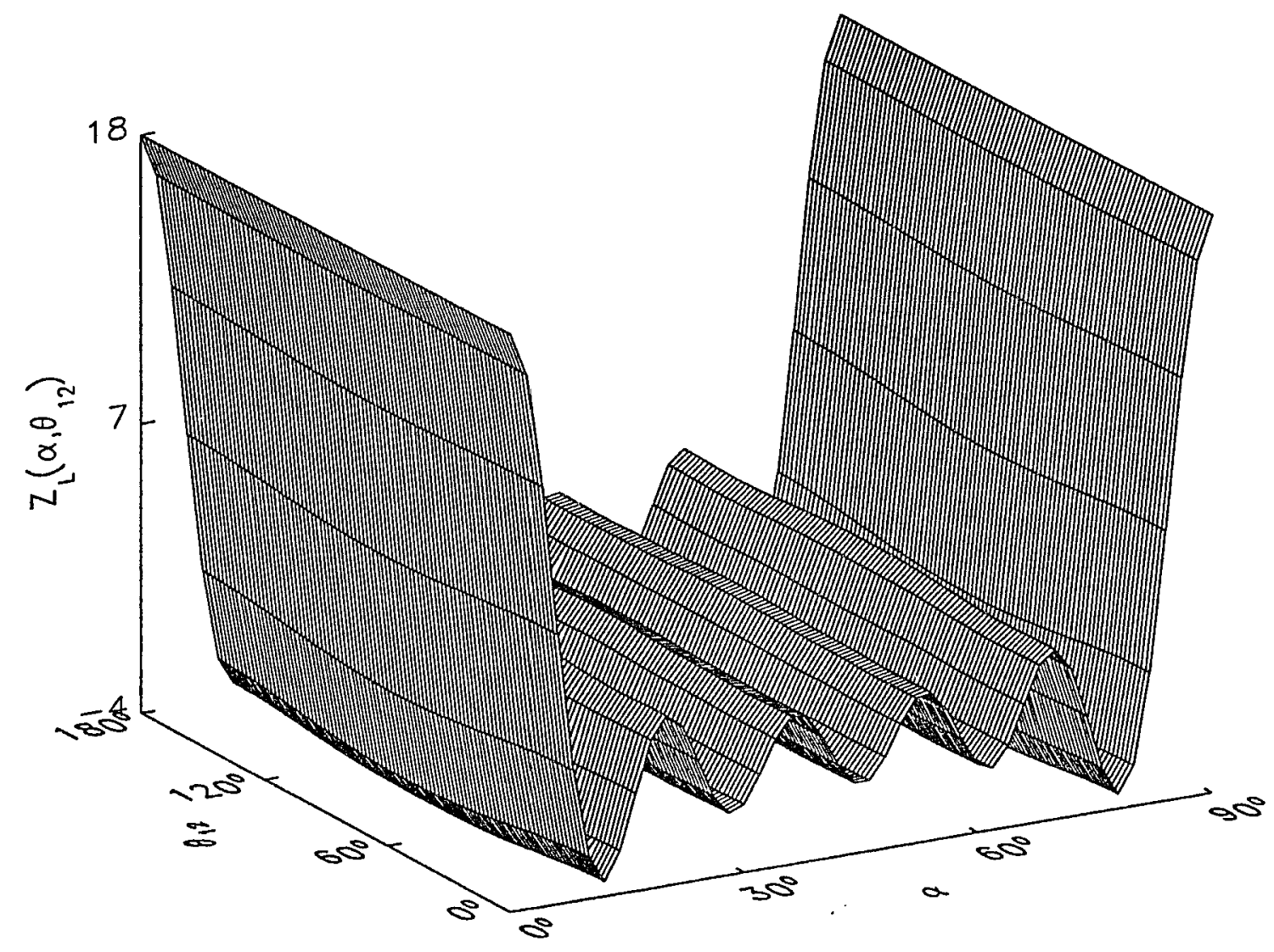

Figure 8: The lowest eigenvector distribution in $\left(\alpha, \theta_{12}\right)$ space with $\lambda=20$. 


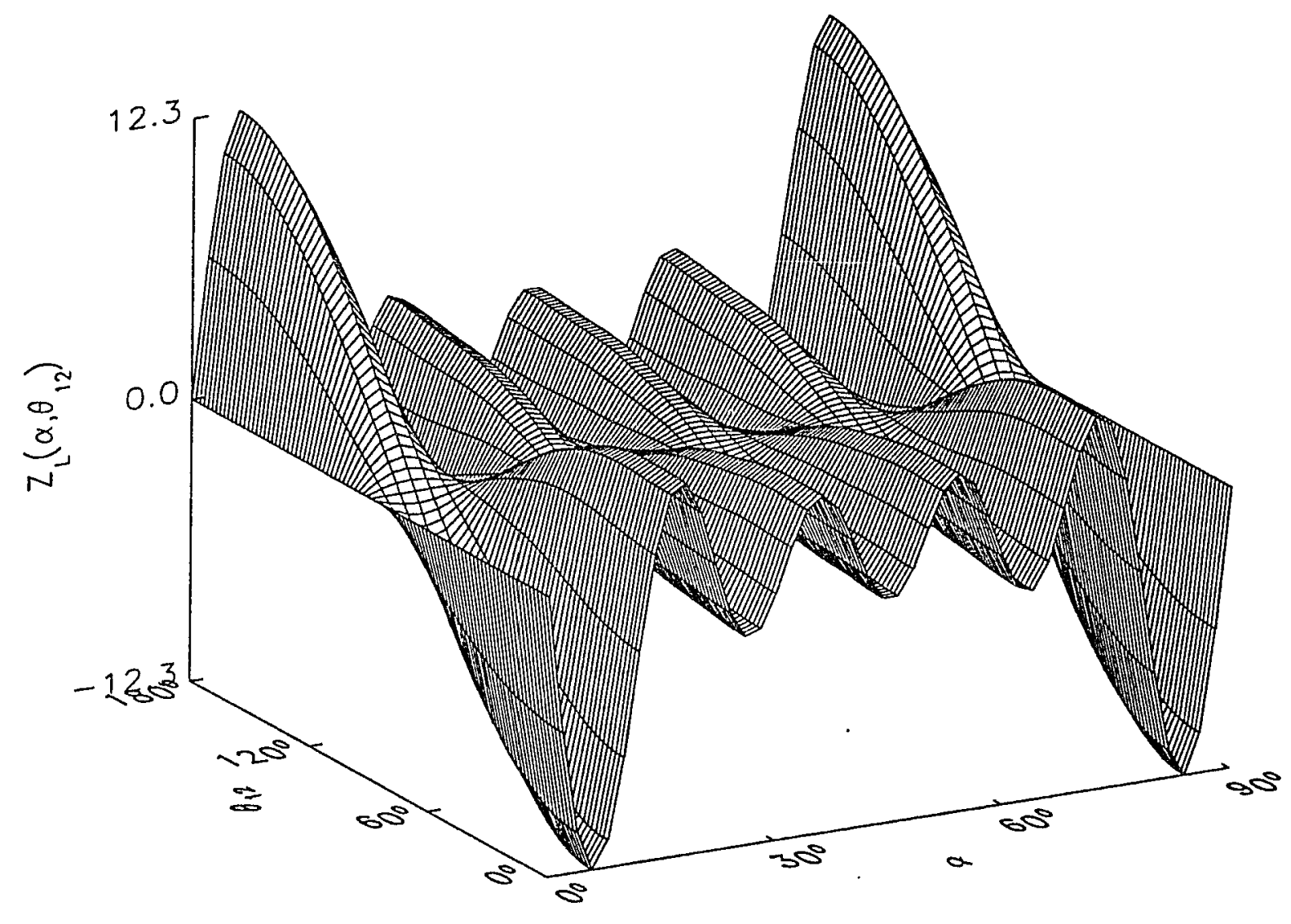

Figure 9: The lowest eigenvector distribution in $\left(\alpha, \theta_{12}\right)$ space with $\lambda=18$. 


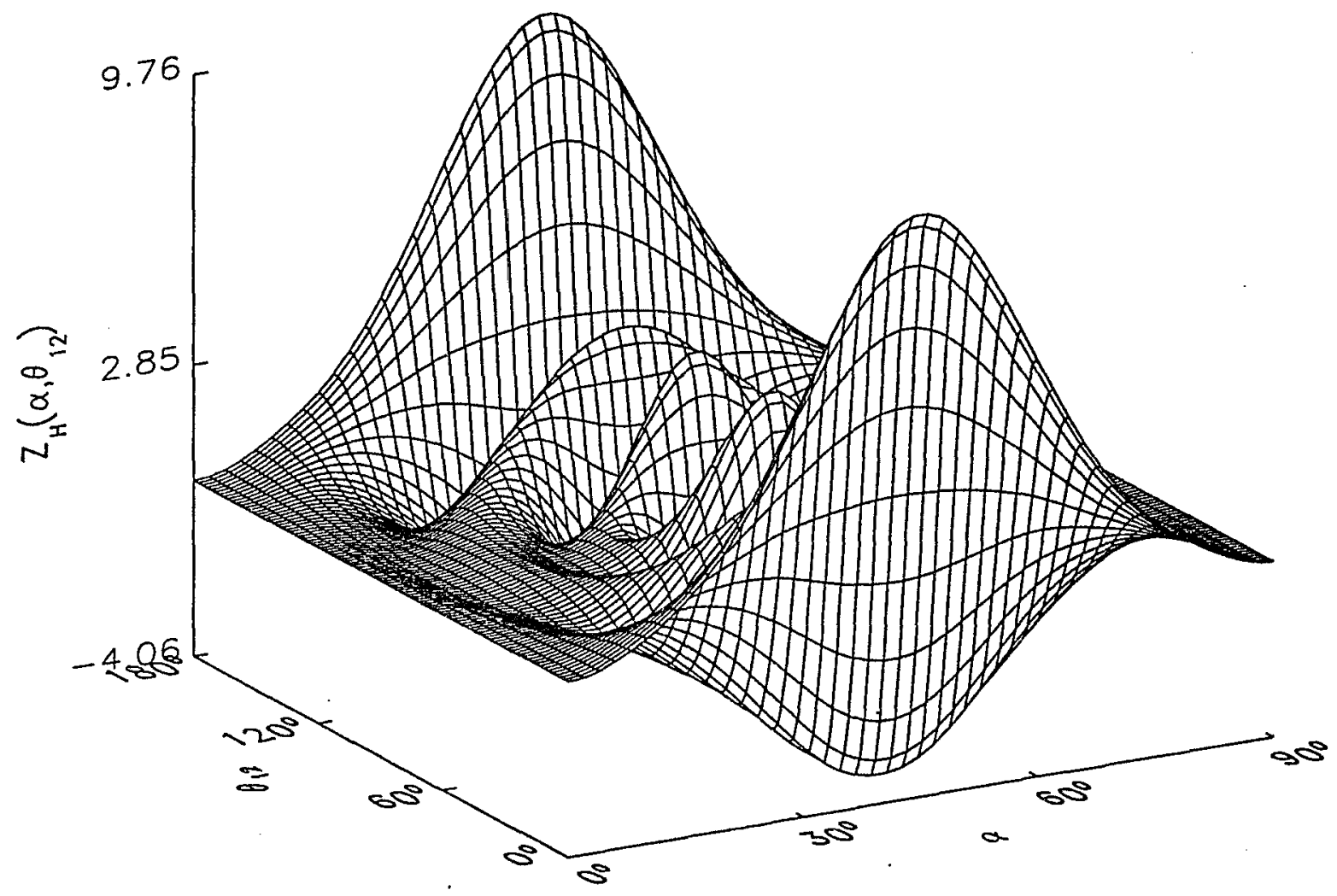

Figure 10: The highest eigenvector distribution in $\left(\alpha, \theta_{12}\right)$ with $\lambda=20$. 


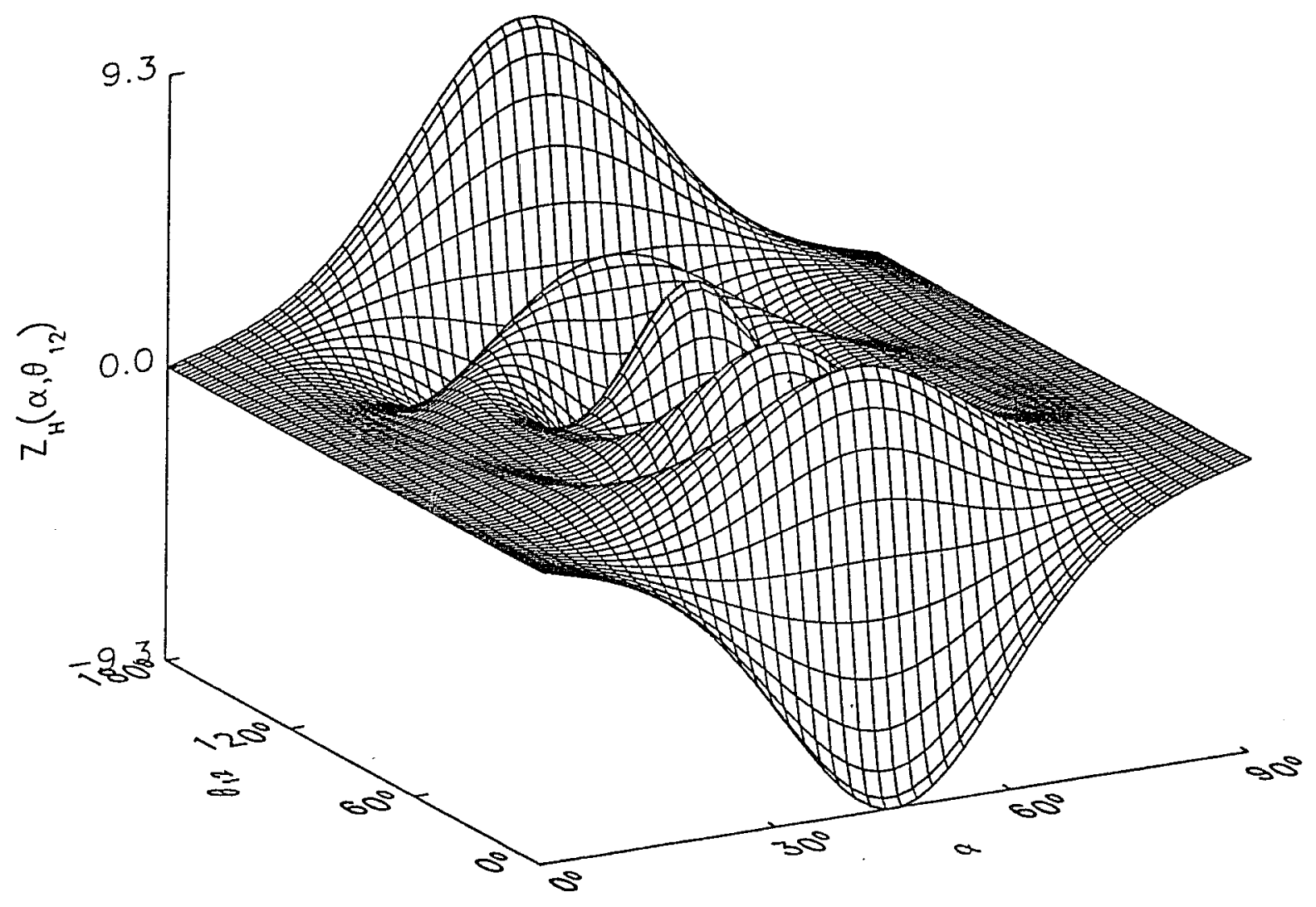

Figure 11: The highest eigenvector distribution in $\left(\alpha, \theta_{12}\right)$ with $\lambda=18$. 
their distribution at each $\lambda$. In particular, these plots are strikingly similar to other diagonalizations when an external perturbation mixes states of degenerate atomic manifolds (Appendix A) $[84,96]$. As in those examples, the extreme eigenvectors have special features and this is shown in Figs. $8,9,10$ and 11 which plot their distribution in $\left(\alpha, \theta_{12}\right)$ space. The lowest eigenvector of $C\left(\alpha, \theta_{12}\right)$, that is, the deepest potential providing therefore strongest binding, shows (Figs. 8 and 9) a concentration around $\alpha \simeq \pi / 2$ and 0 . This eigenvector is substantially independent of $\theta_{12}$ for even $\lambda / 2$ (Fig. 8), whereas for odd $\lambda / 2$ (Fig. 9) it shows a concentration around $\theta_{12} \simeq 0$ and $\pi$ and $\alpha \simeq 0$ and $\pi / 2$. On the other hand, the highest eigenvector of $C\left(\alpha, \theta_{12}\right)$ exhibits (Fig. 10 and 11) a concentration around $\alpha \simeq \pi / 4$ and $\theta_{12} \simeq \pi$ and 0 , the distribution of the eigenvector being symmetric around $\theta_{12} \simeq \pi / 2$ for even $\lambda / 2$ and antisymmetric for odd $\lambda / 2$.

\subsubsection{Analytical Results for the Extreme Eigenvalues Sim-} ple but quite accurate analytical expressions for the extreme eigenvalues follow upon observing that the matrix elements of $\mathrm{C}$ in (4.4) are dominated by the diagonal terms. Table 4.3 documents this for a representative $\lambda$. It follows that the extreme eigenvalues are well approximated by the diagonal matrix elements in (4.4) with highest and lowest values of $l$ (correspondingly, the lowest and highest values of $n_{r c}$ ). Indeed, the symmetries noted in the previous paragraph and in Figs. 8, 9, 10 and 11 of the extreme eigenvectors reflect the symmetries of the function $\Phi$ in (4.2) with highest and lowest allowed $l$. In either of these cases, $\Phi$ in (4.2) takes a simple form and the matrix element can be evaluated analytically. Since it is of less interest, we do not record here 
Table 4.3: Matrix elements of $C\left(\alpha, \theta_{12}\right)$ in $\lambda=12$ subspace.

$\begin{array}{lllll} & \mid 600> & \mid 422> & \mid 244> & \mid 066> \\ \mid 600> & -10.5964 & -0.1575 & 0.7466 & -0.5910 \\ \mid 422> & -0.1575 & -6.6764 & -0.2239 & 0.1488 \\ \mid 244> & 0.7466 & -0.2239 & -5.1992 & -0.2915 \\ \mid 066> & -0.5910 & 0.1489 & -0.2915 & -3.9892\end{array}$

the largest eigenvalue (least $n_{r c}$ ) which corresponds to least attraction. But the most attractive potentials can be obtained as follows.

Considering ${ }^{1} S$ symmetry, when $\lambda / 2$ is even, the lowest eigenvalue of $\mathrm{C}$ is approximated by the matrix element in (4.4) with $l=0, n_{r c}=\lambda / 2$. The wave function in (4.2) reduces to

$$
\Phi=\sqrt{\frac{8}{\pi}} \frac{\sin (\lambda+2) \alpha}{\sin 2 \alpha}
$$

This gives

$$
C_{\lambda Q=\lambda / 4} \simeq<\frac{1}{2} \lambda, 0|C| \frac{1}{2} \lambda, 0>=-\frac{8 Z}{\pi} \sum_{k=1}^{\lambda+2} \frac{1}{2 k-1}+\frac{4 \sqrt{2}}{\pi} \sum_{k=1}^{\lambda+2} \frac{(-)^{[k / 2]}}{2 k-1},
$$

where $[k / 2]$ is the integer part of $k / 2$. This value in (4.6), which can be thought of as an effective charge, can be simplified further, particularly for large $\lambda$

$$
C_{\lambda \lambda / 4} \simeq-\left(\frac{4}{\pi}\right)[Z(\gamma+\ln (4 \lambda+10))-\sqrt{2}(0.62)],
$$

where $\gamma=0.57721$ is Euler's constant.

For an odd $\lambda / 2$, the lowest eigenvalue of $\mathrm{C}$ is approximated by the matrix elements in (4.4) with $l=1, n_{r c}=(\lambda-2) / 2$. The wavefunction in (4.2) reduces to

$$
\Phi=-A \frac{\cos \theta_{12}}{\sin ^{2} 2 \alpha}\left[\left(n_{r c}+3\right) \sin 2\left(n_{r c}+1\right) \alpha-\left(n_{r c}+1\right) \sin 2\left(n_{r c}+3\right) \alpha\right],
$$


where $A=\sqrt{6} /\left[\left(n_{r c}+3\right)\left(n_{r c}+1\right) \pi\right]^{1 / 2}$. We obtain a similar but somewhat more complicated expression than (4.6) involving analytical sums for

$$
\begin{aligned}
& C_{\lambda(\lambda-2) / 4} \simeq<\frac{1}{2} \lambda-1,1\left|C\left(\alpha, \theta_{12}\right)\right| \frac{1}{2} \lambda-1,1>=-A^{2} \frac{Z}{3}\left(n_{r c}+1\right)^{2}\left(n_{r c}+3\right)^{2} \\
& \times\left[2 \sum_{j=1}^{n_{r c}+2} \sum_{k=1}^{n_{r c}} \sum_{l=1}^{k+j} b\left(n_{r c}+3, j\right) b\left(n_{r c}+1, k\right) \frac{1}{(2 l-1)}\right. \\
& -\sum_{j=1}^{n_{r c}} \sum_{k=1}^{n_{r c}} \sum_{l=1}^{k+j} b\left(n_{r c}+1, j\right) b\left(n_{r c}+1, k\right) \frac{1}{(2 l-1)} \\
& \left.-\sum_{j=1}^{n_{r c}+2} \sum_{k=1}^{n_{r c}+2} \sum_{l=1}^{k+j} b\left(n_{r c}+3, j\right) b\left(n_{r c}+3, k\right) \frac{1}{(2 l-1)}\right] \\
& +\frac{A^{2}\left(n_{r c}+3\right)^{2}\left(n_{r c}+1\right)^{2}}{3} \\
& \times\left[2 \sum_{j=1}^{n_{r c}+2} \sum_{k=1}^{n_{r c}} \sum_{l=1}^{k+j} b\left(n_{r c}+3, j\right) b\left(n_{r c}+1, k\right) \frac{2^{-l+1 / 2}}{(2 l-1)}\right. \\
& -\sum_{j=1}^{n_{r c}} \sum_{k=1}^{n_{r c}} \sum_{l=1}^{k+j} b\left(n_{r c}+1, j\right) b\left(n_{r c}+1, k\right) \frac{2^{-l+1 / 2}}{2 l-1} \\
& \left.-\sum_{j=1}^{n_{r c}+2} \sum_{k=1}^{n_{r c}+2} \sum_{l=1}^{k+j} b\left(n_{r c}+3, j\right) b\left(n_{r c}+3, k\right) \frac{2^{-l+1 / 2}}{2 l-1}\right] \\
& +\frac{2 A^{2}\left(n_{r c}+1\right)^{2}\left(n_{r c}+3\right)^{2}}{15}\left[\sum_{k=1}^{n_{r c}} \sum_{j=1}^{n_{r c}} b\left(n_{r c}+1, k\right) b\left(n_{r c}+1, j\right)\right. \\
& \left(2^{-k-j-3 / 2}+\frac{2 k+2 j+1}{2} \sum_{l=1}^{k+j+1} \frac{2^{-l+1 / 2}}{2 l-1}\right) \\
& +\sum_{k=1}^{n_{r c}+2} \sum_{j=1}^{n_{r c}+2} b\left(n_{r c}+3, j\right) b\left(n_{r c}+3, k\right)\left(2^{-k-j-3 / 2}\right. \\
& \left.+\frac{2 k+2 j+1}{2} \sum_{l=1}^{k+j+1} \frac{2^{-l+1 / 2}}{2 l-1}\right) \\
& -2 \sum_{k=1}^{n_{r c}} \sum_{j=1}^{n_{r c}+2} b\left(n_{r c}+1, k\right) b\left(n_{r c}+3, j\right)\left(2^{-l-j-3 / 2}\right. \\
& \left.+\frac{2 k+2 j+1}{2} \sum_{l=1}^{k+j+1} \frac{2^{-l+1 / 2}}{2 l-1}\right)
\end{aligned}
$$


where $b(n, i)=(-1)^{i}\left(4 n^{2}-2^{2}\right)\left(4 n^{2}-4^{2}\right) \ldots\left(4 n^{2}-4 i^{2}\right) /(2 i+1) !$. For large $\lambda$, we have analogous to (4.7)

$$
C_{\lambda(\lambda-2) / 4} \simeq-\frac{4}{\pi}[Z(\gamma+\ln (4 \lambda+10))-(Z+1)] .
$$

These values provided by the simple analytical expressions in (4.6) and (4.9) are marked by crosses on Fig. 7 to show that they provide an excellent approximation to the most negative eigenvalue.

\subsubsection{An Analytical Pair-Rydberg Formula A first approx-} imation to radial equations for the pair of electrons is provided by inserting the lowest eigenvalue of $\mathrm{C}$ for each $\lambda$ into (3.4). This provides potential wells,

$$
U(R)=\frac{\left(\lambda+\frac{3}{2}\right)\left(\lambda+\frac{5}{2}\right)}{2 R^{2}}+\frac{C}{R}
$$

with the effective charge $\mathrm{C}$ drawn from (4.6) and (4.9). As sketched in Fig. 12 , these potential wells converge to the double ionization limit. Each well describes a six-dimensional Coulomb problem and supports an infinity of discrete states. Singling out the lowest of these in each well provides a sequence of pair states described by the Rydberg expression

$$
E_{\lambda}=-\frac{C^{2}}{2\left(\lambda+\frac{5}{2}\right)^{2}}
$$

where the $5 / 2$ in the denominator is characteristic of the six-dimensions of the pair's hyperspherical space. For $\lambda=0,4,8 \ldots$, this Rydberg formula provides the approximation for what could be loosely described as the $N s^{2}$ configuration, and for $\lambda=2,6,10 \ldots$ for the $N p^{2}$ configuration.

In Table 4.4 we compare the results obtained from this simple analytical expression with those from other theoretical calculations for the lowest ridge states in $\mathrm{H}^{-}$and He. The results of Ho and Ho and Callaway [51] represent 


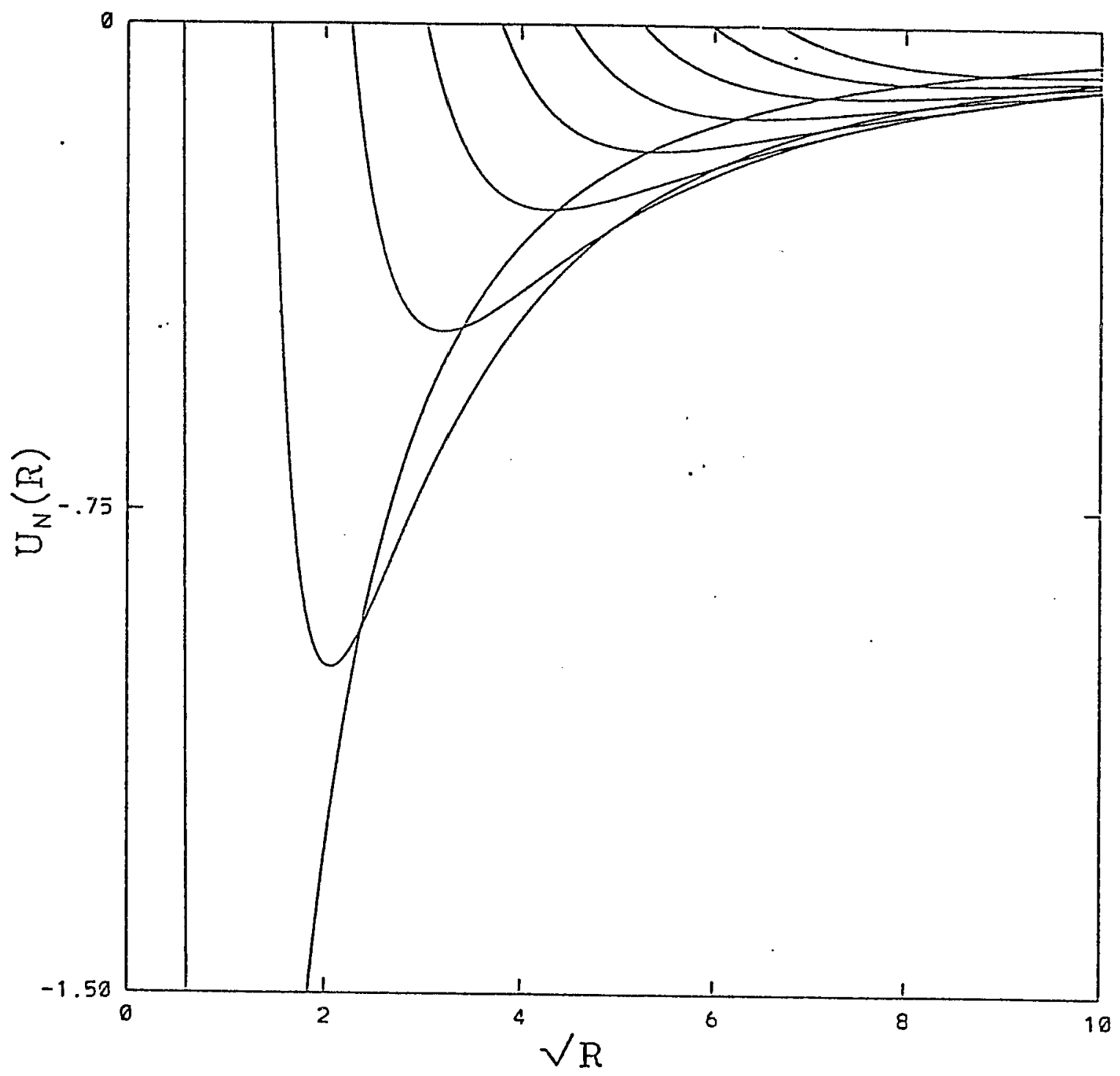

Figure 12. Potential Wells $\mathrm{U}(\mathrm{R})=\frac{(\lambda+3 / 2)(\lambda+5 / 2)}{2 R^{2}}+\frac{C_{\lambda \lambda / 4}}{R}$, with $\lambda=0,4,8,12,16,20$, $24,28,32$ and $\mathrm{Z}=2$. 
Table 4.4. Eigenvalues calculated by equation (4.12) for He.

$\begin{array}{lll}n(K, T)_{N}^{A} & \underline{\text { Present }} & \text { Ref. } 48 \\ 1(0,0)_{1}^{+} & -2.50000 & -2.89065 \\ 2(1,0)_{2}^{+} & -0.84135 & -0.77305 \\ 3(2,0)_{3}^{+} & -0.43006 & -0.35290 \\ 4(3,0)_{4}^{+} & -0.26700 & -0.2013 \\ 5(4,0)_{5}^{+} & -0.18447 & -0.12995 \\ 6(5,0)_{6}^{+} & -0.13633 & -0.09075\end{array}$

the best available from large numerical calculations with a basis of independent electron functions, whereas those of Rost and Briggs [48] represent the only calculation other than ours which also has a potential well converging to the double ionization limit. (They obtain this curve through scaling from the molecular $H_{2}^{+}$potential.) Our results are not as accurate as those obtained by these other authors for the low lying states. Our method is really adapted to describe the very highest states and we compare with the low ones only because they are the only data available (experimental data is even more sparse). Also, in the next section we will improve on our results in Table 4.4 by coupling multiple values of $\lambda$, but the specific distinction of the present results is the analytical Rydberg expression in (4.12). It is noteworthy that the dependence on the quantum number $\lambda$ is not just in the Bohr-Rydberg $\lambda^{-2}$ form but also through the dependence of the charge on $\lambda$ as given in (4.7) and (4.10). This slow logarithmic enhancement of the binding over the standard Rydberg value may be related to the overlapping of manifolds that has been observed in experiment [4-24] and in theoretical calculations [82] which group states below 
successive parental ionization thresholds (the parental threshold energies follow a standard Rydberg trend).

4.2.4 Coupled Potential Wells Retaining only the lowest eigenvalue at each $\lambda$ and considering each independently to give the potential in (4.12) is, of course, only a gross first approximation, and not expected to be accurate because of all the couplings that have been ignored. We proceed now to the next stage of off-diagonal coupling in $\lambda$. To take full account of this coupling we would have to consider several $\lambda$ manifolds and all the eigenvectors in each, and evaluate matrix elements of $C\left(\alpha, \theta_{12}\right)$ between them. But, as in the adiabatic hyperspherical method, where the lowest potential wells seem to play a dominant role $[30,74,81-83]$, we will also continue to retain only the lowest eigenvalue for each $\lambda$. But we will now calculate the off-diagonal matrix elements $C_{\lambda \lambda}$, between them. With the wave functions given in (4.5), these off-diagonal terms can also be evaluated analytically to give an expression analogous to (4.6),

$$
\begin{gathered}
C_{\lambda \lambda^{\prime}}=-\frac{8 Z}{\pi} \sum_{k=\frac{1}{2}\left|\lambda-\lambda^{\prime}\right|+1}^{\frac{1}{2}\left(\lambda+\lambda^{\prime}\right)+2} \frac{1}{2 k-1}+\frac{4 \sqrt{2}}{\pi} \sum_{k=\frac{1}{2}\left|\lambda-\lambda^{\prime}\right|+1}^{\frac{1}{2}\left(\lambda+\lambda^{\prime}\right)+2} \frac{(-)^{\left[\frac{k}{2}\right]}}{2 k-1} \\
\simeq-\frac{4 Z}{\pi} \ln \frac{\lambda+\lambda^{\prime}+5}{\left|\lambda-\lambda^{\prime}\right|+1} .
\end{gathered}
$$

Here (4.14) entails a further approximation for large $\lambda$ and $\lambda^{\prime}$ as before (a further small correction may be included if $\left|\lambda-\lambda^{\prime}\right|$ is not large). Similarly with the wavefunction given in (4.8), we can get the off diagonal terms for odd $\lambda / 2$;

$$
\begin{aligned}
\left\langle\lambda^{\prime}\left|C\left(\alpha, \theta_{12}\right)\right| \lambda\right\rangle= & -\frac{A A^{\prime}}{3} Z\left(n_{r c}+1\right)\left(n_{r c}+3\right)\left(n_{r c}^{\prime}+1\right)\left(n_{r c}^{\prime}+3\right) \\
& {\left[\sum_{j=1}^{n_{r c}+2} \sum_{k=1}^{n_{r c}^{\prime}} \sum_{l=1}^{k+j} b\left(n_{r c}+3, j\right) b\left(n_{r c}^{\prime}+1, k\right) \frac{1}{2 l-1}\right.}
\end{aligned}
$$




$$
\begin{aligned}
& +\sum_{j=1}^{n_{r c}^{\prime}+2} \sum_{k=1}^{n_{r c}} \sum_{l=1}^{k+j} b\left(n_{r c}^{\prime}+3, j\right) b\left(n_{r c}+1, k\right) \frac{1}{2 l-1} \\
& -\sum_{j=1}^{n_{r c}} \sum_{k=1}^{n_{r c}^{\prime}} \sum_{l=1}^{k+j} b\left(n_{r c}+1, j\right) b\left(n_{r c}^{\prime}+1, k\right) \frac{1}{2 l-1} \\
& \left.-\sum_{j=1}^{n_{r c}^{\prime}+2} \sum_{k=1}^{n_{r c}+2} \sum_{l=1}^{k+j} b\left(n_{r c}^{\prime}+3, j\right) b\left(n_{r c}+3, k\right) \frac{1}{2 l-1}\right] \\
& +\frac{A A^{\prime}}{3}\left(n_{r c}+1\right)\left(n_{r c}+3\right)\left(n_{r c}^{\prime}+1\right)\left(n_{r c}^{\prime}+3\right) \\
& +\sum_{j=1}^{n_{r c}+2} \sum_{k=1}^{n_{r c}^{\prime}} \sum_{l=1}^{k+j} b\left(n_{r c}+3, j\right) b\left(n_{r c}^{\prime}+1, k\right) \frac{2^{-l+1 / 2}}{2 l-1} \\
& +\sum_{j=1}^{n_{r c}^{\prime}+2} \sum_{k=1}^{n_{r c}} \sum_{l=1}^{k+j} b\left(n_{r c}^{\prime}+3, j\right) b\left(n_{r c}+1, k\right) \frac{2^{-l+1 / 2}}{2 l-1} \\
& +\sum_{j=1}^{n_{r c}} \sum_{k=1}^{n_{r c}^{\prime}} \sum_{l=1}^{k+j} b\left(n_{r c}+1, j\right) b\left(n_{r c}^{\prime}+1, k\right) \frac{2^{-l+1 / 2}}{2 l-1} \\
& +\frac{2 k+2 j}{n_{r c}+2} \sum_{j=1}^{n_{r c}^{\prime}+2} b+j \\
& +
\end{aligned}
$$




$$
\begin{aligned}
& -\sum_{k=1}^{n_{r c}^{\prime}} \sum_{j=1}^{n_{r c}+2} b\left(n_{r c}^{\prime}+1, k\right) b\left(n_{r c}+3, k\right)\left(2^{-k-j-3 / 2}\right. \\
& \left.\left.+\frac{2 k+2 j+1}{2} \sum_{l=1}^{k+j+1} \frac{2^{-l+1 / 2}}{2 l-1}\right)\right] .
\end{aligned}
$$

Proceeding in this manner, by retaining $\lambda$ values from 0 to some $\lambda_{\max }$ and diagonalizing the matrix given by (4.6) and (4.13) at each $\mathrm{R}$, gives potential wells $U_{N}(R)$ as shown in Fig. 13. These calculations which require diagonalization of $31 \times 31$ matrices are numerically straightforward because the matrix elements themselves are calculated analytically. In Fig. 13, a mesh for $\mathrm{R}$ with 1600 points was chosen and the calculation took only a few minutes on the IBM 3090 computer. To solve for the eigenvalues of doubly excited states, the eigenvectors obtained above at each $\mathrm{R}$ provide a basis $\psi_{N}(R ; \Omega)$ for expansion of the full wave function in (3.4)

$$
\Psi(R, \Omega)=\sum_{N} F_{N}(R) \psi_{N}(R ; \Omega)
$$

Inserting this into the Schrödinger equation in (3.4) leads as usual, and as in the close-coupling or adiabatic hyperspherical schemes, to coupled equations for $\vec{F}(R)$. The coupling between different $\mathrm{N}$ is provided by the matrix elements $<\psi_{N}\left|\frac{d}{d R}\right| \psi_{N^{\prime}}>$ and $<\psi_{N}\left|\frac{d^{2}}{d R^{2}}\right| \psi_{N^{\prime}}>$. In this chapter, we drop all these couplings off-diagonal in $\mathrm{N}$ in which case the eigenvalue problem reduces to that of calculating the states in each potential well in Fig. 13 independently

$$
\left[\frac{1}{2} \frac{d^{2}}{d R^{2}}-U_{N}(R)+E\right] R^{5 / 2} F_{N}(R)=0 .
$$

Eigenvalues obtained in this manner for $\mathrm{H}^{-}$and He are shown in Tables 4.5 and 4.6 for even $\lambda / 2$ and Tables 4.7 and 4.8 for odd $\lambda / 2$ and they represent improvements over the similar numbers in Table 4.4. The coupling of different $\lambda$ converges quickly, as shown in Tables 4.9 and 4.10 which contrast 


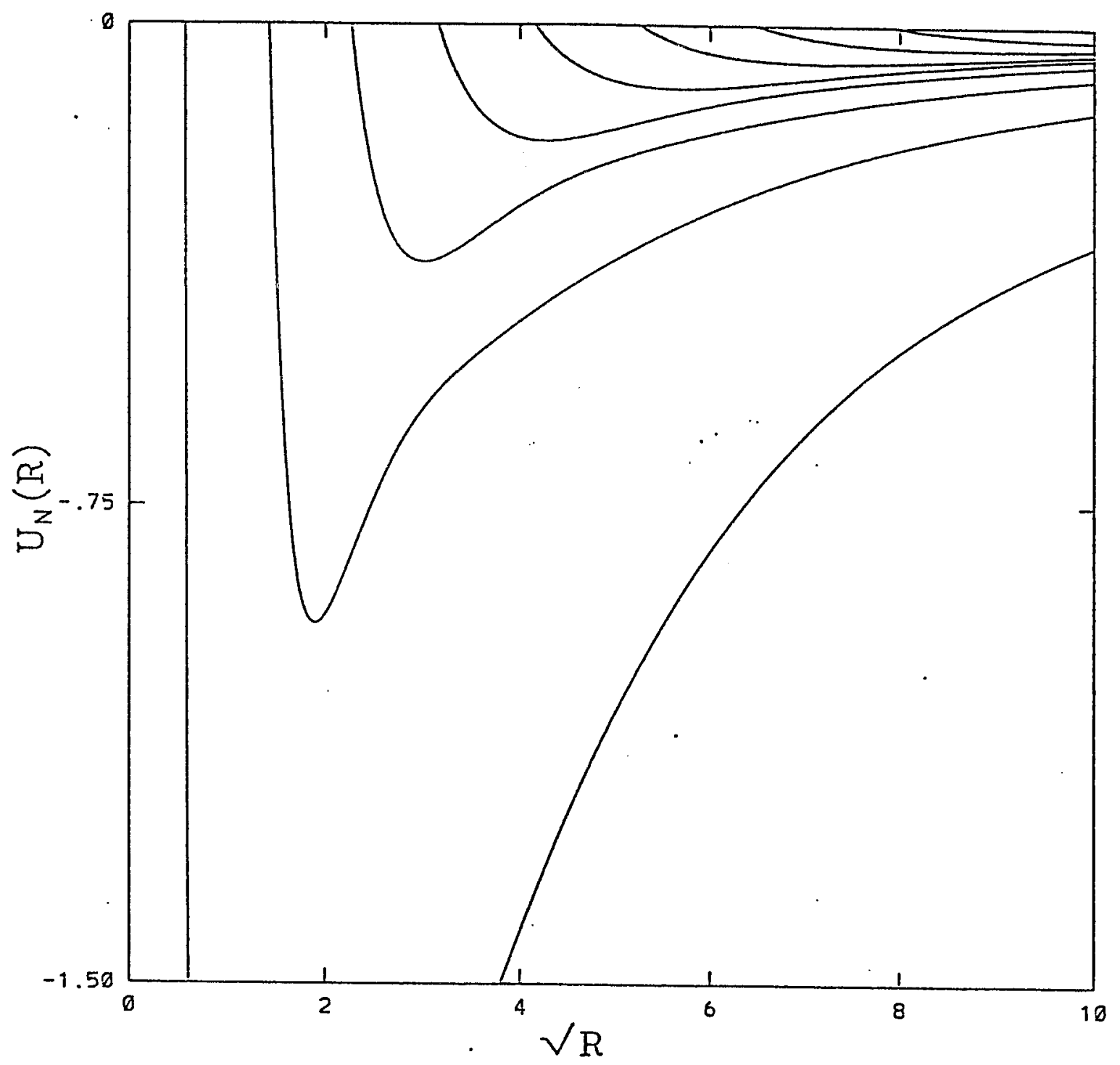

Figure 13. Potential curves by diagonalizing the matrix given by (4.6) and (4.13) with the same $\lambda$ values as in Fig. 12, but mutually coupled together. 
Table 4.5. Eigenvalues for the $H^{-}\left({ }^{1} S^{e}(1)\right)$ calculated by equation (4.17), upon coupling even values of $\lambda / 2$ up to $\lambda_{\max }=120$.

$\begin{array}{lllllll}n(K, T)_{N}^{A} & \text { present } & \text { Ref. } 81 & \underline{\text { Ref. } 69} & \underline{\text { Ref. } 51} & \underline{\text { Ref. } 49} & \text { exp. } \\ 1(0,0)_{1}^{+} & -0.52374 & -0.52590 & & & & \\ 2(1,0)_{2}^{+} & -0.13068 & -0.14879 & -0.14632 & -0.14878 & -0.14864 & -0.14864[19] \\ 3(2,0)_{3}^{+} & -0.05786 & -0.0696 & -0.06901 & -0.06900 & -0.06901 & -0.06875[19] \\ 4(3,0)_{4}^{+} & -0.03218 & -0.03993 & -0.03965 & -0.03960 & -0.03961 & \\ 5(4,0)_{5}^{+} & -0.02019 & -0.0260 & & -0.02570 & -0.02553 & \\ 6(5,0)_{6}^{+} & -0.01365 & -0.01821 & & -0.01800 & & \\ 7(6,0)_{7}^{+} & -0.00971 & -0.13525 & & -0.01333 & & \\ 8(7,0)_{8}^{+} & -0.00718 & -0.01042 & & -0.01203 & & \\ 9(8,0)_{9}^{+} & -0.00546 & -0.00827 & & -0.00814 & & \\ 10(9,0)_{10}^{+} & -0.00426 & & & & & \end{array}$

Table 4.6. Eigenvalues for the $\mathrm{He}\left({ }^{1} S^{e}(1)\right)$ calculated by equation (4.17), upon coupling even values of $\lambda / 2$ up to $\lambda_{\max }=120$.

\begin{tabular}{llllll} 
& \multicolumn{5}{c}{$\mathrm{He}^{1} S$} \\
$n(K, T)_{N}^{A}$ & present & $\underline{\text { Ref. } 48}$ & $\underline{\text { Ref. } 51}$ & $\underline{\text { Ref. 81 }}$ & exp. \\
$1(0,0)_{1}^{+}$ & -2.90582 & -2.89065 & & -2.8954 & -2.90372 \\
$2(1,0)_{2}^{+}$ & -0.75335 & -0.77305 & -0.77696 & -0.7721 & $-0.77799[8]$ \\
$3(2,0)_{3}^{+}$ & -0.33894 & -0.35290 & -0.35354 & -0.3529 & \\
$4(3,0)_{4}^{+}$ & -0.19037 & -0.20130 & -0.20105 & -0.20122 & \\
$5(4,0)_{5}^{+}$ & -0.12030 & -0.12995 & & -0.13028 & \\
$6(5,0)_{6}^{+}$ & -0.08170 & -0.09075 & & -0.09084 & \\
$7(6,0)_{7}^{+}$ & -0.05830 & -0.06695 & & -0.0675 & \\
$8(7,0)_{8}^{+}$ & -0.04320 & -0.05140 & & & \\
$9(8,0)_{9}^{+}$ & -0.03295 & -0.04073 & & & \\
$10(9,0)_{10}^{+}$ & -0.02570 & -0.03306 & & &
\end{tabular}


Table 4.7. Eigenvalues for the $H^{-}\left({ }^{1} S^{e}(2)\right)$ calculated by equation (4.17), upon coupling odd values of $\lambda / 2$ up to $\lambda_{\max }=120$.

\begin{tabular}{|c|c|c|c|c|c|}
\hline \multirow[b]{2}{*}{$n(K, T)_{N}^{+}$} & \multicolumn{5}{|c|}{$H^{-1} S(2)$} \\
\hline & present & Ref. 69 & Ref. 51 & Ref. 49 & Ref. 81 \\
\hline $2(-1,0)_{2}^{+}$ & -0.12343 & -0.12594 & & -0.12585 & \\
\hline $3(0,0)_{3}^{+}$ & -0.05425 & -0.05778 & & -0.05777 & -0.0558 \\
\hline $4(1,0)_{4}^{+}$ & -0.02961 & -0.0343 & -0.34725 & -0.03469 & \\
\hline $5(2,0)_{5}^{+}$ & -0.01881 & & -0.23315 & & -0.02317 \\
\hline $6(3,0)_{6}^{+}$ & -0.01204 & & -0.16665 & & -0.01661 \\
\hline $7(4,0)_{7}^{+}$ & -0.00894 & & -0.01252 & & -0.12505 \\
\hline $8(5,0)_{8}^{+}$ & -0.00652 & & -0.00972 & & -0.00975 \\
\hline $9(6,0)_{9}^{+}$ & -0.00490 & & -0.00775 & & -0.0078 \\
\hline $10(7,0)_{10}^{+}$ & -0.00376 & & & & \\
\hline
\end{tabular}

Table 4.8. Eigenvalues for the $\mathrm{He}\left({ }^{1} S^{e}(2)\right)$ calculated by equation (4.17), upon coupling odd values of $\lambda / 2$ up to $\lambda_{\max }=120$.

\begin{tabular}{llll} 
& \multicolumn{3}{c}{$\operatorname{He}^{1} S(2)$} \\
$n(K, T)_{N}^{+}$ & present & Ref. 81 & Ref. 51 \\
$2(-1,0)_{2}^{+}$ & -0.64470 & -0.60530 & -0.62192 \\
$3(0,0)_{3}^{+}$ & -0.30562 & -0.30719 & -0.31745 \\
$4(1,0)_{4}^{+}$ & -0.17572 & -0.18327 & -0.18785 \\
$5(2,0)_{5}^{+}$ & -0.11360 & -0.12103 & \\
$6(3,0)_{6}^{+}$ & -0.07920 & -0.08572 & \\
$7(4,0)_{7}^{+}$ & -0.05094 & & \\
$8(5,0)_{8}^{+}$ & -0.04301 & & \\
$9(6,0)_{9}^{+}$ & -0.03417 & & \\
$10(7,0)_{10}^{+}$ & -0.02594 & &
\end{tabular}


Table 4.9. Eigenvalues for He calculated by equation (4.17), with even values of $\lambda / 2$ and $\lambda_{\max }=320$ and $\lambda_{\max }=480$.

$\begin{array}{lll}n(K, T)_{N}^{A} & \lambda_{\max }=320 & \lambda_{\max }=480 \\ 1(0,0)_{1}^{+} & -2.90595 & -2.90595 \\ 2(1,0)_{2}^{+} & -0.75379 & -0.75381 \\ 3(2,0)_{3}^{+} & -0.34010 & -0.34016 \\ 4(3,0)_{4}^{+} & -0.19250 & -0.19269 \\ 5(4,0)_{5}^{+} & -0.12350 & -0.12374 \\ 6(5,0)_{6}^{+} & -0.08570 & -0.08604 \\ 7(6,0)_{7}^{+} & -0.06270 & -0.06318 \\ 8(7,0)_{8}^{+} & -0.04770 & -0.04827 \\ 9(8,0)_{9}^{+} & -0.03740 & -0.03800 \\ 10(9,0)_{10}^{+} & -0.03000 & -0.03063\end{array}$

results for $\lambda_{\max }=320$ and 480 . As expected, the lower states converge more quickly, and remain unaffected as the calculation embraces larger $\lambda$. A further slight shift takes place in the eigenvalues upon coupling all values of $\lambda / 2$ together, even and odd. These are displayed in Table 4.11, indexed by a pair principal quantum number $\nu$ and the pair quantum number $v(=(N-K-1) / 2)$ which describes angular correlations [35,74].

\subsection{Discussion}

In this chapter, we have shown that the diagonalization at fixed $\lambda$ can be carried out once and for all and even simple analytical expressions derived for the dominant eigenvalues. Extension to non-diagonal couplings in $\lambda$ is also almost as straightforward. We have presented evidence that the resulting potential wells $U_{N}(R)$ and basis function $\psi_{N}(R ; \Omega)$ already give a good accounting of the organization of doubly excited states into sequences converging to the double ionization limit, along with their energy positions. 
Table 4.10. Eigenvalues for He calculated by equation (4.17), with odd values $\lambda / 2$ and $\lambda_{\max }=320$ and $\lambda_{\max }=480$.

$\begin{array}{lll}n(K, T)_{N}^{+} & \lambda_{\max }=320 & \lambda_{\max }=480 \\ 2(-1,0)_{2}^{+} & -0.64471 & -0.64471 \\ 3(0,0)_{3}^{+} & -0.30562 & -0.30562 \\ 4(1,0)_{+}^{+} & -0.17575 & -0.17575 \\ 5(2,0)_{5}^{+} & -0.11370 & -0.11370 \\ 6(3,0)_{6}^{+} & -0.07945 & -0.07946 \\ 7(4,0)_{7}^{+} & -0.05859 & -0.05862 \\ 8(5,0)_{8}^{+} & -0.04492 & -0.04498 \\ 9(6,0)_{9}^{+} & -0.03550 & -0.03561\end{array}$

Table 4.11. Eigenvalues for the He calculated by coupling even and odd values of $\lambda / 2$ together with $\lambda_{\max }=120$.

$$
\begin{array}{lll}
\underline{\nu} & \underline{\mathrm{v}} & \text { present } \\
1 & 0 & -2.92688 \\
2 & 0 & -0.81327 \\
2 & 1 & -0.60447 \\
3 & 0 & -0.37117 \\
3 & 1 & -0.27919 \\
4 & 0 & -0.20968 \\
4 & 1 & -0.01587
\end{array}
$$


The next step of the program will be to consider the major couplings that have been left out, namely those arising from derivative terms $\frac{d}{d R}$ and $\frac{d^{2}}{d R^{2}}$ between the $\psi_{N}$ basis states. We will return to this in the next chapter, but note that the numerical procedure to handle coupled equations in a single variable $R$ is now standard. We will also extend the calculations to states of symmetry other than the ${ }^{1} S$ we have considered in this chapter. 


\section{CHAPTER 5}

\section{ELECTRON-PAIR ANALYSIS FOR DOUBLY EXCITED RIDGE STATES II: $\mathbf{L}=1$}

\subsection{Introduction}

In the previous chapter we restricted ourselves to the $\mathrm{L}=0$ case when the equations (3.8)-(3.11) simplify dramatically as in (4.1)-(4.3). The equations are algebraically more complicated when $l \neq 0$. When $\mathrm{L}=1$, two cases arise $:$ if $l_{1} \neq l_{2}$ with $\left|l_{1}-l_{2}\right|=1$, then $\lambda\left(=l_{1}+l_{2}+2 n_{r c}\right)$ and the states have odd parity, whereas if $l_{1}=l_{2}$, then $\lambda\left(=l_{1}+l_{2}+2 n_{r c}\right)$ and the states have even parity. In the next section, we will discuss even and odd parity states separately.

\subsection{Calculational Procedure}

5.2.1 $P^{o}$ States $\quad$ In ${ }^{1} P^{o}$ states, the two individual angular momenta $l_{1}$ and $l_{2}$ are not equal, and, $Y_{l_{1} l_{2} 10}$ does not simplify as in the $L=0$ case. The wavefunction in this case is

$$
\Phi_{n_{r c} l_{1} l_{2}}(\Omega)=\frac{1}{\sqrt{2}}\left[\phi_{n_{r c} l_{1} l_{2} 10}(\Omega)+(-)^{n_{r c}+S} \phi_{n_{r c} l_{2} l_{1} 10}(\Omega)\right],
$$

with $S=0$ for singlet states and $S=1$ for triplet states. Since the coupled spherical harmonics does not have a simple analytical expression, the 
calculation of the matrix elements becomes more complicated than before. The matrix elements in each $\lambda$ subspace are

$$
<\Phi_{n_{r c} l_{1} l_{2}}(\Omega)\left|C\left(\alpha, \theta_{12}\right)\right| \Phi_{n_{r c}^{\prime} l_{1}^{\prime} l_{2}^{\prime}}(\Omega)>
$$

It is useful to divide the charge operator into

$$
C\left(\alpha, \theta_{12}\right)=U(\alpha)+W\left(\alpha, \theta_{12}\right)
$$

with

$$
\begin{aligned}
U(\alpha) & =-\frac{Z}{\cos \alpha}-\frac{Z}{\sin \alpha} \\
W\left(\alpha, \theta_{12}\right) & =\left[1-\sin 2 \alpha \cos \theta_{12}\right]^{-1 / 2} .
\end{aligned}
$$

Then

$$
\begin{aligned}
\left.<\Phi_{n_{r c} l_{1} l_{2}}\left|C\left(\alpha, \theta_{12}\right)\right| \Phi_{n_{r c}^{\prime} l_{1}^{\prime} l_{2}^{\prime}}\right\rangle= & \iint d \Omega C\left(\alpha, \theta_{12}\right) \Phi_{n_{r c} l_{1} l_{2}} \Phi_{n_{r c}^{\prime} l_{1}^{\prime} l_{2}^{\prime}} \\
= & \int_{0}^{\frac{\pi}{2}} d \alpha \frac{\sin ^{2} 2 \alpha}{4} U(\alpha) \Phi_{n_{r c} l_{1} l_{2}} \Phi_{n_{r c}^{\prime} l_{1}^{\prime} l_{2}^{\prime}} \delta_{l_{1}^{\prime} l_{1}} \delta_{l_{2}^{\prime} l_{2}} \\
& +\iint d \Omega W\left(\alpha, \theta_{12}\right) \Phi_{n_{r c} l_{1} l_{2}} \Phi_{n_{r c}^{\prime} l_{1}^{\prime} l_{2}^{\prime}}
\end{aligned}
$$

We have dropped the indices $S, \pi, \mathrm{L}$ and $\mathrm{M}$ because these values are already fixed. Only the second term in (5.5) involves the coupling between the coupled spherical harmonics. We will briefly outline how to calculate this coupling. Expanding $W\left(\alpha, \theta_{12}\right)$ into multipoles in $\theta_{12}$ in the form

$$
\begin{aligned}
{\left[1-\sin 2 \alpha \cos \theta_{12}\right]^{-1 / 2} } & =\sum_{k=0}^{\infty} \frac{\tan ^{k} \alpha}{\cos \alpha} P_{k}\left(\cos \theta_{12}\right), \quad 0 \leq \alpha \leq \frac{\pi}{4} \\
& =\sum_{k=0}^{\infty} \frac{\cot ^{k} \alpha}{\sin \alpha} P_{k}\left(\cos \theta_{12}\right), \quad \frac{\pi}{4} \leq \alpha \leq \frac{\pi}{2}
\end{aligned}
$$

we define $A\left[k, l_{1}, l_{2}, l_{1}^{\prime}, l_{2}^{\prime}\right]$ as follows

$$
A\left[k, l_{1}, l_{2}, l_{1}^{\prime}, l_{2}^{\prime}\right]=<Y_{l_{1} l_{2} 10}\left|P_{k}\left(\cos \theta_{12}\right)\right| Y_{l_{1}^{\prime} l_{2}^{\prime} 10}>
$$




$$
\begin{aligned}
& =(-)^{l_{1}^{\prime}+l_{2}+L}<l_{1}\left\|C^{k}|| l_{1}^{\prime}><l_{2}\right\| C^{k} \| l_{2}^{\prime}> \\
& \times\left\{\begin{array}{lll}
l_{1} & l_{2} & L \\
l_{2}^{\prime} & l_{1}^{\prime} & k
\end{array}\right\},
\end{aligned}
$$

where

$$
<l_{i} \| C^{k}|| l_{j}>=(-) \sqrt[l_{i}]{\left(2 l_{i}+1\right)\left(2 l_{j}+1\right)}\left(\begin{array}{ccc}
l_{i} & k & l_{j} \\
0 & 0 & 0
\end{array}\right)
$$

and $\left(\begin{array}{ccc}l_{i} & k & l_{j} \\ 0 & 0 & 0\end{array}\right)$ is a $3-\mathrm{j}$ symbol. If $l_{i}+k+l_{j}=2 g, g$ an integer and it has also satisfied the triangular relation $\triangle\left(l_{i} k l_{j}\right)$, the 3 - $\mathrm{j}$ symbol can be evaluated $[93-94]$ as in (3.16)

$$
\left(\begin{array}{ccc}
l_{i} & k & l_{j} \\
0 & 0 & 0
\end{array}\right)=(-) \sqrt[g]{\frac{\left(2 g-2 l_{i}\right) !(2 g-2 k) !\left(2 g-2 l_{j}\right) !}{(2 g+1) !}} \frac{g !}{\left(g-l_{i}\right) !(g-k) !\left(g-l_{j}\right) !} .
$$

If $l_{i}+k+l_{j}=2 g+1$, the $3-\mathrm{j}$ symbol is zero. $\left\{\begin{array}{lll}l_{1} & l_{2} & L \\ l_{2}^{\prime} & l_{1}^{\prime} & k\end{array}\right\}$ is a 6 - $\mathrm{j}$ symbol as defined in (3.19)-(3.22). Using such standard expressions for these symbols [93-94], we get

$$
\begin{aligned}
A\left[k, l_{1}, l_{2}, l_{1}^{\prime}, l_{2}^{\prime}\right]= & (-)^{l_{1}^{\prime}+1+g_{1}+g_{2}+l_{1}} \sqrt{\left(2 l_{1}+1\right)\left(2 l_{2}+1\right)\left(2 l_{1}^{\prime}+1\right)\left(2 l_{2}^{\prime}+1\right)} \\
& \times \sqrt{\frac{\left(2 g_{1}-2 l_{1}\right) !\left(2 g_{1}-2 k\right) !\left(2 g_{1}-2 l_{1}^{\prime}\right) !}{\left(2 g_{1}+1\right) !}} \\
& \times \frac{g_{1} !}{\left(g_{1}-l_{1}\right) !\left(g_{1}-k\right) !\left(g_{1}-l_{1}^{\prime}\right) !} \\
& \times \sqrt{\frac{\left(2 g_{2}-2 l_{2}\right) !\left(2 g_{2}-2 k\right) !\left(2 g_{2}-2 l_{2}^{\prime}\right) !}{\left(2 g_{2}+1\right) !}} \\
& \times \frac{g_{2} !}{\left(g_{2}-l_{2}\right) !\left(g_{2}-k\right) !\left(g_{2}-l_{2}^{\prime}\right) !} B(X),
\end{aligned}
$$


Table 5.1. Eigenvalues of effective charge operator $C_{\lambda Q}$ for ${ }^{1} P^{o}$ states of $\mathrm{He}$, where $\lambda \leq 11$.

\begin{tabular}{lllllll}
$\lambda \backslash \mathrm{Q}$ & 0 & 1 & 2 & 3 & 4 & 5 \\
\hline 1 & -5.35011 & & & & & \\
3 & -4.62453 & -6.92450 & & & & \\
5 & -4.33924 & -5.80663 & -7.55965 & & & \\
7 & -4.16930 & -5.34584 & -6.26637 & -8.31188 & & \\
9 & -4.05000 & -5.07998 & -5.71090 & -6.87170 & -8.70776 & \\
11 & -3.95790 & -4.90139 & -5.38690 & -6.22412 & -7.19248 & -9.21080
\end{tabular}

where $g_{1}=\left(l_{1}+l_{1}^{\prime}+k\right) / 2, g_{2}=\left(l_{2}+l_{2}^{\prime}+k\right) / 2$, and

$$
B(X)=(-)^{X}\left[\frac{X(X+1)(X-2 k-1)(X-2 k)}{\left(2 l_{1}-1\right) 2 l_{1}\left(2 l_{1}+1\right)\left(2 l_{1}^{\prime}-1\right) 2 l_{1}^{\prime}\left(2 l_{1}^{\prime}+1\right)}\right]^{\frac{1}{2}},
$$

if $X=k+l_{1}+l_{1}^{\prime}, l_{2}=l_{1}-1$ and $l_{2}^{\prime}=l_{1}^{\prime}-1$ and the same expression with subscripts 1 replaced by 2 if $X=k+l_{1}+l_{1}^{\prime}+2, l_{2}=l_{1}+1, l_{2}^{\prime}=l_{1}^{\prime}+1$, whereas

$$
B(X)=(-)^{X}\left[\frac{\left(X-2 l_{1}^{\prime}\right)\left(X-2 l_{1}^{\prime}-1\right)\left(X-2 l_{1}+1\right)\left(X-2 l_{1}+2\right)}{\left(2 l_{1}^{\prime}+1\right)\left(2 l_{1}^{\prime}+2\right)\left(2 l_{1}^{\prime}+3\right)\left(2 l_{1}-1\right) 2 l_{1}\left(2 l_{1}+1\right)}\right]^{\frac{1}{2}},
$$

if $X=k+l_{1}+l_{1}^{\prime}, l_{2}=l_{1}-1$ and $l_{2}^{\prime}=l_{1}^{\prime}+1$, with primed and unprimed entries interchanged when $X=k+l_{1}+l_{1}^{\prime}, \quad l_{2}=l_{1}+1$ and $l_{2}^{\prime}=l_{1}^{\prime}-1$.

Inserting the coupling coefficient $\mathrm{A}$ into (5.5), and evaluating the $\alpha$ integrand numerically, we can diagonalize the matrix in (5.2). The eigenvalues $C_{\lambda Q}$ of this matrix are tabulated in Table 5.1 and the eigenvalues plotted $v s$ the quantum number $Q$ in Fig. 14 for $\lambda \leq 27$. The eigenvalues are labeled by $\mathrm{Q}=0,1, \ldots ;$ the maximum value of $\mathrm{Q}$ is $\frac{1}{2}(\lambda-1)$.

5.2.2 Eigenvalues for ${ }^{1} P^{\circ}$ States As discussed in the previous chapter, the extreme eigenvalues are well approximated by the diagonal matrix elements with the lowest and highest $n_{r c}$ values, also now for $L=1$ states. In 


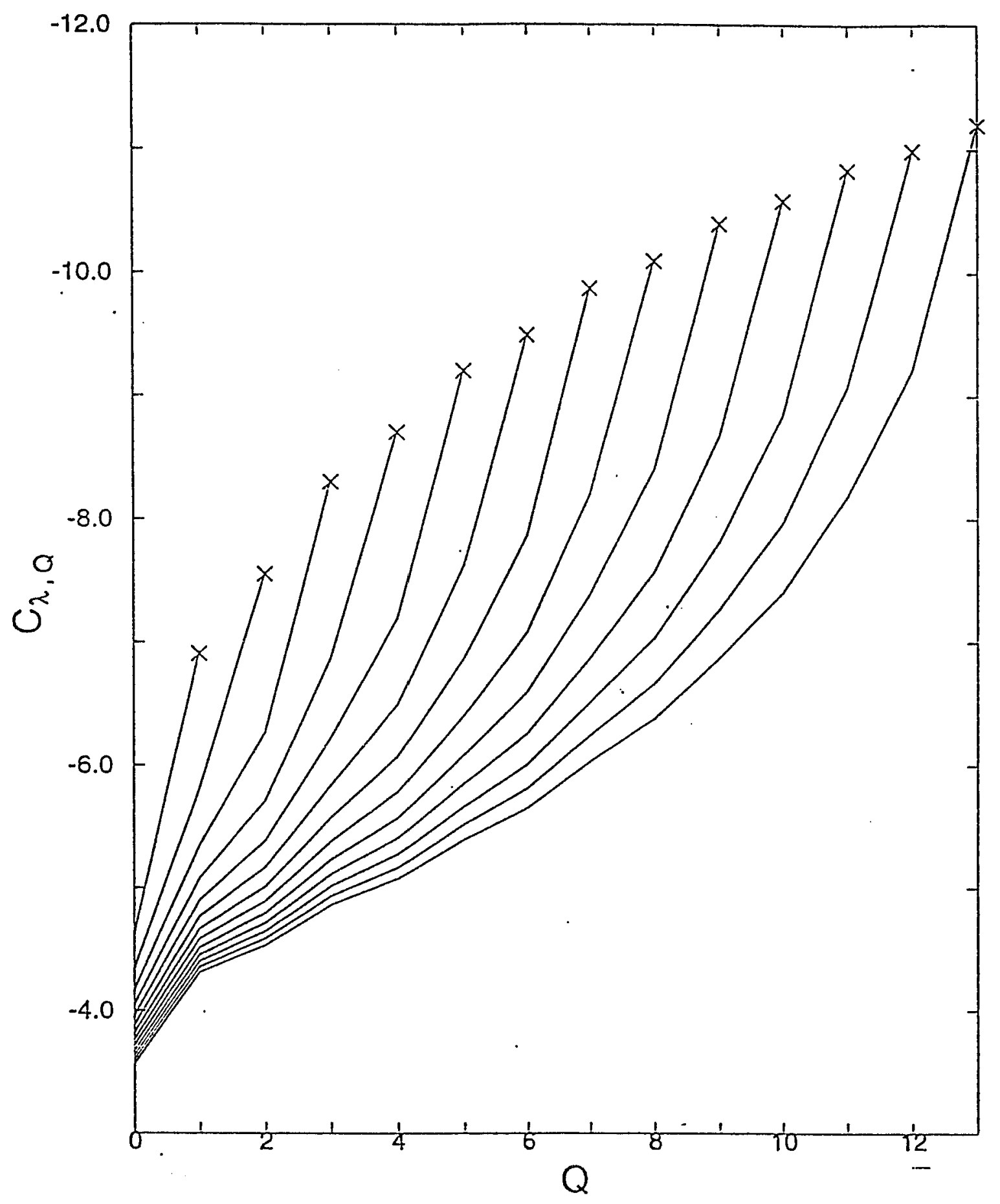

Figure 14. Eigenvalues $C_{\lambda Q}$ for ${ }^{1} P^{o}$ states of He at fixed $\lambda$. Results of numerical diagonalization of (5.5) are shown as continuous curves. Crosses give maximum eigenvalues as given by the approximate, analytical expression in (5.15). 
either of these cases, $\Phi$ in (3.9) takes a simple form and the matrix element can be evaluated analytically. We are interested only in the lowest eigenvalue for each $\lambda$ to get the most attractive potential. This has $l_{1}=1, l_{2}=0$, and the highest value of $n_{r c}=\frac{1}{2}(\lambda-1)$. When $n_{r c}$ is even, the corresponding states are called $n_{+}$, and when $n_{r c}$ is odd, the corresponding states are called $n_{-}$. The wavefunction, therefore, reduces to

$$
\begin{aligned}
\Phi_{\lambda n_{r c}}(\Omega)= & N_{\lambda n_{r c}}\left\{\frac { 1 } { \operatorname { c o s } \alpha \operatorname { s i n } 2 \alpha } \left[\left(n_{r c}+2\right) \sin 2\left(n_{r c}+1\right) \alpha+\right.\right. \\
& \left.\left(n_{r c}+1\right) \sin 2\left(n_{r c}+2\right) \alpha\right] Y_{1010}\left(\hat{r}_{1}, \hat{r}_{2}\right) \\
& +(-)^{n_{r c}+S} \frac{1}{\sin \alpha \sin 2 \alpha}\left[\left(n_{r c}+2\right) \sin 2\left(n_{r c}+1\right) \alpha\right. \\
& \left.\left.-\left(n_{r c}+1\right) \sin 2\left(n_{r c}+2\right) \alpha\right] Y_{0110}\left(\hat{r}_{1}, \hat{r}_{2}\right)\right\}
\end{aligned}
$$

where $N_{\lambda n_{r c}}=2 /\left[\left(n_{r c}+1\right)\left(n_{r c}+2\right) \pi\right]^{1 / 2}$ is the normalization constant. The evaluation of matrix elements of $\mathrm{C}$

$$
C_{\lambda Q=(\lambda-1) / 2}=<\Phi_{\lambda n_{r c}}(\Omega)\left|C\left(\alpha, \theta_{12}\right)\right| \Phi_{\lambda n_{r c}}(\Omega)>
$$

analytically requires some algebra. The diagonal term is given by

$$
\begin{aligned}
C_{\lambda Q=(\lambda-1) / 2}= & -Z \frac{4}{\pi}\left(n_{r c}+1\right)\left(n_{r c}+2\right)\left[-4-4 \sum_{j=1}^{n_{r c}} \sum_{k=1}^{j+1} b\left(n_{r c}+1, j\right) \frac{1}{2 k-1}\right. \\
& -4 \sum_{j=1}^{n_{r c}+1} \sum_{k=1}^{j+1} b\left(n_{r c}+2, j\right) \frac{1}{2 k-1} \\
& -\sum_{j=1}^{n_{r c}} \sum_{k=1}^{n_{r c}} \sum_{i=1}^{j+k+1} b\left(n_{r c}+1, k\right) b\left(n_{r c}+1, k\right) \frac{1}{2 i-1} \\
& -\sum_{j=1}^{n_{r c}+1} \sum_{k=1}^{n_{r c}+1} \sum_{i=1}^{j+k+1} b\left(n_{r c}+2, j\right) b\left(n_{r c}+2, k\right) \frac{1}{2 i-1} \\
& -2 \sum_{j=1}^{n_{r c}} \sum_{k=1}^{n_{r c}+1} \sum_{i=1}^{j+k+1} b\left(n_{r c}+1, j\right) b\left(n_{r c}+2, k\right) \frac{1}{2 i-1} \\
& +\sum_{j=1}^{n_{r c}} \sum_{k=1}^{n_{r c}} b\left(n_{r c}+1, j\right) b\left(n_{r c}+1, k\right) \frac{1}{2 k+2 j+1}
\end{aligned}
$$




$$
\begin{aligned}
& +\sum_{j=1}^{n_{r c}+1} \sum_{k=1}^{n_{r c}+1} b\left(n_{r c}+2, j\right) b\left(n_{r c}+2, k\right) \frac{1}{2 j+2 k+1} \\
& \left.-2 \sum_{j=1}^{n_{r c}} \sum_{k=1}^{n_{r c+1}} b\left(n_{r c}+1, j\right) b\left(n_{r c}+2, k\right) \frac{1}{2 j+2 k+1}\right] \\
& +\frac{4}{\pi}\left(n_{r c}+1\right)\left(n_{r c}+2\right)[-2 \sqrt{2} \\
& -4 \sum_{j=1}^{n_{r c}} \sum_{k=1}^{j+1} b\left(n_{r c}+1, j\right) \frac{2^{-k+1 / 2}}{2 k-1} \\
& +4 \sum_{j=1}^{n_{r c}+1} \sum_{k=1}^{j+1} b\left(n_{r c}+2, j\right) \frac{2^{-k+1 / 2}}{2 k-1} \\
& +\sum_{j=1}^{n_{r c}} \sum_{k=1}^{n_{r c}} \sum_{k=1}^{j+k+1} b\left(n_{r c}+1, j\right) b\left(n_{r c}+1, k\right) \frac{2^{-i+1 / 2}}{2 i-1} \\
& +\sum_{j=1}^{n_{r c}+1} \sum_{j=1}^{n_{r=1}} \sum_{j=1}^{n_{r c}} \sum_{j=1}^{n_{r c}+1} \sum_{j=1}^{k+1} b\left(n_{r c}+1\right. \\
& +
\end{aligned}
$$


For large $\lambda$, this expression for the effective charge can be simplified further as

$$
C_{\lambda Q=(\lambda-1) / 2} \simeq-\frac{4}{\pi}\left[Z\left(\left(\gamma-\frac{1}{2}+\ln (4 \lambda+10)\right)-1\right] .\right.
$$

With this effective charge, we have potential wells for each of the $\lambda$ values as displayed in Fig. 15.

5.2.3 $P^{e}$ States In $P^{e}$ states, the two individual angular momenta $l_{1}$ and $l_{2}$ are equal. The wavefunction in this case is (for $\mathrm{M}=0$ )

$$
\Phi_{n_{r c} l_{1} l_{2} L M}^{S \pi}(\Omega)=\Phi_{n_{r c} l l 10}^{S \pi}(\Omega)=\frac{1}{2}\left[1+(-1)^{S+1+n_{r c}}\right] f_{n_{r c} l l}(\alpha) Y_{l l 10}\left(\hat{r}_{1}, \hat{r}_{2}\right) .
$$

For singlet states, $n_{r c}$ is odd, and for triplet states, it is even. From (3.10) and (3.11), we have

$$
\begin{gathered}
f_{n_{r c} l l}(\alpha)=N_{n_{r c} l l} \cos ^{l} \alpha \sin ^{l} \alpha_{2} F_{1}\left(-n_{r c}, n_{r c}+2 l+2, l+\frac{3}{2} ; \sin ^{2} \alpha\right) . \\
Y_{l l 10}\left(\hat{r}_{1}, \hat{r}_{2}\right)=\sum_{m_{1} m_{2}}\left(l l 10 \mid l m_{1} l m_{2}\right) Y_{l m_{1}}\left(\hat{r}_{1}\right) Y_{l m_{2}}\left(\hat{r}_{2}\right)
\end{gathered}
$$

with $l \neq 0$. Upon diagonalizing the effective charge operator $C\left(\alpha, \theta_{12}\right)$ in a $\lambda$ subspace, we get eigenvalues for ${ }^{1} P^{e}$ states as shown in Table 5.2. The plot of the eigenvalues $v s$ the quantum number $Q$ in Fig. 16. The maximum $Q$ value is $\frac{1}{4}(\lambda-4)$. The most attractive eigenvalue we are interested in has the highest $n_{r c}$. In ${ }^{1} P^{e}$ states, this means $n_{r c}=(\lambda-2) / 2, l_{1}=l_{2}=1$. The wavefunction has a simple form

$$
\begin{aligned}
\Phi_{\lambda n_{r c}}\left(\alpha, \theta_{12}\right)= & N_{\lambda n_{r c}} \frac{1}{\sin ^{2} 2 \alpha}\left[\left(n_{r c}+3\right) \sin 2\left(n_{r c}+1\right) \alpha\right. \\
& \left.-\left(n_{r c}+1\right) \sin 2\left(n_{r c}+3\right) \alpha\right] Y_{l 110}\left(\hat{r}_{1}, \hat{r}_{2}\right) .
\end{aligned}
$$

where

$$
N_{\lambda n_{r c}}=2 /\left[\left(n_{r c}+1\right)\left(n_{r c}+3\right) \pi\right]^{1 / 2} .
$$




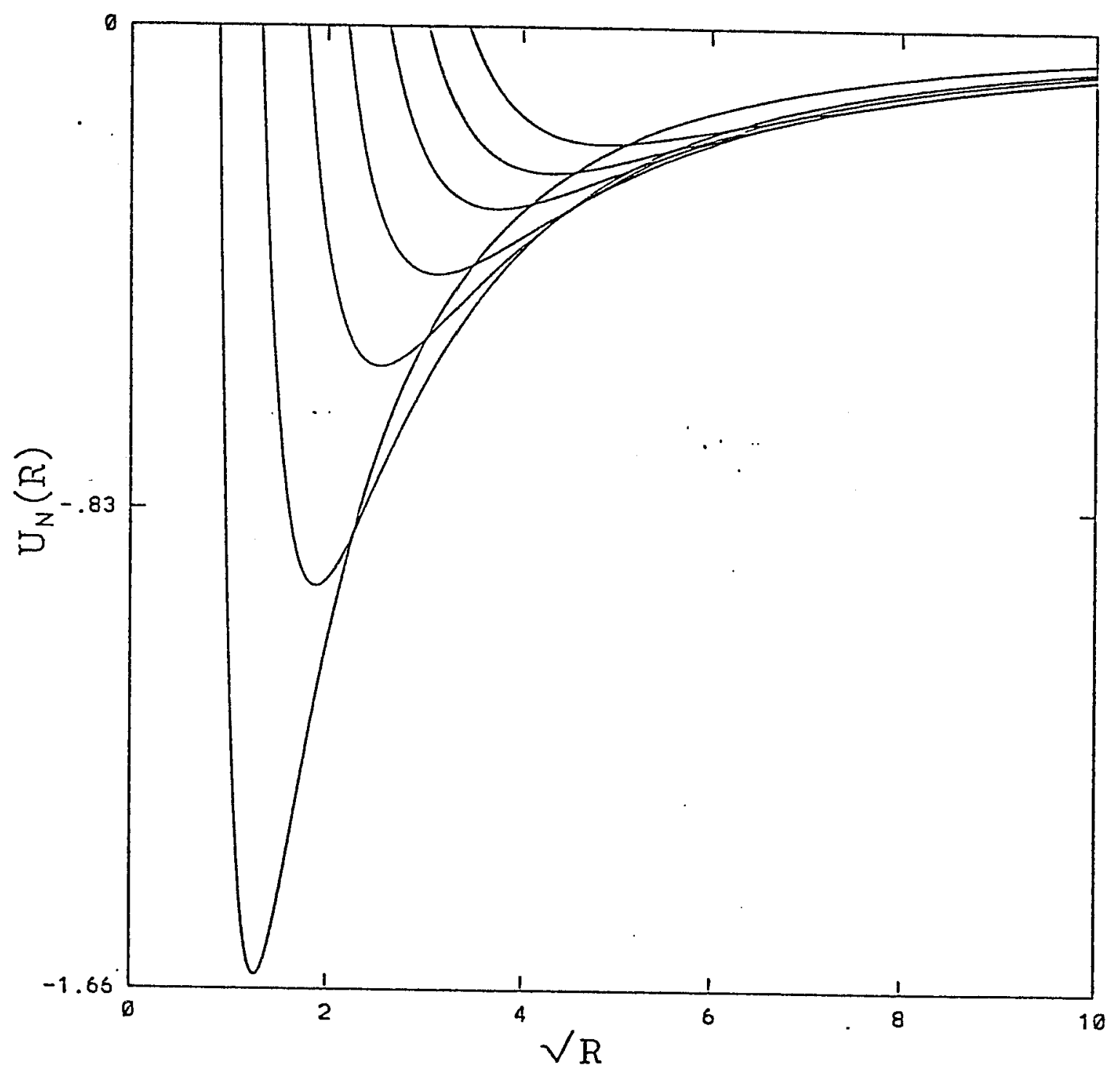

Figure 15. Potential wells $U(R)=\frac{(\lambda+3 / 2)(\lambda+5 / 2)}{2 R^{2}}+\frac{C_{\lambda(\lambda-1) / 2}}{R}$, with $\lambda=1,3,5,7$, $9,11,13$ and $\mathrm{Z}=2$, for ${ }^{1} P^{\circ}$. 


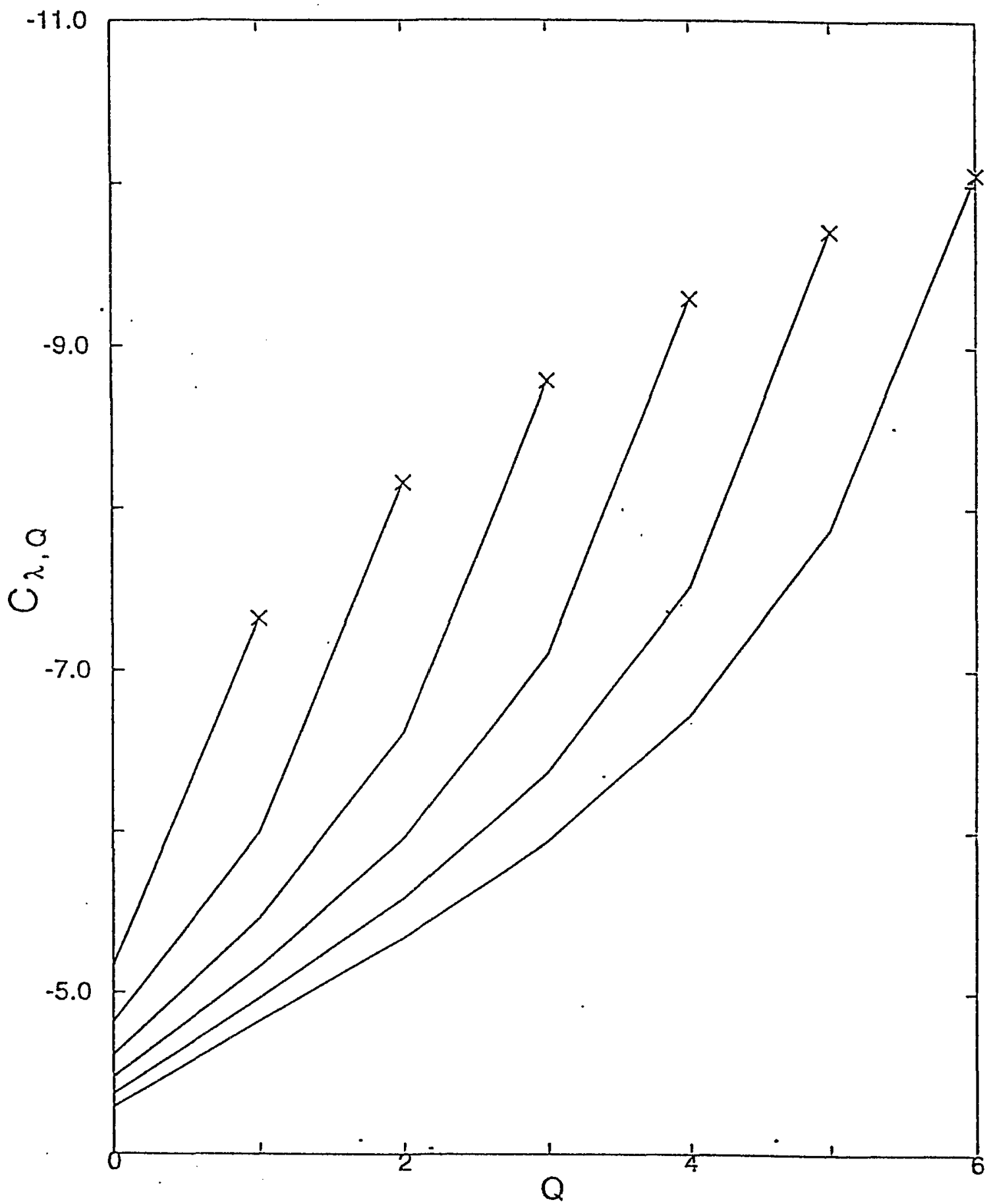

Figure 16. Eigenvalues for ${ }^{1} P^{e}$ states in rie. Results of numerical diagonalization of (5.5) are shown as continuous curves. Crosses give maximum eigenvalues as given by the approximate, analytical expression in (5.23). 
Table 5.2. Eigenvalues of effective charge operator $C_{\lambda Q}$ for ${ }^{1} P^{e}$ states of He, where $\lambda \leq 24$.

\begin{tabular}{lllllll}
$\lambda \backslash \mathrm{Q}$ & 0 & 1 & 2 & 3 & 4 & 5 \\
\hline 4 & -6.08498 & & & & & \\
8 & -5.17068 & -7.32564 & & & & \\
12 & -4.81808 & -5.99767 & -8.15896 & & & \\
16 & -4.61589 & -5.46740 & -6.61824 & -8.15896 & & \\
20 & -4.47798 & -5.16878 & -5.96346 & -7.10498 & -9.29145 & \\
24 & -4.37441 & -4.97164 & -5.59152 & -6.37359 & -7.51786 & -9.71256
\end{tabular}

$$
C_{\lambda Q=\frac{\lambda-4}{4}}=<\Phi_{\lambda n_{r c}}(\Omega) \mid C\left(\alpha, \theta_{12} \mid \Phi_{\lambda n_{r c}}(\Omega)>.\right.
$$

The analytical expression is

$$
\begin{aligned}
C_{\lambda Q=(\lambda-4) / 4}= & \frac{-2 Z}{\pi}\left(n_{r c}+1\right)\left(n_{r c}+3\right) \\
& {\left[2 \sum_{j=1}^{n_{r c}+2} \sum_{k=1}^{n_{r c}} \sum_{i=1}^{k+j} b\left(n_{r c}+3, j\right) b\left(n_{r c}+1, k\right) \frac{1}{2 i-1}\right.} \\
& -\sum_{k=1}^{n_{r c}} \sum_{j=1}^{n_{r c}} \sum_{i=1}^{k+j} b\left(n_{r c}+1, j\right) b\left(n_{r c}+1, k\right) \frac{1}{2 i-1} \\
& \left.-\sum_{k=1}^{n_{r c}+2} \sum_{j=1}^{n_{r c}+2} \sum_{i=1}^{k+j} b\left(n_{r c}+3, j\right) b\left(n_{r c}+3, k\right) \frac{1}{2 i-1}\right] \\
& \frac{2}{\pi}\left(n_{r c}+1\right)\left(n_{r c}+3\right)[ \\
& 2 \sum_{j=1}^{n_{r c}+2} \sum_{k=1}^{n_{r c}} \sum_{i=1}^{j+k} b\left(n_{r c}+3, j\right) b\left(n_{r c}+1, k\right) \frac{2^{-i+1 / 2}}{2 i-1} \\
& -\sum_{j=1}^{n_{r c}} \sum_{k=1}^{n_{r c}} \sum_{i=1}^{k+j} b\left(n_{r c}+1, j\right) b\left(n_{r c}+1, k\right) \frac{2^{-i+1 / 2}}{2 i-1} \\
& \left.-\sum_{j=1}^{n_{r c}+2} \sum_{k=1}^{n_{r c}+2} \sum_{i=1}^{k+j} b\left(n_{r c}+3, j\right) b\left(n_{r c}+3, k\right) \frac{2^{-i+1 / 2}}{2 i-1}\right] \\
& -\frac{2}{5 \pi}\left(n_{r c}+1\right)\left(n_{r c}+3\right)\left[\sum _ { k = 1 } ^ { n _ { r c } } \sum _ { j = 1 } ^ { n _ { r c } } b ( n _ { r c } + 1 , k ) b ( n _ { r c } + 1 , j ) \left(2^{-k-j-3 / 2}\right.\right.
\end{aligned}
$$




$$
\begin{aligned}
& \left.+\frac{2 k+2 j+1}{2} \sum_{i=1}^{k+j+1} \frac{2^{-i+1 / 2}}{2 i-1}\right) \\
& +\sum_{k=1}^{n_{r c}+2} \sum_{j=1}^{n_{r c}+2} b\left(n_{r c}+3, k\right) b\left(n_{r c}+3, j\right)\left(2^{-k-j-3 / 2}+\right. \\
& \left.\frac{2 k+2 j+1}{2} \sum_{i=1}^{k+j+1} \frac{2^{-i+1 / 2}}{2 i-1}\right) \\
& -2 \sum_{k=1}^{n_{r c}} \sum_{j=1}^{n_{r c}+2} b\left(n_{r c}+1, k\right) b\left(n_{r c}+3, j\right)\left(2^{-k-j-3 / 2}+\right. \\
& \left.\left.\frac{2 k+2 j+1}{2} \sum_{i=1}^{k+j+1} \frac{2^{-i+1 / 2}}{2 i-1}\right)\right]
\end{aligned}
$$

This effective charge can be simplified further for large $\lambda$ as

$$
C_{\lambda Q=\frac{\lambda-4}{4}} \simeq=-\frac{4}{\pi}[Z(\gamma-2+\ln (4 \lambda+10))+(Z-1)+0.15] .
$$

Using these effective charges, we get the potential wells for each $\lambda$ as shown in Fig. 17.

5.2.4 Coupled Potential Wells As we did in the previous chapter, in each $\lambda$ subspace we only retain the lowest eigenvalue as a first approximation. In order to get more accurate results we need to consider the coupling between different $\lambda$. As observed before, in each $\lambda$ subspace only the lowest potential wells seem to play a dominant role. Therefore, we will only keep the lowest eigenvalue for each $\lambda$. However, we now also calculate the off-diagonal matrix elements between different $\lambda$

$$
C_{\lambda \lambda^{\prime}}=<\Phi_{\lambda n_{r c}}|C(\alpha, \Omega)| \Phi_{\lambda^{\prime} n_{r c}^{\prime}}>
$$

For any $\lambda_{\max }$, we can diagonalize the matrix given by (5.15) and (5.25) by retaining $\lambda$ values from 1 to $\lambda_{\max }$ for ${ }^{1} P^{o}$ state and (5.23) and (5.25) by retaining $\lambda$ values from 4 to $\lambda_{\max }$ for ${ }^{1} P^{e}$ states at each $\mathrm{R}$ value. With the corresponding 


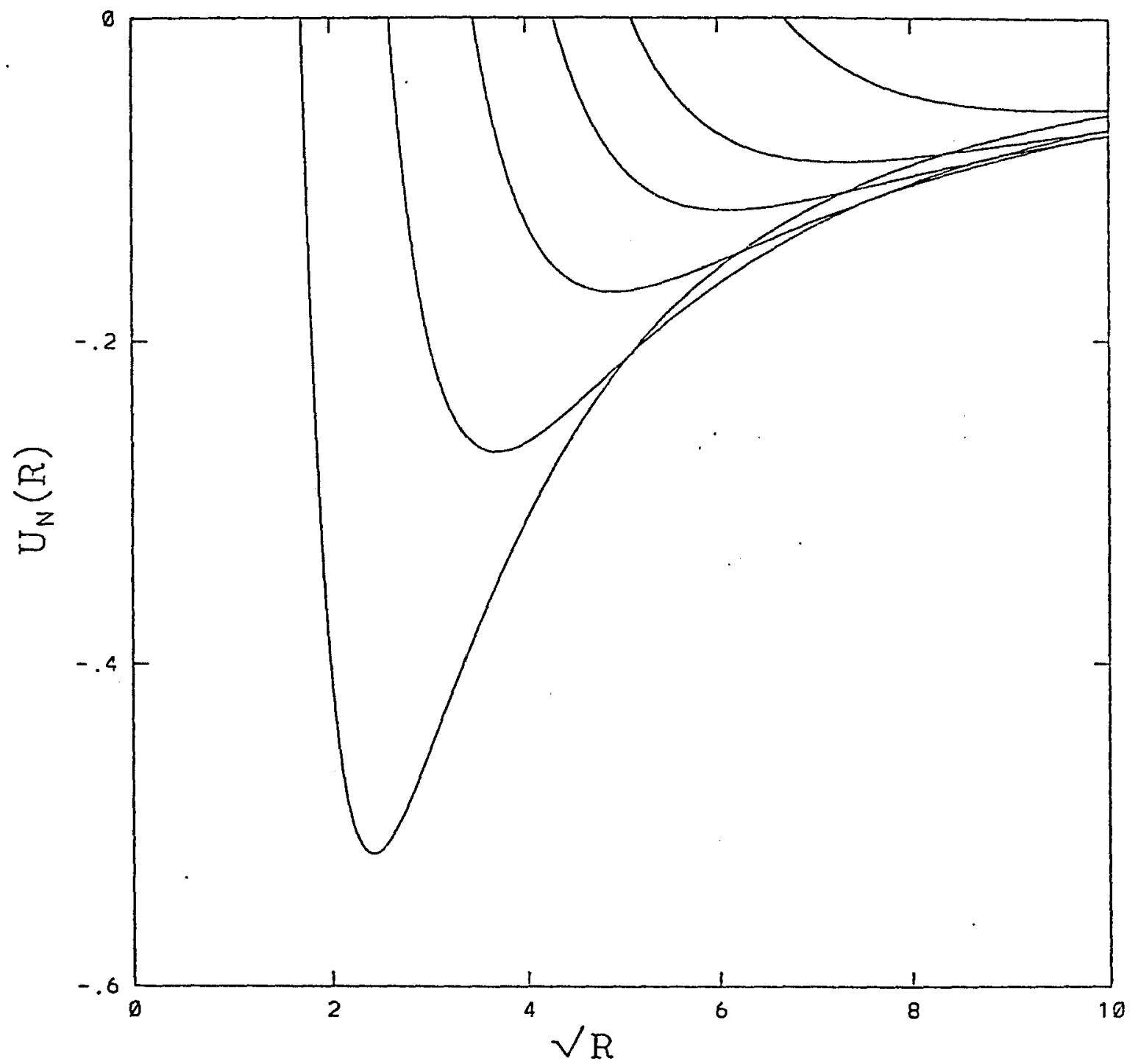

Figure 17. Potential wells $U(R)=\frac{(\lambda+3 / 2)(\lambda+5 / 2)}{2 R^{2}}+\frac{C_{\lambda(\lambda-2) / 2}}{R}$, with $\lambda=4,8,12$, $16,20,24,28$ and $\mathrm{Z}=2$, for ${ }^{1} P^{e}$. 
eigenvalues at each $\mathrm{R}$, we construct the potential wells $U_{N}(R)$ as shown in Fig. 18 for ${ }^{1} P^{o}$ and Fig. 19 for ${ }^{1} P^{e}$ states. If we choose $\lambda_{\max }=121$ we need to diagonalize numerically $61 \times 61$ matrix elements in ${ }^{1} P^{o}$ case and $30 \times 30$ in ${ }^{1} P^{e}$ case.

To solve for the eigenvalues of doubly excited states, the eigenvectors obtained at each $\mathrm{R}$ provide a basis $\psi_{N}(R ; \Omega)$ for expansion of the full wave function in (3.4)

$$
\Psi(R, \Omega)=\sum_{N} F_{N}(R) \psi_{N}(R ; \Omega)
$$

Inserting this into the Schrödinger equation (3.4) leads to coupled differential equations for $\vec{F}(R)$, or equivalently for $\vec{G}(R)=R^{5 / 2} \vec{F}(R)$, we have

$$
\sum_{N}\left[\frac{1}{2}\left(-\frac{d^{2}}{d R^{2}}+\frac{\Lambda^{2}+\frac{15}{4}}{R^{2}}\right)+\frac{C\left(\alpha, \theta_{12}\right)}{R}\right] G_{N}(R) \psi_{N}(R ; \Omega)=E \sum_{N} G_{N}(R) \psi_{N}(R ; \Omega) .
$$

Mutiplying by $\psi_{N^{\prime}}(R ; \Omega)$ and integrating over $\Omega$, we get

$$
\begin{aligned}
& \sum_{N N^{\prime}}-\frac{1}{2}\left[\frac{d^{2}}{d R^{2}} G_{N}(R) \delta_{N N^{\prime}}+2 \frac{d G_{N}}{d R}<\psi_{N^{\prime}}\left|\frac{d}{d R}\right| \psi_{N}>+<\psi_{N}\left|\frac{d^{2}}{d R^{2}}\right| \psi_{N^{\prime}}>G_{N}(R)\right] \\
& +U_{N}(R) G_{N}(R)=E G_{N}(R) .
\end{aligned}
$$

The equation can be written as

$$
\left[\left(\frac{d^{2}}{d R^{2}}+2 E\right) I-2 \mathbf{U}(\mathbf{R})+\mathbf{W}(\mathbf{R})\right] \vec{G}(R)=0
$$

where

$$
\mathbf{U}(\mathbf{R})=<\psi_{N}\left|\frac{\Lambda^{2}}{2 R^{2}}+\frac{C}{R}\right| \psi_{N}>
$$

and

$$
\mathbf{W}(\mathbf{R})=2<\psi_{N}\left|\frac{d}{d R}\right| \psi_{N^{\prime}}>\frac{d}{d R}+<\psi_{N}\left|\frac{d^{2}}{d R^{2}}\right| \psi_{N^{\prime}}>
$$




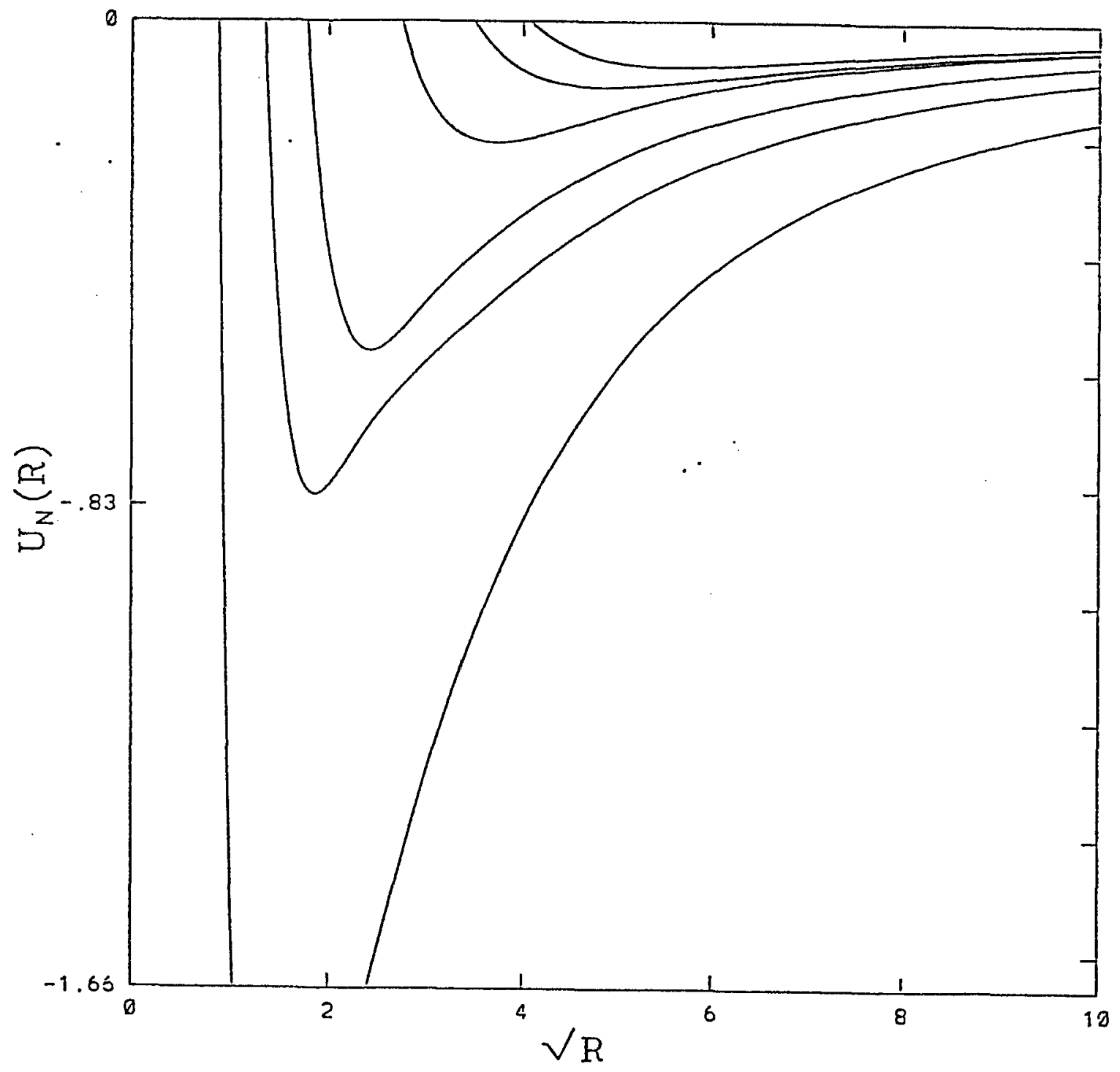

Figure 18. Potential curves for ${ }^{1} P^{\circ}$ by diagonalizing the matrix given by (5.15) and (5.25) with $\lambda=1,3,5,7,9,11,13$ and $\mathrm{Z}=2$. 


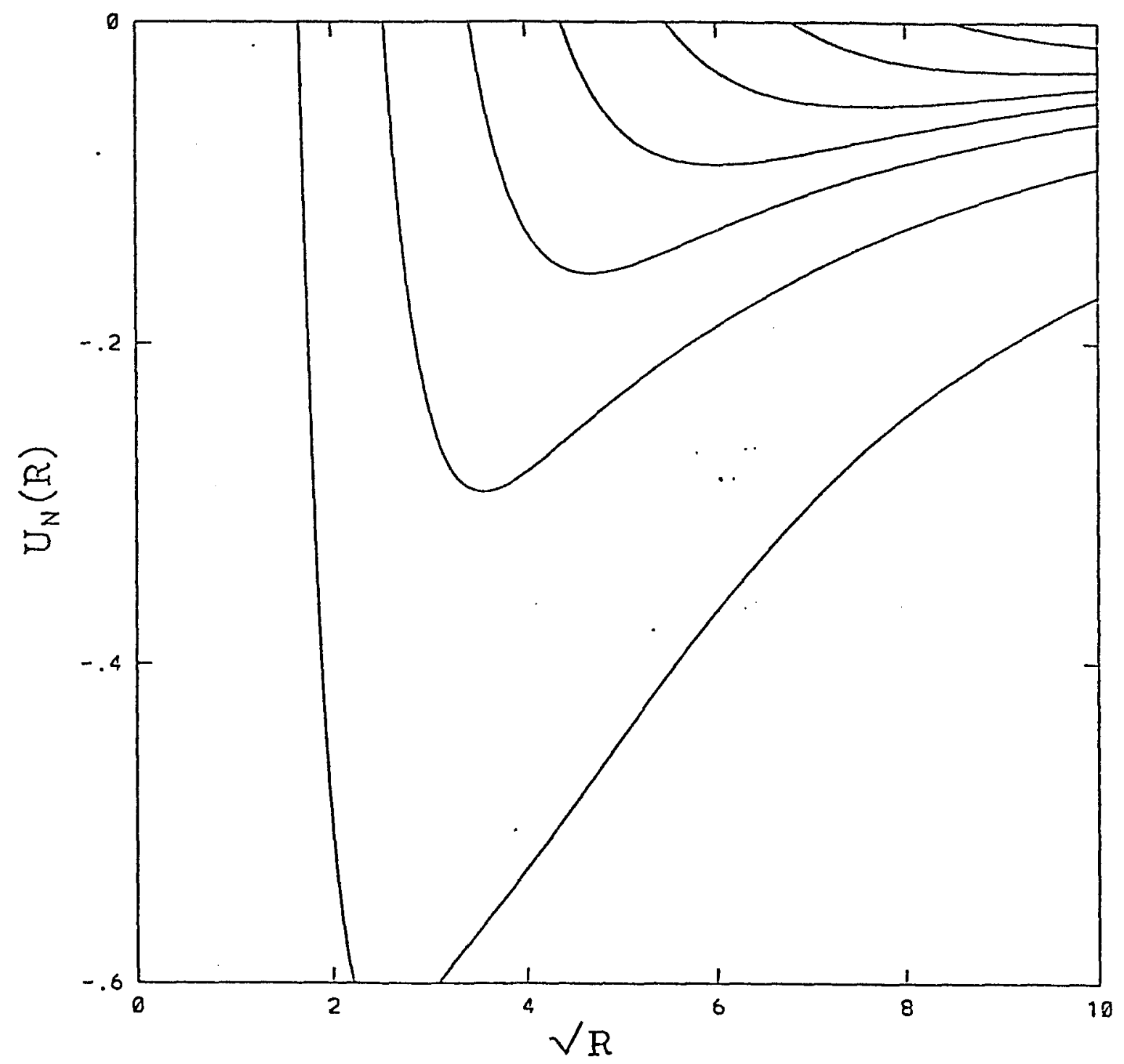

Figure 19. Potential curves for ${ }^{1} P^{e}$ by diagonalizing the matrix given by (5.23) and (5.25) with $\lambda=4,8,12,16,20,24,28$ and $Z=2$. 
Table 5.3. Eigenvalues calculated by equation (5.32) for $H^{-}$of ${ }^{1} \mathrm{P}^{\circ}$, where $\lambda_{\max }=121$.

\begin{tabular}{lllllll} 
& \multicolumn{5}{c}{$H^{-1} P^{o}$} \\
$n(K, T)_{N}^{A}$ & present & Other[Ref.] & $\underline{\text { Ref. 69 }}$ & Ref. 97 & Ref. 49 & exp. \\
${ }_{2}(0,0)_{1}^{0}$ & -0.48768 & & & & & \\
$2(0,1)_{2}^{+}$ & -0.12608 & $-0.12601[82]$ & -0.12605 & & -0.12604 & $-0.12813[17]$ \\
$3(1,0)_{2}^{-}$ & -0.11928 & $-0.12424[82]$ & -0.12440 & & -0.124328 & \\
${ }_{3}(1,1)_{3}^{+}$ & -0.05702 & $-0.06165[67]$ & -0.06272 & -0.06272 & -0.06271 & $-0.06258[20]$ \\
$4(2,0)_{3}^{-}$ & -0.05163 & $-0.05834[67]$ & -0.05857 & -0.05857 & -0.05857 & \\
$4(2,1)_{4}^{+}$ & -0.03282 & $-0.03678[82]$ & -0.03715 & -0.03718 & -0.03713 & $-0.03714[20]$ \\
$5(3,0)_{4}^{-}$ & -0.02798 & $-0.03423[63]$ & & -0.03429 & -0.03429 & \\
$5(3,1)_{5}^{+}$ & -0.02067 & $-0.02452[82]$ & & -0.02452 & & $-0.02452[20]$ \\
$6(4,0)_{5}^{-}$ & -0.01652 & $-0.02258[81]$ & & -0.02263 & & \\
${ }_{6}(4,1)_{6}^{+}$ & -0.01174 & $-0.01752[82]$ & & -0.01736 & & $-0.01733[20]$
\end{tabular}

The first approximation ignores all the couplings between different channels to get a set of uncoupled differential equations.

$$
\left[\frac{d^{2}}{d R^{2}}+2 E-2 U_{N}(R)\right] G(R)=0
$$

Each of the potential wells $U_{N}(R)$ converges to the double ionization limit as before in the case of ${ }^{1} S$ states. For each potential well, we can solve equation (5.32) numerically. We use the $5^{\text {th }}$ order Runge Kutta method to solve this equation. The eigenvalues are given in Table 5.3 for $\mathrm{H}^{-}$and $5.4 \mathrm{He}$ for ${ }^{1} \mathrm{P}^{\circ}$ and Table 5.5 for ${ }^{1} P^{e}$ states. The ${ }^{1} P^{o}$ states can be loosely described as the NsNp and (N-1)sNp configuration. Similarly ${ }^{1} P^{e}$ can be described as the $(\mathrm{N}-1) \mathrm{pNp}$ configuration. The more appropriate ${ }_{n}(K, T)_{N}^{A}$ lables are shown in the tables.

Now we discuss the effects of $\frac{d}{d R}$ and $\frac{d^{2}}{d R^{2}}$ couplings. The matrix $\mathbf{W}(\mathbf{R})$ which appear in (5.29) comes from the $\mathrm{R}$ dependence of $\psi_{N}$. Because the two 
Table 5.4. Eigenvalues calculated by equation (5.32) for $\mathrm{He}$ of ${ }^{1} P^{\circ}$, where $\lambda_{\max }=121$.

\begin{tabular}{lllll}
\multicolumn{5}{c}{ He $^{1} P^{o}$} \\
${ }_{n}(K, T)_{N}^{A}$ & present & Other[Ref.] & Ref. 97 & Exp. \\
${ }_{2}(0,0)_{1}^{O}$ & -2.13987 & $-2.12160[81]$ & & \\
${ }_{2}(0,1)_{2}^{+}$ & -0.67964 & $-0.6928[70]$ & -0.69313 & $-0.69298[3]$ \\
${ }_{3}(1,0)_{2}^{-}$ & -0.58762 & $-0.59707[70]$ & -0.59707 & \\
${ }_{3}(1,1)_{3}^{+}$ & -0.31172 & $-0.33760[81]$ & -0.33563 & $-0.33392[9]$ \\
${ }_{4}(2,0)_{3}^{-}$ & -0.27734 & $-0.27070[81]$ & -0.28595 & \\
${ }_{4}(2,1)_{4}^{+}$ & -0.17775 & $-0.19556[82]$ & -0.19454 & $-0.1944[7]$ \\
$5(3,0)_{4}^{-}$ & -0.16140 & $-0.16830[81]$ & -0.17882 & \\
$5(3,1)_{5}^{+}$ & -0.11440 & $-0.12799[82]$ & -0.12643 & $-0.1261[7]$ \\
$6(4,0)_{5}^{-}$ & -0.10477 & $-0.11155[81]$ & -0.11917 & \\
$6(4,1)_{6}^{+}$ & -0.07930 & $-0.08924[82]$ & -0.08860 & $-0.0881[7]$
\end{tabular}

Table 5.5. Eigenvalues calculated by equation (5.32) for He and $H^{-}$of ${ }^{1} P^{e}$, where $\lambda_{\text {max }}=120$.

$\mathrm{He}^{1} P^{e} \quad H^{-1} P^{e}$

${ }_{n}(K, T)_{N}^{A} \quad$ present $\quad$ Ref. 97 $\quad$ Other[Ref.] present Other[Ref.] Ref. 97

${ }_{3}(0,1)_{2}^{-} \quad-0.58398$

${ }_{4}(1,1)_{3}^{-} \quad-0.27790 \quad-0.27899$

${ }_{5}(2,1)^{-} \quad-0.16421 \quad-0.16552$

${ }_{6}(3,1)^{\frac{-}{5}} \quad-0.10776 \quad-0.10993$

$7(4,1)_{6}^{-} \quad-0.07567$

$8(5,1) \overline{7} \quad-0.05822$

$9(6,1)_{8}^{-} \quad-0.04061$
$-0.05625[67] \quad-0.12369$

$-0.05497-0.0560[49]$

$\begin{array}{lll}-0.03142 & -0.03305[49] & -0.03132\end{array}$

$-0.01937-0.02182$ 
matrices $\mathbf{U}$ and $\mathbf{W}$ do not commute, they cannot be diagonalized simultaneously. But in our case the $\mathrm{R}$ dependence comes wholly from the coefficients in $\psi_{N}(R ; \Omega)=\sum_{\lambda} a_{N \lambda}(R) \Phi_{\lambda n_{r c}}(\Omega)$. This property makes the problem much simpler; because $\left\langle\psi_{N} \mid \psi_{N^{\prime}}\right\rangle=\delta_{N N^{\prime}}$, we have $\left\langle\psi_{N}\right| \frac{d \psi_{N}}{d R}>=0$. Inclusion of the $\mathbf{W}(\mathbf{R})$ term in the Schrödinger equation gives a positive contribution to the energy. When we include this term in Schrödinger equation we will, therefore, get an upper bound on the energy. In our case $\mathbf{W}(\mathbf{R})$ can be expressed as

$$
\mathbf{W}(\mathbf{R})=\sum_{\lambda} a_{N \lambda}(R) \frac{d^{2}}{d R^{2}} a_{N^{\prime} \lambda}(R)+2 \sum_{\lambda} a_{N \lambda}(R) \frac{d}{d R} a_{N^{\prime} \lambda}(R) \frac{d}{d R}
$$

According to the Born-Oppenheimer approximation, if we neglect the offdiagonal terms in $W$ (actually these terms are very small, $10^{-10}$ ), the Schrödinger equation can be expressed as

$$
\left\{\frac{d^{2}}{d R^{2}}+W(R)+2(E-U(R))\right\} \vec{G}(R)=0 .
$$

Modifying the Runge Kutta algorithm, and solving equation (5.34) numerically, we get the eigenvalues shown in Table 5.6 for $\mathrm{He}$ and $H^{-}$in ${ }^{1} P^{\circ}$ states and 5.7 in ${ }^{1} P^{e}$.

\subsection{Results and Discussion}

Tables 5.1 and 5.2 give the diagonalization results for the effective charge operator in a $\lambda$ manifold. Table 5.3 gives the eigenvalues for $H^{-}$and $5.4 \mathrm{He}$ for ${ }^{1} P^{o}$ and Table 5.5 for ${ }^{1} P^{e}$, as calculated from (5.32) with individual potential wells. Tables 5.6 and 5.7 give the improved results from (5.34) including coupling between wells; these are upper bounds on the energies of the ${ }^{1} P^{o}$ and ${ }^{1} P^{e}$ states. Similarly, by coupling $\lambda=1,3,5 \ldots .121, l_{1}=1, l_{2}=0$ and $S=1$, we get the eigenvalues for ${ }^{3} P^{\circ}$ states, and upon coupling $\lambda=2,6,10 \ldots .118$, $\mathrm{S}=1, l_{1}=l_{2}=1$, and $n_{r c}=(\lambda-2) / 2$, we get the eigenvalues for ${ }^{3} P^{e}$ states. 
Table 5.6. Eigenvalues calculated by equation (5.34) for He and $H^{-}$of ${ }^{1} P^{o}$ state, where $\lambda_{\max }=121$ and $\frac{d}{d R}$ and $\frac{d^{2}}{d R^{2}}$ terms are included.

$\begin{array}{lll}{ }_{n}(K, T)_{N}^{A} & \mathrm{He} & H^{-} \\ { }_{2}(0,0)_{1}^{0} & -2.11552 & -0.48620 \\ { }_{2}(0,1)_{2}^{+} & -0.64131 & -0.12446 \\ { }_{3}(1,0)_{2}^{-} & -0.55077 & -0.11799 \\ 3(1,1)_{3}^{+} & -0.28463 & -0.05592 \\ { }_{4}(2,0)_{3}^{-} & -0.25345 & -0.05068 \\ 4(2,1)_{4}^{+} & -0.16262 & -0.03180 \\ 5(3,0)_{4}^{-} & -0.14761 & -0.02710 \\ 5(3,1)_{5}^{+} & -0.09843 & -0.01987 \\ 6(4,0)_{5}^{-} & -0.09753 & -0.01553 \\ 6(4,1)_{6}^{+} & -0.07499 & -0.01082\end{array}$

Table 5.7. Eigenvalues calculated by equation (5.34) for He and $H^{-}$of ${ }^{1} P^{e}$ state, where $\lambda_{\max }=120$ and $\frac{d}{d R}$ and $\frac{d^{2}}{d R^{2}}$ terms are included.

$$
\begin{array}{lll}
{ }_{n}(K, T)_{N}^{A} & \mathrm{He} & H^{-} \\
{ }_{3}(0,1)_{2}^{-} & -0.57902 & -0.12331 \\
{ }_{4}(1,1)_{3}^{-} & -0.27259 & -0.05489 \\
{ }_{5}(2,1)_{4}^{-} & -0.15997 & -0.03067 \\
6(3,1)_{5}^{-} & -0.10486 & -0.01873 \\
7(4,1)^{-} & -0.07392 & -0.01222 \\
8(5,1)^{-} & -0.05665 & \\
9(6,1)_{8}^{-} & -0.03989 &
\end{array}
$$


Table 5.8. Eigenvalues calculated by equation (5.32) for He and $H^{-}$of ${ }^{3} P^{o}$ states, where $\lambda_{\max }=121$.

\begin{tabular}{|c|c|c|c|c|c|c|}
\hline & \multicolumn{3}{|c|}{$\mathrm{He}^{3} P^{o}$} & \multicolumn{3}{|c|}{$H^{-3} P^{o}$} \\
\hline${ }_{n}(K, T)_{N}^{A}$ & present & Other[Ref.] & Ref. 97 & present & Other[Ref.] & Ref. 97 \\
\hline${ }_{2}(0,0)_{1}^{0}$ & -2.15607 & & & -0.48787 & & \\
\hline${ }_{2}(1,0)_{2}^{+}$ & -0.77875 & $-0.78196[83]$ & & -0.14271 & & \\
\hline${ }_{3}(0,1)_{2}^{-}$ & -0.57371 & $-0.58281[83]$ & & -0.11912 & $-0.12425[67]$ & \\
\hline${ }_{3}(2,0)_{3}^{+}$ & -0.36050 & $-0.35[69]$ & -0.35038 & -0.06712 & $-0.06828[63]$ & \\
\hline${ }_{4}(1,1)_{3}^{-}$ & -0.26546 & & -0.27948 & -0.05147 & $-0.0553[49]$ & \\
\hline${ }_{4}(3,0)_{4}^{+}$ & -0.20473 & & -0.20008 & -0.03839 & $-0.03959[49]$ & -0.03936 \\
\hline $5(2,1)_{4}^{-}$ & -0.15275 & & -0.16514 & -0.02784 & & \\
\hline${ }_{5}(4,0)_{5}^{+}$ & & & & -0.02438 & -0.025 & -0.02568 \\
\hline
\end{tabular}

Table 5.8 gives the eigenvalues for $\mathrm{He}$ and $\mathrm{H}^{-}$in ${ }^{3} \mathrm{P}^{o}$ states and Table 5.9 in ${ }^{3} P^{e}$.

Our $P^{o}$ results compare favorably with Ref. $49,63,67,69$ and 81 83. We also compare our $P^{e}$ results with Ho's [97] latest results. Our results are accurate to the second digit number, because we only retain the dominant potential well in each $\lambda$ manifold. The main advantage of our method is that it is simple and physically clear. Because we have analytical expressions for most of our matrix elements, our calculations are simple and fast, whereas the other results we compare with need large scale numerical calculations. We can, therefore, easily to extend to very high lying doubly excited states. Because there is no other data to compare with, we do not report the results here. This method can also be extended to higher L states, with the only added difficulty that analytical expressions for matrix elements are more complicated. Another direction of improvement would be to include more potential wells in each $\lambda$ manifold to yield more accurate eigenvalues. 
Table 5.9. Eigenvalues calculated by equation (5.32) for $\mathrm{He}$ and $H^{-}$of ${ }^{3} \mathrm{P}^{e}$ states, where $\lambda_{\max }=118$.

\begin{tabular}{lllllll} 
& \multicolumn{3}{c}{$\mathrm{He}^{3} P^{e}$} & & \multicolumn{3}{c}{$H^{-3} P^{e}$} \\
${ }_{n}(K, T)_{N}^{A}$ & present & Other[Ref.] & Ref. 97 & present & Ref. 51 & Ref. 97 \\
${ }_{2}(0,1)_{2}^{+}$ & -0.70927 & $-0.71519[82]$ & & -0.12415 & & \\
${ }_{3}(1,1)_{3}^{+}$ & -0.34083 & & -0.33609 & -0.05950 & -0.06376 & -0.06276 \\
${ }_{4}(2,1)_{4}^{+}$ & -0.19628 & & -0.19444 & -0.03459 & -0.03723 & -0.037225 \\
$5(3,1)_{5}^{+}$ & -0.12671 & & -0.12639 & -0.02242 & -0.02458 & -0.024575 \\
$6(4,1)_{6}^{+}$ & -0.08794 & & & -0.01468 & -0.01738 & \\
$7(5,1)_{7}^{+}$ & -0.06394 & & & -0.00985 & & \\
$8(6,1)_{8}^{+}$ & -0.04782 & & & & & \\
$9(7,1)_{9}^{+}$ & -0.03454 & & & & &
\end{tabular}




\section{CHAPTER 6}

\section{CONCLUSIONS AND FUTURE PROSPECTS}

This thesis has presented the first steps in a well defined program for calculating doubly excited states through an analysis of the Schrödinger equation in pair (hyperspherical) coordinates. Diagonalizing the interaction at fixed $\lambda$, the grand angular momentum in the problem, provides potential wells and angular basis functions, described throughout in pair coordinates. In particular, each of these potential wells converges at large $\mathrm{R}$ to the grandparental or double ionization limit. The Pair-Rydberg formula can give fairly good eigenvalues especially for very high doubly excited states, where data is very sparse both theoretically and experimentally. By including the non-diagonal couplings involving the derivatives $\frac{d}{d R}$ and $\frac{d^{2}}{d R^{2}}$, improved results are obtained. If we couple more terms in $\lambda$, the results will be more accurate.

Our method is easy to extend to higher $L$ values, the only difficulty lying in that the analytical expressions for matrix elements are more complicated.

In this thesis work, we have restricted ourselves to energy eigenvalues for a first investigation. Further work would be to examine wave functions, particularly $F(R)$, and use them to calculate matrix elements such as of the dipole operator between ${ }^{1} S^{e}$ and ${ }^{1} P^{o}$ states. Then it will be possible to compare not only the positions of doubly-excited state resonances excited by photoabsorption from the ground state but also the oscillator strengths. Similarly by 
computing matrix elements of $1 / r_{12}$ between the states and underlying singleelectron continua, the autoionization widths of the resonances can be calculated. This method may also be extended to the problems of atoms in electric and magnetic fields. The added problem is to diagonalize the effective charge operator and the external field perturbation in each $\lambda$ manifold. 


\section{REFERENCES}

[1] R. P. Madden and K. Codling, Phys. Rev. Lett. 10, 516 (1963).

[2] M. E. Rudd, Phys. Rev. Lett. 15, 580 (1965), G. J. Schulz, Phys. Rev. Lett. 10, 104 (1963) and Rev. Mod. Phys. 45, 378 (1973).

[3] R. P. Madden and K. Codling, Astrophys. J. 141, 364 (1965).

[4] P. R. Woodruff and J. A. R. Samson, Phys. Rev. A 25, 848 (1982).

[5] D. W. Lindle, T. A. Ferrett, U. Becker, P. H. Kobrin, C. M. Truesdale, H. G. Kerkhoff and D. A. Shirley, Phys. Rev. A 31, 714 (1975), D. W. Lindle, T. A. Ferrett, P. A. Heimann and D. A. Shirley, Phys. Rev. A 36, 2112 (1987).

[6] H. Kossmann, B. Krassig and V. Schmidt, J. Phys. B 21, 1489 (1988).

[7] M. Zubek, G. C. King, and P. M. Rutter, J. Phys. B 21, 3585 (1988), M. Zubek, G. C. King, P. M. Rutter and F. H. Reed Proc. 15th Int. Conf. on Physics of Electronic and Atomic Collisions, Brighton ed $\mathrm{J}$ Geddes, H. B. Gilbody, A. E. Kingston, C. J. Latimer and H. R. J. Walters (Amsterdam:North-Holland), 1 (1987).

[8] P. J. Hicks and J. Comer, J. Phys. B 8, 1866 (1975).

[9] P. Dhez and D. L. Ederer, J. Phys. B 6, L59 (1973).

[10] P. Morin, M. Y. Adam, I. Nenner, J. Delwiche, M. J. Hubin-Frabskin and P. Lablanquie, Nucl. Instrum. Meth. 208, 781 (1983).

[11] M. Zubek, G. C. King, P. M. Rutter and F. H. Reed, J. Phys. B 22, 3411 (1989).

[12] M. Domke, C. Xue, A. Puschmann, T. Mandel, E. Hudson, D. A. Shirley, G. Kaindl, C. H. Greene, H. R. Sadehgpour and H. Petersen, Phys. Rev. Lett. 66, 1306 (1991).

[13] M. Domke, G. Remmers and G. Kaindl, Phys. Rev. Lett. 69, 1171 (1992).

[14] G. J. Schulz, Phys. Rev. Lett. 13, 583 (1964). 
[15] L. Sanche and P. D. Burrow, J Phys. D 12, 1275 (1972),

[16] J. S. Risley, Rev. Sci. Instrum. 43, 95 (1972), J. S. Risley, A. K. Edwards and R. Geballe, Phys. Rev. A 9, 1115 (1974), J. S. Risley and R. Geballe, Phys. Rev. A 10, 2206 (1974).

[17] C. D. Warner, G. C. King, P. Hammond and J. Slevin, J. Phys. B 19, 3297 (1986), C. D. Warner, P. M. Rutter and G. C. King, J. Phys. B 23, $93(1990)$.

[18] S. Cohen et al, Phys. Rev. A 36, 4728 (1987).

[19] J. F. Williams and B. A. Willis, J. Phys. B 7, L61 (1974), J. Phys. B 8, 1641 (1975), J. F. Williams, J. Phys. B 7, L56 (1974), J. Phys. B 9, 1519 (1976),J. Phys. B 21, 1207 (1988).

[20] H. C. Bryant, D. A. Clark, K. B. Butterield, C. A. Frost, H. Sharifian, H. Tootoonchi, J. B. Donahue, P. A. M. Gram, R. W. Hamm, C. J. Pratt, M. A. Yates, and W. W. Smith, Phys. Rev. A 272889 (1983), P. G. Harris, H. C. Bryant, A. H. Mohagheghi, R. A. Reeder, H. Sharifian, C. Y. Tang, H. Tootoonchi, J. B. Donahue, C. R. Quick, D. C. Rislove, W. W. Smith and J. E. Stewart, Phys. Rev. Lett. 65, 309 (1990), P. G. Harris, H. C, Bryant, A. H. Mohagheghi, R. A. Reeder, C. Y. Tang, J. B. Donahue and C. R. Quick, Phys. Rev. A 42, 6443 (1990), M. Halka, H. C. Bryant, E. P. Mackerrow, W. Miller, A. H. Mohagheghi, C. Y. Tang, S. Cohen, J. B. Donahue, A. Hsu, C. R. Quick, J. Tiee and K. Rosaz, Phys. Rev. A 44, 6127 (1991).

[21] J. N. H. Brunt, G. C. King and F. H. Reed, J. Phys. B 9, 2195 (1977).

[22] S. J. Cvejanovic, J. Comer and F. H. Reed, J. Phys. B 7, 468 (1974), S. J. Cvejanovic and F. H. Reed, J. Phys. B 7, 1180 (1974).

[23] R. E. Kennerly, R. J. Van Brunt and A. C. Gallagher, Phys. Rev. A 23, $2430(1981)$.

[24] S. J. Buckman, P. Hammond, F. H. Read and G. C. King, J. Phys. B 16, 4039 (1983), S. J. Buckman and D. S. Newman, J. Phys. B 20, L711(1987) 20, 5807 (1987).

[25] A. B. Montesquieu, P. B. Cattin, A. Gleizes, A. I. Marrakchi, S. Dousson and D. Hitz, J. Phys. B 21, 3387 (1988), P. M. Capelle, D. H. Oza, P. B. Cattin, A. B. Montesquieu, M. Boudjema, A. Gleizes, S. Dousson and D. 
Hitz, J. Phys. B 22, 271 (989), R. Hutton, H. M. Prior, S. Chantrenns, H. M. Chen and D. Schneider, Phys. Rev. A 39, 4902 (1989), F. W. Meyer, D. C. Griffin, C. C. Havener, M. S. Huq, R. A. Phaneuf, J. K. Swenson and N. Stolterfoht, Phys. Rev. Lett. 60, 1821 (1988).

[26] J. W. Cooper, U. Fano and F. Prats, Phys. Rev. Lett. 10, 518 (1963).

[27] P. G. Burke and D. D. McVicar, Adv. Atom. Mole. Phys. 11, 143 (1965).

[28] T. F. O'Malley and S. Geltman, Phys. Rev. A 137, 1344 (1965).

[29] P. L. Altick and E. N. Moore, Phys. Rev. Lett. 15, 100 (1965).

[30] J. H. Macek, J. Phys. B 1, 831 (1968).

[31] C. D. Lin, Phys. Rev. A 10, 1986 (1974), 11, 1692 (1975), Phys. Rev. A 12, 493 (1975) and Phys. Rev. Lett. 35, 1150 (1975).

[32] M. E. Kellman and D. R. Herrick, J. Phys. B 11, L755 (1978), Phys. Rev. A 12, 413 (1975).

[33] F. Iachello and A. R. P. Rau, Phys. Rev. Lett. 47, 501 (1981).

[34] U. Fano, Rep. Prog. Phys. 46, 97 (1983).

[35] A. R. P. Rau, J Physique Coll 43, 211 (1982).

[36] A. R. P. Rau, Electronic and Atomic Collisions ed. J Eichler, I. V. Hertel and N. Stolterfoht (Amsterdam, Elsevier), p. 711 (1984).

[37] A. R. P. Rau, J Phys. B 17, L75 (1984), Nuclear Instruments and Methods in Physics Research B 56/57, 200 (1991).

[38] A. R. P. Rau, Atomic Physics Vol. 9(World Scientific, Singapore), eds. R. S. Van Dyck and E. N. Fortson, 491 (1984).

[39] G. H. Wannier, Phys. Rev. 90, 817 (1953), Phys. Rev. 100, 1180 (1955).

[40] A. R. P. Rau, Phys. Rev. A 4, 207 (1971).

[41] R. Peterkop, J. Phys. B 4, 513 (1971).

[42] U. Fano and A. R. P. Rau, Atomic Collisions and Spectra (Academic, Orlando, 1986), Chapter 10. 
[43] F. H. Read, J. Phys. B 16, L499 (1977), Aust. J. Phys. 35, 475 (1982) and J. Phys. B 23, 951 (1990).

[44] A. R. P. Rau, J. Phys. B 16, L699 (1983).

[45] H. Wang, J. Phys. B 19, 3401 (1986).

[46] Q. Molina, Phys. Rev. A 39, 3298 (1989).

[47] J. M. Feagin and J. Briggs, Phys. Rev. Lett. 57, 984 (1986), Phys. Rev. A 37, 4599 (1988).

[48] J. M. Rost and J. S. Briggs, J. Phys. B 21, L233 (1988), J. Phys. B 22, 3587 (1989).

[49] A. Pathak, A. E. Kingston and K. A. Berrington, J. Phys. B 21, 2939 (1988), A. Pathak, P. G. Burke and K. A. Berrington, J. Phys. B 22, 2759 (1989).

[50] M. Aymar, J. Phys. B 22, 2359 (1989).

[51] Y. K. Ho and J. Callaway, Phys. Rev. A 27, 1887 (1983), J. Phys. B 17, L599 (1984), Phys. Rev. A 34, 130 (1986), J. Phys. B 18, 3481 (1985), Y. K. Ho, Phys. Rev. A 19, 2347 (1979), 23, 2137 (1981), 35, 2035 (1987), Phys. Rev. A 41, 1492 (1990), J. Phys. B 10, L373 (1977), 23, L71 (1990).

[52] Y. Komninos and C. A. Nicolaides, J. Phys. B 19, 1701 (1986), C. A. Nicolaides and Y. K. Komninos, Phys. Rev. A 35, 999 (1987), M. Chrysos, Y. Komninos, Th. Mercouris and C. A. Nicolaides, Phys. Rev. A 42, 2634 (1990).

[53] I. K. Dmitrieva and G. I. Plindov, J. Phys. B 22, 1297 (1989).

[54] E. de Prunele, Phys. Rev. A 45, 2070 (1992).

[55] M. Crance and L. Armstrong Jr., Phys. Rev. A 26, 694 (1982).

[56] A. R. P. Rau, Pramana 23, 297 (1989).

[57] C. D. Lin and S. Watanabe, Phys. Rev. A 35, 4499 (1987).

[58] J. Muller, J. Burgdörfer and D. Noid, Phys. Rev. A 45, 1471 (1992). 
[59] G. Ezra, K. Richter, G. Tanner and D. Wintgen, J. Phys. B 24, L413 (1991).

[60] M. F. Fels and A. U. Hazi, Phys. Ref. A 4, 662 (1970).

[61] A. U. Hazi and H. S. Taylor, Phys. Rev. A 4, 1109 (1970).

[62] J. Callaway and A. R. P. Rau, J. Phys. B 11, L289 (1978).

[63] R. S. Oberoi, J. Phys. B 5, 1120 (1972).

[64] P. G. Burke and H. M. Schey, Phys. Rev. 126, 163 (1962).

[65] P. G. Burke and A. Temkin and J. F. Perkins, Phys. Rev. A 153, 177 (1967), P. G. Burke and A. Temkin, Phys. Rev. A 182, 15 (1969), Phys. Rev. A 8, 21 (1973), A. K. Bhatia, Phys. Rev. A 15, 1315 (1977), A. K. Bhatia, A. K. Bhatia and A. Temkin, Phys. Rev. A 11, 2018 (1975).

[66] J. Callaway and R. W. Labahn, Phys. Rev. 168, 12 (1968).

[67] D. R. Herrick and O. Sinanoglu, Phys. Rev. A 11, 97 (1975).

[68] K. T. Chung and J. C. Y. Chen, Phys. Rev. A 13, 1655 (1976), K. T. Chung, Phys. Rev. A 6, 1809 (1972).

[69] J. Callaway, Phys. Rep. 45, 91 (1978), J. Callaway, Phys. Lett. 68A, 315 (1978), 81 A, 495 (1981), J. Callaway and J. W. Wooten, Phys. Lett. 45A, 85 (1973), J. Callaway, R. S. Oberoi and G. J. Seiler, Phys. Lett. 31A, 547 (1970), J. Callaway, Phys.Rev. A 26, 199 (1982), 37, 3692 (1988), S. Wakid and J. Callaway, Phys. Lett. 78A, 137 (1980).

[70] D. H. Oza, Phys. Rev. A 33, 824 (1986).

[71] A. R. P. Rau and Q. Molina, J. Phys. B 22, 189 (1987).

[72] A. R. P. Rau, in Aspects of Electron-Molecule Scattering and Photoionization, A. I. P. Conf. Proc. 204, ed. by Herzenberg(Am. Inst. of Physcics, New York, 1990), p. 24.

[73] D. R. Herrick, Adv. Chem. Phys. 52, 1 (1983).

[74] C. D. Lin, Adv. Mol. Phys. 22, 77 (1986), Z. Chen and C. D. Lin, Phys. Rev. A 42, 18 (1990). 
[75] I. C. Percival, Proc. Roy. Soc(London) A 353, 289 (1977).

[76] D. R. Herrick, Phys. Rev. A 12, 413 (1975).

[77] C. D. Lin and J. H. Macek, Phys. Rev. A 29, 2317 (1984).

[78] C. D. Lin, Phys. Rev. Lett. 51, 1384 (1984) and Phys. Rev. A 29, 1019 (1984).

[79] L. Zhang and A. R. P. Rau, Phys. Rev. A, 46, 6933 (1992).

[80] L. Zhang and A. R. P. Rau, in preparation.

[81] H. Fukuda, N. Koyama and M. Matsuzawa, J. Phys. B 20, 2959 (1987), N. Koyama, A. Takofuji and M. Matsuzawa, J. Phys. B19, L331 (1986), 22, 553 (1989).

[82] H. R. Sadeghpour and C. H. Greene, Phys. Rev. Lett. 65, 313 (1990), Phys. Rev. A 39, 115 (1989) and H. R. Sadeghpour, Phys. Rev. A 43, 5821 (1991).

[83] H. Klar and M. Klar, J. Phys. B 13, 1057 (1980).

[84] A. R. P. Rau and L. Zhang, Phys. Rev. A 42, 6342 (1990).

[85] H. Feshbach, Ann. Phys. (New York) 5, 357 (1958), 19, 287 (1962).

[86] P. G. Burke and H. M. Schey, Phys. Rev. 126, 147 (1962), P. G. Burke, H. M. Schey and K. Smith, Phys. Rev. 129, 1258 (1963), P. G. Burke, S. Ormonde and W. Whitaker, Proc. Phys. Soc. Lond. 92, 319 (1967).

[87] J. Callaway and J. F. Williams, Phys. Rev. A 12, 2312 (1975), J. Callaway and M. R. C. McDowell and L. A. Morgan, J. Phys. B 8, 2181 (1975), 9, 2043 (1976), J. Callaway amd J. W. Wooten, Phys. Rev. A 11, 1118 (1975), 9, 1924 (1974), J. Callaway and D. H. Oza, Phys. Rev. A 32, 2682 (1985).

[88] R. K. Nesbet, Phys. Rev. 179, 60 (1969).

[89] J. Aguilar and J. M. Combes, Commun. Math. Phys. 22, 269 (1971).

[90] E. Balslev. and J. M. Combes, Commun. Math. Phys. 22, 280 (1971).

[91] B. Simon, Commun. Math. Phys. 27, 1 (1972). 
[93] I. I. Sobel'man, Introduction to the Theory of Atomic Spectra, Pergamon Press, ed. T. Haar (Oxford · New York - Toronto - Sydney · Braunschweig 1972).

[94] M. Rotenberg, R. Bivins, N. Metropolis and J. K. Wooten Jr, 3-j and 6-j Symbol, The Technology Press (Massacgusetts Institute of Technology, Cambridge Mass., 1959).

[95] Handbook of Mathematical Functions, ed. by M. Abramowitz and I. A. System(Dover, New York, 1965), Chapters 15 and 22.

[96] U. Fano, F. Robicheaux and A. R. P. Rau, Phys. Rev. A 37, 3663(1988).

[97] Y. K. Ho, J. Phys. B 15, L691 (1982), Phys. Rev. A 27, 1887 (1983), Phys. Rev. A 34, 130 (1986), Z. Phys. D. 21, 191 (1991), Chinese Journal of Physics 29, 327 (1991), Phys. Rev. A 45, 148 (1992), Y. K. Ho and A. K. Bhatia, Phys. Rev. A, in press (1993).

[98] See, for instance, Atomic Excitation and Recombination in External Fields, edited by M. H. Nayfeh and C. W. Clark(Gordon and Breach, New York, 1985); H. Friedrich and D. Wintgen, Phys. Rep. 183, 37 (1989); Atomic Spectra and Collisions in External Fields, edited by K. T. Taylor, C. W. Clark, and M. H. Nayfeh(Plenum, New York, 1988).

[99] A. Hölle, J. Main, G. Wiebusch, H. Rottke, and K. H. Welge, Phys. Rev. Lett. 61, 161(1988), and references therein.

[100] A. R. P. Rau, Phys. Rev. Lett. 63, 244 (1989).

[101] A. R. P. Rau, Rep. Prog. Phys. 53, 181 (1990).

[102] A. Sommerfeld, Mechanics (Academic, New York, 1952), Sec. IV. 26.

[103] H. A. Kramers and G. P. Ittmann, Z. Phys. 53, 533 (1929), 58, 217 (1929), 60, 663 (1930).

[104] S. C. Wang, Phys. Rev. 34, 243 (1929); O. Klein, Z. Phys. 58, 730 (1929).

[105] C. H. Townes and A. L. Schawlow, Microwave Spectroscopy (Dover, New York, 1975), Chap. 4. 
[106] W. G. Harter, J. Chem. Phys. 85, 5560 (1986); Comput. Phys. Rep. 8, 319 (1988); W. G. Harter and C. W. Patterson, J. Chem. Phys. 80, 424 (1984).

[107] T. Uzer, Phys. Rev. A 42, 5787 (1990). The reduction of anharmonicoscillator Hamiltonians into an asymmetric rotor has also been considered in D. K. Saham, S. W. McWhorter, and T. Uzer, J. Chem. Phys. 91,219 (1989).

[108] L. D. Landau and E. M. Lifshitz, Quantum Mechanics: Non-Relativistic Theory(Pergamon, Oxford, 1977), Sec. 77.

[109] M. Baranger and K. Kumar, Nucl. Phys. 62, 113 (1965).

[110] P. A. Braun, Zh. Eksp. Teor. Phys. 84, 850 (1983) [ Sov. Phys.- JETP 57, $492(1983)]$.

[111] E. A. Soloviev, Pis'ma Zh. Eksp. Teor. Fiz. 34, 278 (1981) [JETP Lett. 34, $265(1981)]$.

[112] E. A. Soloviev, Zh. Eksp. Teor. Fiz. 89, 1991 (1985) [Sov. Phys. - JETP 62, $1148(1985)]$.

[113] R. A. Pullen and A. R. Edmonds, J. Phys. A 73, L477 (1981).

[114] J. L. Anchell, J. Chem. Phys. 92, 4342 (1990).

[115] See, for instance, Chaotic Behavior in Quantum System, edited by G. Casati (Plenum, New York, 1985).

[116] L. D. Landau and E. M. Lifshitz, Ref. 74, Sec. 103.

[117] Our terminology differs slightly from the standard one for the asymmetric rotor. The $(x, y, z)$ axes in our usage are laboratory-fixed axes, with $z$ being chosen to conform to the usual quantization axis. The reason for our choice, and for $M$ as the usual projection of angular momentum along this axis, is to faciliate the correspondences in Sec. A.4 between $M$ and $l$, and $J$ and $n$. An asymmetric rotor is usually described as in (A.17) but with body-fixed axes $\xi, \eta, \zeta$ in place of $(\mathrm{x}, \mathrm{y}, \mathrm{z})$. Owing to the complete analogy between the two Hamiltonians, the results are formally identicalsee, especially, Problem 6 in Ref. 85. As per that discussion, (A.17) may be viewed either as an asymmetric rotor in Sec.A.3 B would be phrased in terms of $J_{\xi}^{2}$ instead of the $J_{x}^{2}$ (or $J_{y}^{2}$ ) that we have chosen. The quantum 
numbers $K, M$, and $J$ would then correspond to the mutually commuting operators $J_{\xi}, J_{z}$, and $J^{2}$, respectively. If the difference in language is kept in mind, no confusion should arise as is clear from the discussion in Ref. 85 .

[118] G. W. King, J. Chem. Phys. 15, 820 (1947).

[119] L. D. Landau and E. M. Lifshitz, Ref. 74, Sec. 37.

[120] For semiclassical approximations and the connection to coherent states, see J. Kurchan, P. Lebouef, and M. Saraceno, Phys. Rev. A 40, 6800 (1989), and references therein. 


\section{APPENDIX A}

\section{MAPPING DEGENERATE PERTURBATIONS IN ATOMS ONTO AN ASYMMETRIC ROTOR}

\section{A.1 Introduction}

In previous chapters, we have focused on doubly excited ridge states. As we discussed in chapters 4 and 5 , the diagonalization in the grand angular momentum $\boldsymbol{\Lambda}^{2}$ manifolds will lead us to get eigenvalues $C_{\lambda Q}$ and eigenvectors as shown in Fig. 7 and 8,9,10 and 11. The plots in Fig. 7 are similar to other diagonalization problems in atomic physics, for example, atoms in electric field or magnetic field where the perturbations mix degenerate states.

There has been considerable interest in very nonperturbative problems in atomic structure and dynamics in recent years. Thus the application of external, laboratory strength fields, whether electric or magnetic, leads to complicated phenomena if the atom is highly excited (a Rydberg state, even in a simple atom such as hydrogen) and the external fields then no longer are merely perturbative [98-99]. In two or more electron atoms, doubly excited (or multiply excited) states again present situations wherein the electron-electron interaction is not just perturbative and amenable to treatment as a mean field but leads to strong correlation effects and, therefore, to qualitatively unexpected phenomena as discussed in earlier chapters [34,42]. All these are problems in which the interaction is not weakly perturbative and, at the same time, the full Hamiltonian is not separable in any coordinate system. Therefore new 
techniques and insights are called for in understanding the rather rich phenomena that have been observed in these systems. Common elements have come to be recognized of these diverse systems, which at first sight appear to be independent [100-101].

The understanding of common features is carried a step further here by mapping diverse problems onto an asymmetric rotor. The study of this rotor has a long history, both in classical [102] and quantum physics [103104]. Its importance has also continually remained in the forefront of the field of molecular spectroscopy [105-106] while receding to the background in the study of atomic systems. But we wish to point out its direct relevance to the atomic phenomena in the above paragraph and to many others [107]. Indeed, there is a one-to-one correspondence of coordinates, quantum numbers, and the structure of the spectrum between the asymmetric rotor and the many physical problems. The angular momentum quantum numbers $J$ and $M$ of the rotor map onto the principal $(n)$ and orbital momentum $(l)$ quantum numbers, respectively, of the atomic problems. Indeed, a primary conclusion of ours is that (i) degenerate manifolds $\{n l\}$ in atomic and nuclear problems behave exactly like an angular momentum space, and (ii) several quadratic interactions that lift the degeneracy by mixing the different $\mid n l>$ states may be viewed on the common footing of an asymmetric rotor whose eigenstates involve an analogous mixing of $\mid J M>$ states.

In the classical limit, which obtains at high $J$, the asymmetric rotor has two stable motions associated with the axes corresponding to maximum and minimum moments of inertia. Rotation with respect to the intermediate third axis is unstable [102]. The existence of two classes of localized states at high excitation (large $\mathrm{n}$ ) in many problems maps into the two stable motions 
of the rotor. The few states in an in-between "separatrix" region are identified with the third unstable motion. The localization of quantum states has come to be recognized as chiefly responsible for very sharp spectral features at high excitation. It now finds a natural explanation in terms of a rotor's motion about specific axes.

The Hamiltonian of a general, asymmetric rotor [102,105] is $A J_{x}^{2}+$ $B J_{y}^{2}+C J_{z}^{2}$. Its diagonalization in a $\mid J M>$ basis involves tridiagonal matrices or a three-term difference equation. It has been emphasized [100] that such structure is characteristic of many problems in atomic and nuclear physics wherein a quadratic Hamiltonian mixes degenerate $l$ states of an $n$ manifold. When $n$ is large, the difference equation can be cast $[96,100]$ in terms of the spheroidal differential equation in a continuous variable $u=\left(l+\frac{1}{2}\right) / n$. In this work, we point out that the matrix elements of the asymmetric rotor are also of exactly the same structure in the variable $M /\left(J+\frac{1}{2}\right)$ and therefore, that the asymmetric rotor can also be cast in terms of the same universal, spheroidal equation. All the consequences that follow from the spheroidal structure - in particular, that most states fall into two classes, characterized by localized eigenvectors with a characteristic exponential falloff in $\left(-n u^{2}\right)-$ are, therefore, common to these problem. The two classes are known to be linked by a conjugation transformation [96,100], which is now recognized as an interchange of the axes with maximum and minimum moments of inertia of the rotor. The spheroidal equation is characterized by three constants that play the role of the three coefficients A, B and C of the rotor Hamiltonian. In the next section we present a table which gathers together in one place all the varied problems, and thereby facilitates the viewing of all them on a single footing. 
The arrangement of this chapter is as follows. Section A.2 briefly summarizes the various problems that have been considered involving diagonalization in degenerate manifolds, presenting the matrix elements involved in these tridiagonal problems. Section A.3 opens with a simple pedagogical example, which was indeed the one that led to an development, namely, diagonalization of the component $J_{x}$ in a $\mid J M>$ basis. This almost trivial tridiagonal problem not only leads naturally to the next step, the operator $J_{x}^{2}$, which has the same eigenvectors and which provides the entry into the more general asymmetric rotor, but it is, in fact, an intimate rendering of an actual physical problem, namely, the Stark effect in excited states of hydrogen [108]. Section A.3 then proceeds to the complete asymmetric rotor problem, recording matrix elements, eigenvalues, and eigenvectors together with numerical illustrations for a high value of $J$. Section A.4 provides the mapping of the problems in Section A.2 into the asymmetric rotor, with the diamagnetic effect, doubly excited states, and two-nucleon quadrupole interaction $[100,108]$ as illustrative examples. Section A.5 gives a brief summary of the results.

\section{A.2 Tridiagonal Matrices For Varied Problems}

A.2.1 General Structure The interactions we consider gener-

ally have a radial and an angular factor as, for instance, the diamagnetic interaction that is proportional to $x^{2}+y^{2}=r^{2} \sin ^{2} \theta$. The former is the dynamical part, the latter the geometrical. In a spherical basis $\mid n l m>$ of the base problem, selection rules stemming from the angular part connect a state in the degenerate manifold only to its nearest neighbors. Thus, in the above example, $l$ is connected to $l \pm 2$ (besides the diagonal component), in other problems, 
$l$ to $l \pm 1$. Thereby the action of the interaction within such degenerate manifolds is analyzed in terms of a three-term difference equation $[96,100,109]$ for the eigenvalues $\mathrm{E}$ and corresponding eigenvector coefficients $\left\{a_{l}\right\}$,

$$
W_{l-1} a_{l-2}+V_{l} a_{l}+W_{l+1} a_{l+2}=E a_{l}
$$

In the problems we consider below, the matrix elements are quadratic functions of the variable $u=\left(l+\frac{1}{2}\right) / n$ with a common structure (more on its significance below)

$$
W_{l+1 / 2}=\omega\left(1-u^{2}\right), \quad V_{l}=v_{0}-v_{1} u^{2}
$$

where the triplet $\omega, v_{0}$ and $v_{1}$ characterizes the interaction. Our interest in high excitation for all these problems means that $n$ is large, and the set of points $u=\left(l+\frac{1}{2}\right) / n$, even though discretely distributed in the interval $(0,1)$, is dense, permitting $u$ to be regarded as a continuous one-dimensional coordinate in this interval. A simple Taylor expansion of each term in (A.1) permits then a recasting of it into the differential equation $[96,100]$

$$
\left[\left(1-u^{2}\right) a^{\prime}(u)\right]^{\prime}+\left(n^{2} / 4\right)\left[2\left(1-u^{2}\right)+\left(v_{0}-E-v_{1} u^{2}\right) / \omega\right] a(u)=0
$$

where primes denote differentiation with respect to the argument, here $u$. This is a spheroidal differential equation [95]. Problems involving $a_{l \pm 1}$ instead of $a_{l \pm 2}$ in (A.1) have a coefficient $n^{2}$ in place $n^{2} / 4$ in (A.3). In all cases, since $n^{2}$ is large, asymptotic formulas for the spheroidal equation [95] can be used to give simple analytical expressions for eigenvalues and eigenvectors $[96,100]$. As has been discussed, the eigenvalues span a finite range and are equally spaced at either end [only in the special case when the coefficient of $u^{2} a(u)$ in (A.3) vanishes does the equation reduce to that of Legendre polynomials with a pure 
rotor spectrum]. The two ends are related by a conjugation transformation, which amounts to switching the sign of the off-diagonal matrix elements, that is, $\omega \rightarrow-\omega$. In terms of the eigenvectors, this is equivalent to a local gauge transformation where alternate entries in $\left\{a_{l}\right\}$ are changed in sign. We will now list the values of $\omega, v_{0}$, and $v_{1}$ for varied physical problems.

\section{A.2.2 Linear Stark Effect in Hydrogen The interaction eEz} due to an external, static electric field $\mathbf{E}$ along the $\mathrm{z}$ direction, or its standard scaled dimensionless form $z / n^{2} a_{0}$, mixes states $l \rightarrow l \pm 1$ and has no diagonal coupling: $v_{0}=v_{1}=0$. This is a particularly simple case, where the last term in (A.3) reduces to a constant $-\mathbf{E} / \omega$. The eigenvalues are constantly spaced throughout with conjugate ones differing only in sign, and conjugate eigenvectors differing in an alternating sign change of the coefficients $\left\{a_{l}\right\}$. The extreme states are localized at $\theta=0$ and $\pi$, that is, along and against the field. With no diagonal $\mathrm{V}$ and only off-diagonal $\mathrm{W}$ so that $\omega$ becomes a mere scale factor, this problem is one of pure geometry, that of the operator $\cos \theta$. This observation and that the eigenvalues lie equally spaced between -1 and 1 , symmetrically on either side of 0 , will become even more self-evident when we link this problem in Sec. A. 3.1 to the diagonalization of $J_{x}$.

A.2.3 Diamagnetic Effect in Hydrogen The interaction $\left(e^{2} B^{2} / 8 m c^{2}\right)\left(x^{2}+y^{2}\right)$ is, apart from a multiplicative constant, $\frac{1}{2}\left(r^{2} / n^{4} a_{0}^{2}\right) \sin ^{2} \theta$ in dimensionless form. Referring to [96] for details, Table A.1 lists the values of $\omega, v_{0}$, and $v_{1}$ for this and other problems. Analytical expressions for eigenvalues and eigenvectors, together with a comparison with numerical results, have been presented before. Table A.2 records one here as a sample of such 
Table A.1. Structure of matrix clements, eigenvalues, and asymmetry parameter of the asymmetric rotor, for various interactions. The matrix elements are quadratic functions of $u$ is $M /(J+1 / 2)$ for the asymmetric rotor and $(l+1 / 2) / n$ for the other system. Yalues of the three coefficients involved $v_{0}, v_{1}$ and $\omega$ are shown. In the columns under "Eigenvalues" the extreme values spanned by them as well as an intermediate one that marks the separatrix are shown. For details on the asymmetric rotor, sec Sec. A. 3, and for all the other systems, see Sec. 2 .

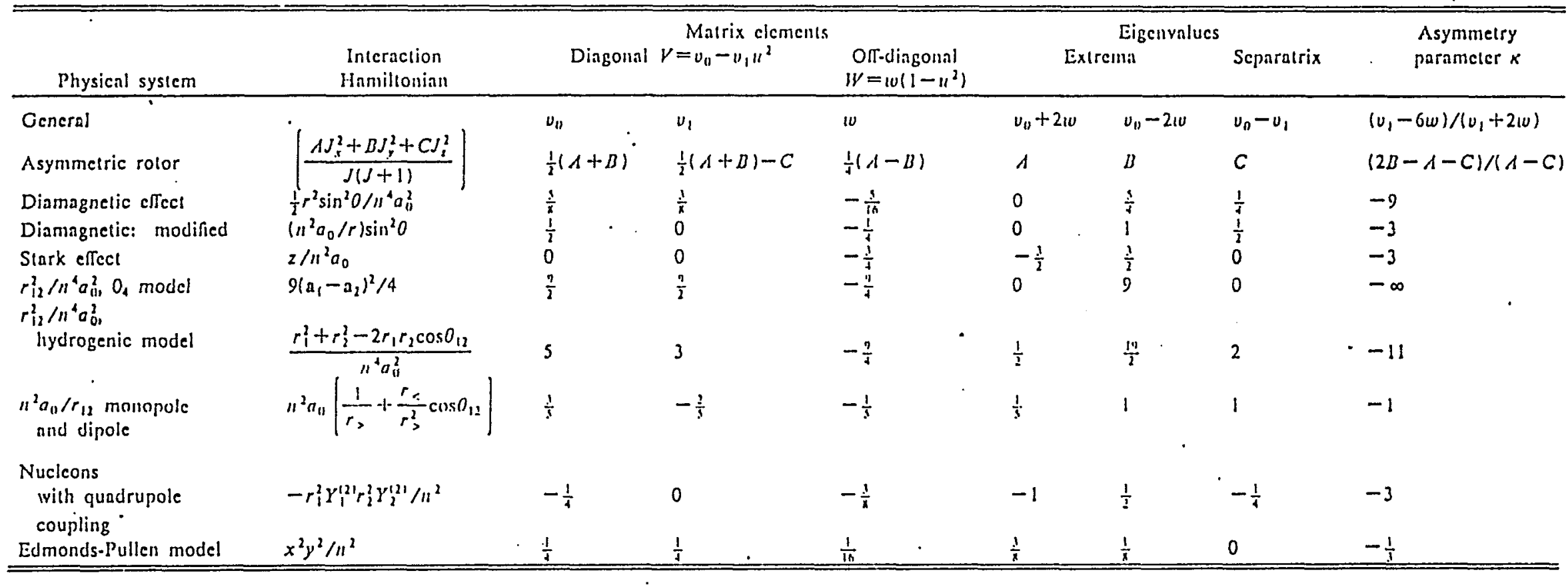


calculations. The eigenvalues stretch over the interval $\left(0, \frac{5}{4}\right)$, divided into two groups by a separatrix at $\frac{1}{4}$. The span $\left(0, \frac{1}{4}\right)$ has been referred to in the literature $[98-99,101]$ as vibrator states and has wave functions localized around $\theta=0$ and $\pi$, that is, along the field. The conjugate span $\left(\frac{1}{4}, \frac{5}{4}\right)$, called rotor states, is localized around $\theta=\frac{\pi}{2}$, perpendicular to the field.

Our treatment emphasizes the common features of these two spans and indeed that they are conjugates in a well-defined sense. In both cases, the eigenvalues are equally spaced starting from the extreme values 0 and $\frac{5}{4}$. Note in Table A.2 the near degeneracy (a small splitting is exhibited only on going to more significant figures) of odd- and even-parity states in the $\left(0, \frac{1}{4}\right)$ span. This reflects their localization at $\theta=0$ and $\pi$, these two directions being connected by the parity transformation $z \rightarrow-z$. In the $\operatorname{span}\left(\frac{5}{4}, \frac{1}{4}\right)$ with localization at $\theta=\frac{\pi}{2}$, the alternating even- and odd-parity states together form an equally spaced family much as in a one-dimensional oscillator. Note also for the eigenvectors a close similarity, apart from an alternating sign, of $a_{l}$ for the extreme eigenvectors as displayed in Fig. 4 of Ref 95.

For the diamagnetic effect on high Rydberg states, much attention has been given to the role that the groups of states play in the near-zeroenergy structures seen in this system [98-99]. In this work, we are, however, not concerned specifically with these aspects, nor with the classically unstable periodic orbits that have been computed and their connection in a nonintegrable system like this with the observed quantal spectrum. Our aim instead is to understand better the two groups of states themselves and see generic aspects of the very existence of such states in a wide variety of problems. We do not directly face questions of integrability because of our focus on a single $n$ manifold. In understanding the full physical system, one will have to look, of 
course, at the subsequent mixing of states from different $n$. What is noteworthy is that the existence of two groups of localized states is already evident at fixed $n$ and does not seem to be completely vitiated in the subsequent mixing across in $n$. This latter part still remains to be fully explained and in that explanation will lie the resolution of puzzles associated with nonintegrability.

The separatrix at $\frac{1}{4}$ between the two groups of states has been analyzed on semiclassical grounds in terms of a cone of semiangle $\theta=\arcsin 1 / \sqrt{5}$ (or, equivalently, $\cot ^{-1} 2$ ), which divides the Kepler ellipses of the hydrogenic orbits into two groups, those in which the tip of the semimajor axis precesses inside the cone about the longitudinal direction with respect to the field, and those in which the tip is confined to lie outside this cone and, therefore, grouped around $\theta=\pi / 2$ perpendicular to the field [111]. The role played by dynamics and geometry becomes even clearer now through our capsuled entries in Table A.1. The numbers 0 and $\frac{5}{4}$ are simply $V \pm 2 W$ for $u \simeq 0$ (small $l$ ), that is, $v_{0} \pm 2 \omega$, whereas $\frac{1}{4}$ pertains similarly to $v \simeq 1$ (large $l$ ), that is, $v_{0}-v_{1}$ (since $\mathrm{W}$ vanishes in this limit, the two conjugate combinations coincide). The large- $l$ values are contained only in the separatrix, not in most of the other eigenvectors which constitute the two groups of localized states. The difference in $\mathrm{V}$ for small and large $l$ is the result of the radial matrix element of $r^{2}$. Had this been a constant for all $l$ as, for instance, it would have been had we considered the operator $\left(\sin ^{2} \theta\right) / r$ instead of $\frac{1}{2} r^{2} \sin ^{2} \theta$, then such a model problem would have had the entries shown in Table A.1 for the row "diamagnetic modified." In such a case, the two spans of eigenvalues would be $\left(0, \frac{1}{2}\right)$ and $\left(\frac{1}{2}, 1\right)$ with the separatrix exactly in the middle at $\theta=\arcsin 1 / \sqrt{2}=\pi / 4$. These numbers are, of course, in natural correspondence with the extreme and middle values 
of $\theta$ and $\sin ^{2} \theta$. We can, therefore, regard the radial dynamics as distorting the separatrix cone from the geometrically symmetric $45^{\circ}$ to $\arcsin 1 / \sqrt{5}$.

\section{A.2.4 Electron-electron Interaction in Doubly Excited States}

Although this is a two-particle problem unlike the two previous one-particle ones, the physics and mathematics are closely in parallel [72]. The localizations now are not in an angle $\theta$ with respect to an external field direction but in the angle $\theta_{12}$ between the two radial vectors $\mathbf{r}_{1}$ and $\mathbf{r}_{2}$. The mixing of degenerate manifolds of states of the same ${ }^{2 S+1} L_{J}^{\pi}$ symmetry formed with both electrons in a high-hydrogenic manifold $n$ has been of interest for describing angular correlations between electrons in high doubly excited states [35,71]. As it stands, the diagonalization of $e^{2} / r_{12}$ in such a manifold presents a more general matrix than one of tridiagonal form. Approximate models of this interaction have, however, been quite successful [101]. Thus several models that consider the operator $r_{12}^{2}$ instead of the $1 / r_{12}$ operator lead to tridiagonalization because $r_{12}^{2}=r_{1}^{2}+r_{2}^{2}-2 r_{1} r_{2} \cos \theta_{12}$ couples $l$ only with itself or with $l \pm 1$. Alternatively, if a multipole expansion is made of $e^{2} / r_{12}$, the leading two terms are isotropic ("monopole") and of "dipole" form $P_{1}\left(\cos \theta_{12}\right)$; once again, the angular selection rules lead only to nearest-neighbor coupling. The well-know success of the multipole expansion in atomic physics and that these two are the dominant pieces of the interaction makes such an approximation valid. Table A.1 records the relevant coefficients of the interaction in ${ }^{1} S$ states for several such models. The first is for $\left(r_{12}^{2} / n^{4} a_{0}\right)$ written as the square of the difference between the Runge-Lenz vectors a for the two electrons [101,112]. The second [101] is for $r_{12}^{2}$ written as above with hydrogenic expressions for $\left\langle r_{1}^{2}\right\rangle$, etc. The third is for the monopole and dipole expansion terms of $n^{2} a_{0} / r_{12}$ with 
Table A.2. Eigenvalues of the diamagnetic interaction $E_{D}=\frac{1}{2} r^{2} \sin ^{2} \theta /\left(n^{4} a_{0}^{2}\right)$, for $n=51$ and $m=0$. The even- and odd-parity states form two separate systems; note their degeneracy below the separatrix at 0.25 and an interlacing above that.

\begin{tabular}{llll}
\hline \hline Even & Odd & Even & Odd \\
\hline 0.02173 & 0.02173 & 0.4375 & \\
0.06321 & 0.06321 & 0.4840 & 0.4603 \\
& & & 0.5086 \\
0.1022 & 0.1022 & 0.5340 & 0.5604 \\
0.1386 & 0.1386 & 0.5877 & 0.6159 \\
0.1722 & 0.1722 & 0.6499 & \\
0.2026 & 0.2026 & 0.7056 & 0.6748 \\
0.2289 & 0.2292 & 0.7699 & 0.7373 \\
& & & 0.8033 \\
0.2479 & 0.2523 & 0.8376 & 0.8727 \\
0.2646 & & 0.9088 & \\
& 0.2763 & & 0.9457 \\
0.2899 & & 0.9835 & \\
& 0.3047 & & 1.0222 \\
0.3207 & & 1.0617 & \\
& 0.3377 & & 1.1020 \\
0.3557 & 0.3747 & 1.1433 & 1.1854 \\
0.3947 & & 1.2284 & \\
\hline \hline & 0.4156 & & \\
\hline \hline
\end{tabular}


the analytical approximations $\left\langle(n l)^{2}\left|1 / r_{>}\right|(n l)^{2}\right\rangle=\left(3+u^{2}\right) /\left(5 n^{2} a_{0}\right)$ and $<(n l)^{2}\left|r_{<} / r_{>}^{2}\right|(n l)^{2}>=2\left(1-u^{2}\right)^{1 / 2} /\left(5 n^{2} a_{0}\right)$ to numerically calculated values [101]. Here, and in the Sark effect, when $W$ in (A.2) involves $\left(1-u^{2}\right)$ under a square root, the coefficient $n^{2} / 4$ in (A.3) is replaced by $2 n^{2}$, with $u$ in that equation standing for $\left(l+\frac{1}{2}\right) / r \sqrt{2}$.

\section{A.2.5 The Edmonds-Pullen Model A particle moving in the} two-dimensional potential $V(x, y)=\frac{1}{2}\left(x^{2}+y^{2}\right)+\alpha^{2} x^{2} y^{2}$ is another nonseparable problem [113] sharing many of the characteristics of the others discussed in this chapter. Such model problems based on an underlying harmonic-oscillator basis, which has large degeneracies as in Coulomb problems, have been of interest in the study of chaos in dynamical systems [114-115]. Table A.1 records the matrix elements of $x^{2} y^{2} / n^{2}$. The two classes of states [100] now are associated with localization along the $\mathrm{x}$ and $\mathrm{y}$ axes or along the $45^{\circ}$ lines $x= \pm y$.

\section{A.2.6 Two-nucleons with Quadrupole Coupling A realistic}

problem in nuclear physics is the two-particle analog of the Edmonds-Pullen model. With two nucleons in the nth shell in a shell-model basis potential $\frac{1}{2}\left(r_{1}^{2}+r_{2}^{2}\right)$, the dominant nuclear coupling of the quadrupoles gives the interaction $[100,109]-r_{1}^{2} Y_{1}^{(2)} r_{2}^{2} Y_{2}^{(2)} / n^{2}$, once again put in scaled, dimensionless form; the $Y^{(2)}$ are second-rank spherical tensors. The coefficients of the tridiagonal matrix are shown in Table A.1. Fig. 20 gives a sample of the results for eigenvalues and eigenvectors of ${ }^{1} S^{e}$ symmetry. The former lie between -1 and $\frac{1}{2}$, with equal spacing at both ends, in quantitative agreement also with the analytical result $3 \sqrt{2} / n$ for this spacing from the spheroidal equation [100]. Note again the characteristic structure of the extreme eigenvectors and the separatrix. Although this is a concrete problem in nuclear physics, the results 
reduce essentially to pure geometry because of a peculiar circumstance. The radial part of the interaction involves $r^{2}$ for each nucleon. Since this is also the operator that defines the shell-model basis, the diagonal matrix elements are independent of $l$, that is, $v_{1}=0$. Therefore the separatrix at $v_{0}(=-1 / 4)$ lies symmetrically between the extreme values $v_{0} \pm 2 \omega\left(=\frac{1}{2},-1\right)$ and the quadrupolequadrupole interaction in an $n$ manifold reduces to the purely geometrical operator $-P_{2}\left(\cos \theta_{12}\right)=-\left(3 \cos ^{2} \theta_{12}-1\right) / 2$. The extreme values are for $\theta_{12}=0$, $\pi$, and $\pi / 2$ and the separatrix lies in the middle at $\theta_{12}=\pi / 4,3 \pi / 4$. These results parallel exactly the model problem "diamagnetic-modified" where, similarly, an $1 / r$ dependence reduces that atomic problem to one of pure geometry just as the harmonic $r^{2}$ dependence has in this nuclear problem.

A.2.7 Summary To summarize this section, the various problems listed have a common tridiagonal structure given in (A.1) and (A.2), with the values $v_{0}, v_{1}$, and $\omega$ gathered together in Table A.1. Through the connection to the spheroidal equation in (A.3), analytical structures of eigenvalues and eigenvectors can be unraveled, the localization of the states can be associated with specific angular directions, and the role of dynamics and geometry that underlie these results can be spelled out. In Sec. 4, even further insights into these results are developed by mapping all the problems into a common one, the asymmetric rotor. We turn next to a study of the rotor.

\section{A.3 The Asymmetric Rotor at High J}

The asymmetric rotor Hamiltonian $A J_{x}^{2}+B J_{y}^{2}+C J_{z}^{2}$ has been much studied in classical mechanics [102] and quantum mechanics as well, from the earliest days [103-104]. Previous work on the diamagnetic effect in hydrogen 

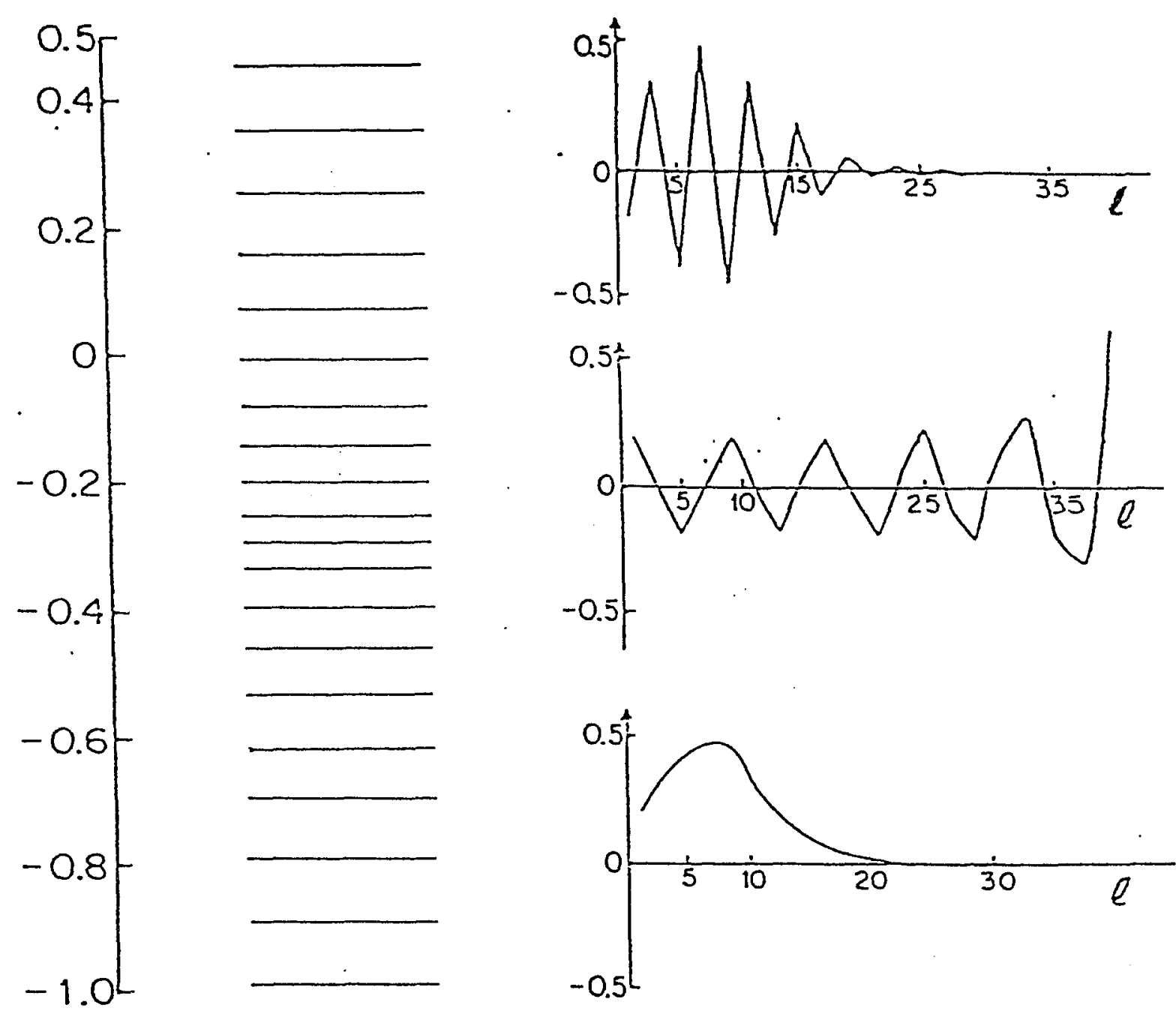

Figure 20. Eigenvalues $E_{Q}$ and representative eigenvectors $\left\{a_{l}\right\}$ of the quadrupole-quadrupole interaction between two nucleons in the shell $n=39$ and coupled to ${ }^{1} S^{e}$ symmetry. The eigenvalues are equally spaced from -1 up and from $\frac{1}{2}$ down, the separatrix lying midway in between at $-\frac{1}{4}$. The distribution in $l$ of the extreme eigenvectors (which, among themselves, are identical except for an alternating sign) is contrasted with that of the separatrix; only the latter involves the high- $l$ values $l \gg n^{1 / 2}$. 
had also specifically associated that problem with the asymmetric rotor and pointed out that the two classes of localized states are as in the asymmetric rotor's localization around the axes with minimum and maximum moments of inertia [96]. The very intimate correspondence between the two problems had, however, not been fully appreciated [107]. Indeed every one of the problems in Sec. A.2 can be mapped one to one onto an asymmetric rotor, the triplet $\left(v_{0}, v_{1}, \omega\right)$ being in direct correspondence with the triplet $(A, B, C)$. Furthermore, the separatrix is associated with the intermediate axis of the rotor, a result that has again not been fully appreciated so far. In this section, the asymmetric rotor is set up as a tridiagonal matrix so as to establish the correspondence with Sec. A.2. But first we begin with a very simple problem, namely, the symmetric rotor with $B=C=0$. This study of $J_{x}^{2}$ is, of course, equivalent to the study of the operator $J_{x}$ because they share the same eigenvectors. The study of $J_{x}$ along the lines of Sec. A.2 and its spheroidal equation affords probably the simplest illustration of this method of handling tridiagonal problem and also has independent pedagogic value.

A.3.1 Diagonalization of $J_{x}$ In the $\mid J M>$ basis, the only nonzero matrix elements of $J_{x}$ are

$$
<J M\left|J_{x}\right| J M \pm 1>=\frac{1}{2}[(J \mp M)(J \pm M+1)]^{1 / 2} .
$$

Diagonalization, therefore, amounts to a difference equation, as in (A.2), but with $V=0$. For large $J$, the matrix elements in (A.4) may be approximated for $M<<J$ as $\frac{1}{2}\left(J+\frac{1}{2}\right)\left(1-x^{2}\right)$ with $x=M\left[\sqrt{2}\left(J+\frac{1}{2}\right)\right]^{-1}$, and the eigenvalue problem reduced to the spheroidal differential equation

$$
\left[\left(1-x^{2}\right) a^{\prime}(x)\right]^{\prime}+\left[4\left(J+\frac{1}{2}\right)^{2}\left(1-x^{2}\right) \pm 4\left(J+\frac{1}{2}\right) E\right] a(x)=0,
$$


which is of the same form as (A.3). The conjugation of switching the sign of the off-diagonal matrix elements leads to the plus-minus sign in the above equation. An asymptotic formula [95] for this prolate spheroidal equation gives for large J,

$$
\pm E=\left(J+\frac{1}{2}\right)-\left(K+\frac{1}{2}\right), \quad K=0,1,2 \ldots
$$

These are, as expected, the exact eigenvalues of $J_{x}:-J,-J+1, \ldots, J-1, J$.

Analytical expressions are also available for the eigenvectors. Thus the extreme eigenvalues are (unnormalized) $\sum_{M} a_{M} \mid J M>$ with

$$
a_{M}=( \pm)^{M} \exp \left[-M^{2} /(2 J+1)\right]
$$

The next eigenstates with $K= \pm(J-1)$ are described by

$$
a_{M}=( \pm)^{M} M \exp \left[-M^{2} /(2 J+1)\right]
$$

with further eigenstates involving successive Hermite polynomials multiplying the exponential factor. Considering that we are diagonalizing $J_{x}$ in a $\mid J M>$ basis, the eigenvectors are, of course, given exactly by $d_{M K}^{J}(\pi / 2)$ which are known standard rotational functions [116]. The results in (A.7) and (A.8) represent, therefore, approximations to these functions for large $\mathrm{J}$.

As a first step in the verification of the above results, we have, for instance, for the extreme value $K=J$, the exact result [116]

$$
d_{M J}^{J}(\pi / 2)=(-1)^{J-M} 2^{-J}[(2 J) ! /(J+M) !(J-M) !]^{1 / 2}
$$

which, upon use of the Stirling formula for factorials, gives

$$
d_{M J}^{J}(\pi / 2) \simeq(-1)^{J-M}\left[\pi\left(J+\frac{1}{2}\right)\right]^{-1 / 4} \exp \left[-M^{2} /(2 J+1)\right],
$$

coinciding precisely with the normalized form of (A.7). The approximations in (A.7) and (A.8) work well already at small $J$. For $J=\frac{1}{2}$, of course, we have only 
Table A.3. Rotation functions $d_{M K}^{J}(\pi / 2)$ for $J=2$ as given by (A.7) that follow from the spheroidal equation (A.5) that describes the classical limit of large $J$, contrasted with the exact values.

\begin{tabular}{lllllllllll}
\hline \hline & \multicolumn{4}{c}{ From Eqs. (A.7) and (A.8) } & \multicolumn{5}{c}{ Exact } \\
$\mathrm{K} \backslash \mathrm{M}$ & 2 & 1 & 0 & -1 & -2 & 2 & 1 & 0 & -1 & -2 \\
\hline 2 & 0.2712 & -0.5227 & 0.5536 & -0.5227 & 0.2712 & $\frac{1}{4}$ & $-\frac{1}{2}$ & $\left(\frac{3}{8}\right)^{1 / 2}$ & $-\frac{1}{2}$ & $\frac{1}{4}$ \\
1 & 0.4942 & -0.4762 & 0.2408 & 0.4762 & -0.4942 & $\frac{1}{2}$ & $-\frac{1}{2}$ & 0 & $\frac{1}{2}$ & $-\frac{1}{2}$ \\
0 & 0.6037 & 0 & -0.5207 & 0 & 0.6037 & $\left(\frac{1}{8}\right)^{1 / 2}$ & 0 & $-\frac{1}{2}$ & 0 & $\left(\frac{3}{8}\right)^{1 / 2}$ \\
-1 & 0.4942 & 0.4762 & 0.2408 & -0.4762 & -0.4942 & $\frac{1}{2}$ & $-\frac{1}{2}$ & 0 & $-\frac{1}{2}$ & $-\frac{1}{2}$ \\
-2 & 0.2712 & 0.5227 & 0.5536 & 0.5227 & 0.2712 & $\frac{1}{2}$ & $\frac{1}{2}$ & $\left(\frac{3}{8}\right)^{1 / 2}$ & $\frac{1}{2}$ & $\frac{1}{4}$ \\
\hline \hline
\end{tabular}

the two extreme eigenvectors so that we have only (A.7), and normalization alone is sufficient to fix the exact entries $\pm 1 / \sqrt{2}$ in the $d$ function. $J=2$ affords a less trivial illustration and Table A.3 compares the results from (A.7) and (A.8) with the exact values of $d$. The analytical spheroidal treatment of the operator $J_{x}$ can be seen, therefore, as a way of getting the classical limit $(J>>1)$ of these angular functions.

It is also instructive to examine the localization in real space of these eigenvectors, that is, $f(\varphi)=\sum_{M} a_{M} \exp (-i M \varphi)$. Thus the extreme eigenvectors from (A.7) give the distributions

$$
\sum_{M}( \pm)^{M} \exp \left[-i M \varphi-M^{2} /(2 J+1)\right] \simeq \exp \left[-(2 J+1) / 4\left(\begin{array}{c}
\varphi^{2} \\
(\varphi-\pi)^{2}
\end{array}\right)\right]
$$

which are localized Gaussians around the $\varphi=0$ and $\pi$ directions. Fig. 21 provides a comparison of these results (the normalization factors in (A.11) are $\left.[(2 J+1) / 2 \pi]^{1 / 4}\right)$ with the exact $f(\varphi)$, which are $\pi^{-1 / 2} \cos (\varphi / 2), \pi^{-1 / 2} \sin (\varphi / 2)$, and $(2 \pi)^{-1 / 2}\left[\frac{1}{2} \cos 2 \varphi-\cos \varphi+\left(\frac{3}{8}\right)^{1 / 2}\right]$. 

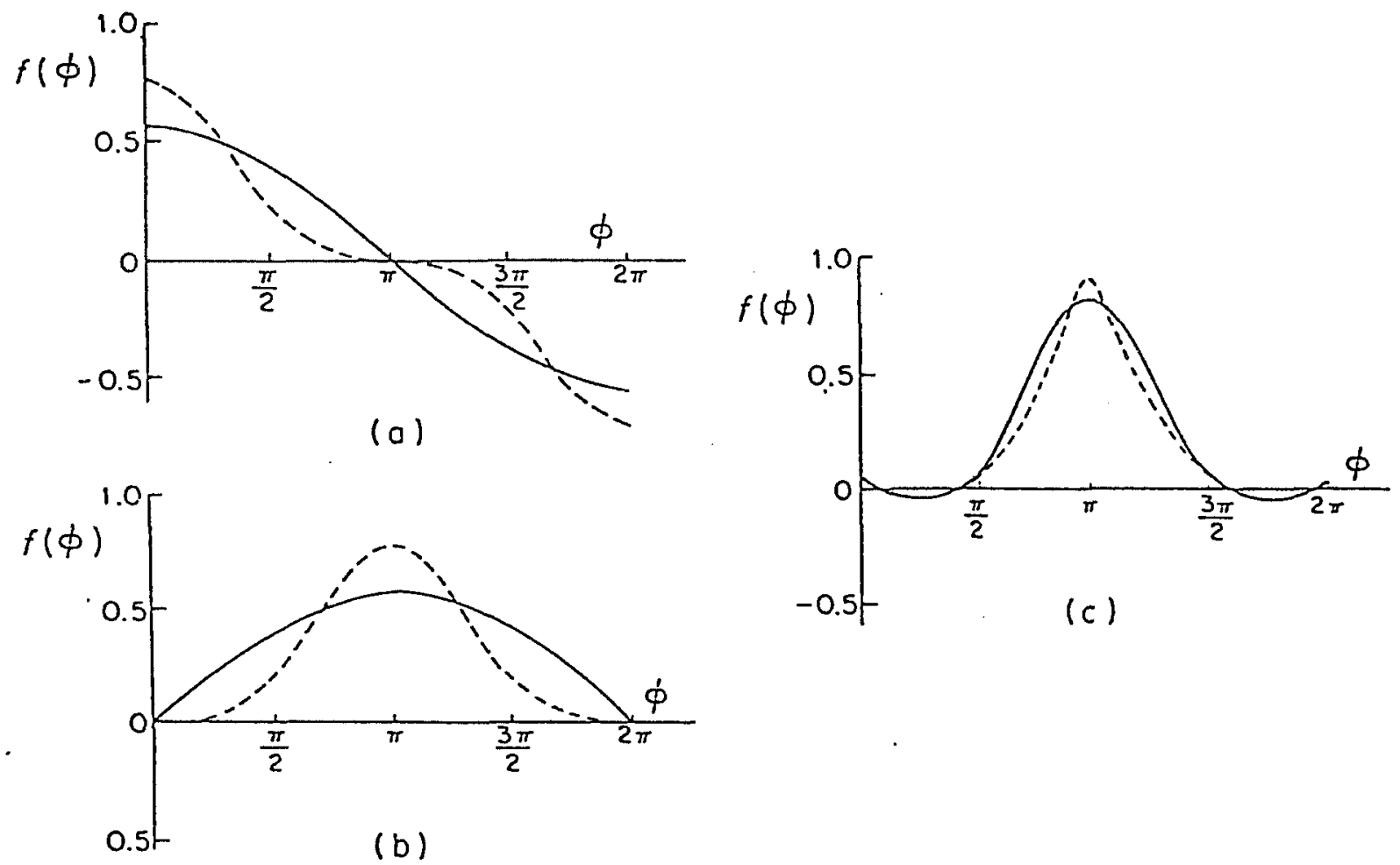

Figure 21. Distribution in coordinate space of eigenvectors $f(\varphi)$ of $J_{x}$ as given by Eq. (A.9) (- -), compared with the exact values (-). (a) $J=\frac{1}{2}$, eigenvalue $K=+\frac{1}{2}$, exact $f(\varphi)=\pi^{-1 / 2} \cos (\varphi / 2)$. (b) $J=\frac{1}{2}$, eigenvalue $K=-\frac{1}{2}$, exact $f(\varphi)=\pi^{-1 / 2} \sin (\varphi / 2)$. (c) $J=2, K=+2$, exact $f(\varphi)=(2 \pi)^{-1 / 2}\left[\frac{1}{2} \cos 2 \varphi-\right.$ $\left.\cos \varphi+\left(\frac{3}{8}\right)^{1 / 2}\right]$ 
A.3.2 Diagonalization of $J_{x}^{2} \quad$ Finding the eigenstates of the operator $J_{x}^{2}$ in the $\mid J M>$ basis is clearly immediately and intimately related to the above discussion of $J_{x}$. We should expect to find the same eigenvectors, and eigenvalues that are simply squares of (A.6). Indeed this is so. $J_{x}^{2}$ has the advantage of a more ready comparison with (A.1) and (A.2) of Sec. A.2 in that the matrix elements do not have square roots as in (A.4). In fact, the off-diagonal and diagonal elements are [116]

$$
\begin{aligned}
<J M+2\left|J_{x}^{2}\right| J M> & =[(J+M+2)(J-M-1)(J+M+1)(J-M)]^{1 / 2} / 4 \\
& \simeq\left[J(J+1)-(M+1)^{2}\right] / 4, \\
<J M-2\left|J_{x}^{2}\right| J M> & =[(J-M+2)(J+M-1)(J+M)(J-M+1)]^{1 / 2} / 4 \\
& \simeq\left[J(J+1)-(M-1)^{2}\right] / 4, \\
<J M\left|J_{x}^{2}\right| J M> & =\left[J(J+1)-M^{2}\right] / 2 .
\end{aligned}
$$

Therefore the eigenvalue problem of $J_{x}^{2} / J(J+1)$ is precisely of the form (A.1),

$$
W_{M-1} a_{M-2}+V_{M} a_{M}+W_{M+1} a_{M+2}=E a_{M}
$$

with $W_{M}$ and $V_{M}$ as in (A.2), except that now $u \equiv M /\left(J+\frac{1}{2}\right)$. The values of the coefficients are

$$
\omega=\frac{1}{4}, \quad v_{0}=v_{1}=\frac{1}{2} .
$$

Through the spheroidal equation that follows upon inserting (A.16) in (A.3), we find eigenvalues $K^{2} / J(J+1), K=0,1,2, \ldots$, and the same eigenvectors as in (A.7) and (A.8). For future use below, we note that for the operator $J_{y}^{2}$ the matrix elements are just as in (A.12), (A.13) and (A.14) except for a change of sign in the off-diagonal entries. This means $\omega$ alone in (A.16) has a change in sign. 


\section{A.3.3 The Asymmetric Rotor The eigenvalue problem of}

$$
H=\left(A J_{x}^{2}+B J_{y}^{2}+C J_{z}^{2}\right) / J(J+1)
$$

is clearly also of the form in (A.15) or the equivalent differential equation in (A.3). From the entries in (A.16) for $J_{x}^{2} / J(J+1)$ and equivalent ones for the other two operators, we have for (A.17)

$$
\omega=(A-B) / 4, \quad v_{0}=(A+B) / 2, \quad v_{1}=-C+(A+B) / 2 .
$$

Inverting this set of equations, we have

$$
A=v_{0}+2 \omega, \quad B=v_{0}-2 \omega, \quad C=v_{0}-v_{1}
$$

From the previous discussion of the nature of the solutions of the general spheroidal equation, the three combinations of $\omega, v_{0}$, and $v_{1}$ in (A.19) are precisely the extreme eigenvalues and the eigenvalue of the separatrix. In the asymmetric rotor, the fact that these values coincide with the three coefficients in the Hamiltonian in (A.17) points immediately to an association with three types of localization, those in which the angular momentum points in the direction of one of the three axes. The intermediate value characterizes the separatrix and is the $z$ axis for all but one of the problems considered in Sec. A.2. The conjugation link between the two ends of the spectrum, which is described by $\omega \rightarrow-\omega$, interchanges $A$ and $B$ in (A.19) and is, therefore, an interchange of the $\mathrm{x}$ and $\mathrm{y}$ axes, the two with the largest and smallest moments of inertia.

The literature on the asymmetric rotor recasts the Hamiltonian in (A.17) in a form that more transparently exhibits the asymmetry

$$
H=\frac{1}{2}(A+C)+[(A-C) / 2 J(J+1)]\left(J_{x}^{2}+\kappa J_{y}^{2}-J_{z}^{2}\right),
$$


with an "asymmetry parameter" defined by [104-105]

$$
\kappa=(2 B-A-C) /(A-C)
$$

This parameter is sometimes denoted by $\chi$ in the literature. Apart from a constant term $A+C$, the Hamiltonian is therefore characterized by an "asphericity" parameter $(A-C) /(A+C)$ and the asymmetry. Instead of three coefficients, a single one $\kappa$, suffices to discuss the significant part of an asymmetric rotor Hamiltonian $J_{x}^{2}+\kappa J_{y}^{2}-J_{z}^{2}$. From (A.19) and (A.21), $\kappa$ can be expressed in terms of the triplet of interest to us

$$
\kappa=\left(v_{1}-6 \omega\right) /\left(v_{1}+2 \omega\right) .
$$

The single parameter that is equivalent to $\kappa$ is, therefore, $\omega / v_{1}$.

It is also customary in the literature on the asymmetric rotor to standardize the labeling of the axes so that $A \geq B \geq C$ in which case $\kappa$ takes values in the interval $(-1,1)$. For the applications we have in mind, however, where the quantization or field direction is taken to be the $\mathrm{z}$ axis and this marks the separatrix, it is the coefficient $C$ that takes the intermediate value and $\kappa$ for such applications is less than -1 [117]. In Fig. 22 we present eigenvalues for a range $-1 \leq \kappa \leq 1$. The eigenvalues lie between -1 and 1 with a separatrix at $\kappa$. The eigenvalues come in degenerate pairs in the interval $(-1, \kappa)$. For $\kappa>1$, the eigenvalues lie between -1 and $\kappa$ with the separatrix at the fixed value 1 , with these three numbers corresponding to localization along the $z, y$, and $\mathrm{x}$ axis, respectively. For $\kappa<-1$, the range of eigenvalues is $\kappa$ to 1 and the separatrix frozen at -1 in between, the localizations being along $y, x$, and $z$ axis, respectively. This last case is the one of most interest, as discussed below in Sec. A.4, for the physical problems listed in Sec. A.2 and Table A.1. 


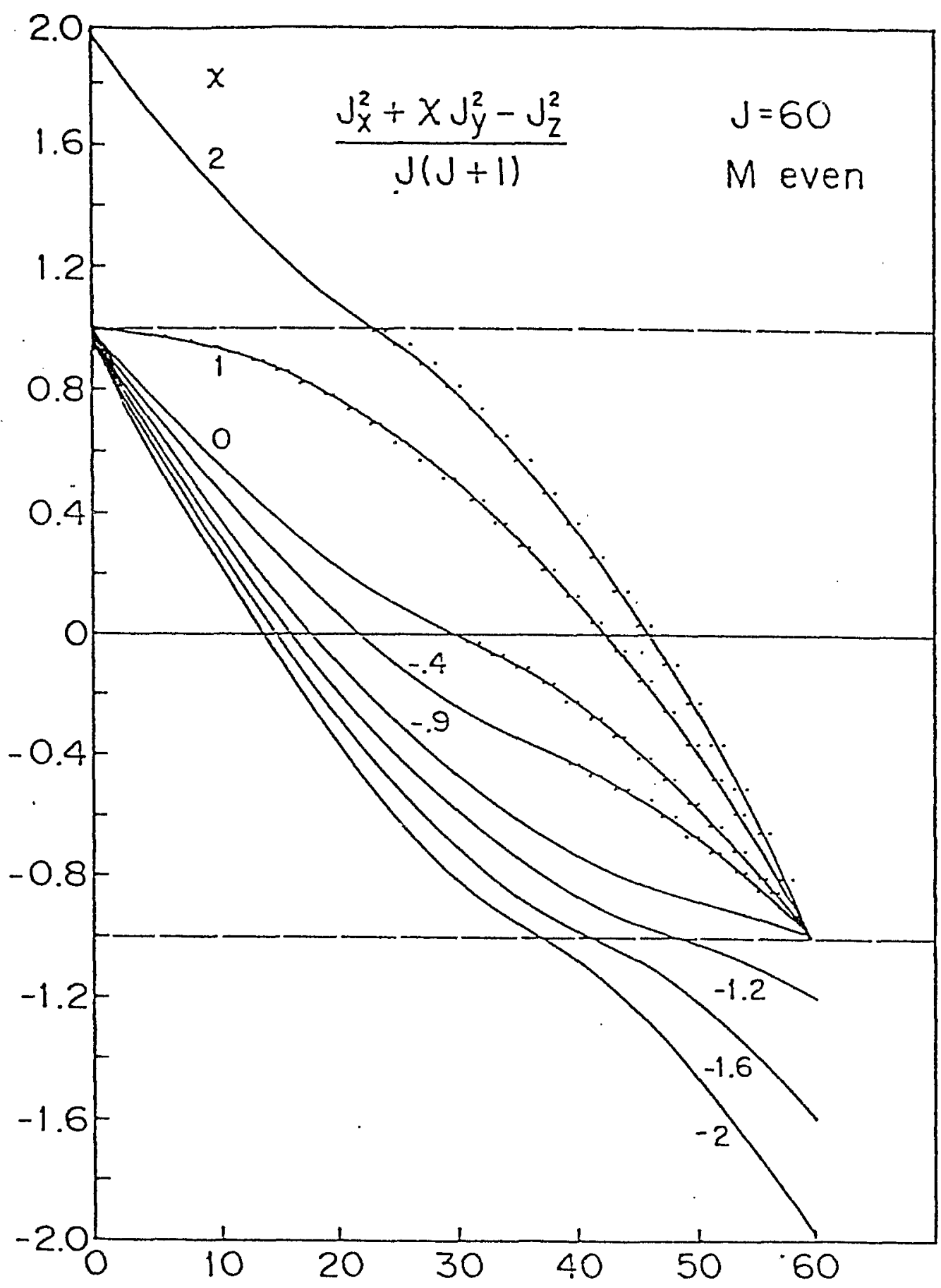

115

\section{Eigenstate}

Figure 22. Eigenvalues of the asymmetric rotor $\left(J_{x}^{2}+\kappa J_{y}^{2}-J_{z}^{2}\right) / J(J+1)$ for $\mathrm{J}=60$ and various values of the asymmetry parameter $\kappa$, shown as continuous curves drawn through the 61 eigenvalues labeled $0-60$ on the abcissa. In the range $|\kappa| \leq 1$, the separatrix lies at the value $\kappa$, otherwise at $\kappa /|\kappa|$. For $\kappa>-1$, the eigenvalues below the separatrix come as pairs of degenerate values indicated by the dots. 
The expression of the asymmetric parameter $\kappa$ in terms of $\omega / v_{1}$ in (A.22), when combined with the conjugation operator $\omega \rightarrow-\omega$, points to pairs of conjugate rotors that do not seem to have been recognized so far in the literature on asymmetric rotors. Under this reversal of sign, $\kappa$ goes over to $\tilde{\kappa}$ which is related to it by

$$
\kappa \rightarrow \tilde{\kappa}=(3-\kappa) /(1+\kappa)
$$

Eigenvalues for asymmetries $\kappa$ and $\tilde{\kappa}$ are simply related through an interchange between the two sets on either side of the separatrix. The interval $\kappa=(-\infty,-1)$ that is of most interest to us in this work maps into itself under the transformation in (A.23). It is also useful to consider a related angle parameter, usually defined [118] for the interval $\kappa=(-1,1)$ through $\kappa=\cos 2 \gamma$. In terms of this parameter $\gamma$, the operation in (A.23) amounts to taking the reciprocal of $\cos ^{2} \gamma$, that is, $2 \cos ^{2} \gamma-1 \rightarrow\left(2 / \cos ^{2} \gamma-1\right)$. The introduction of this new parameter for the interval $\kappa=(-\infty,-1)$ can be achieved through the definition

$$
\kappa=-\cosh 2 \gamma,
$$

in terms of which, once again, the transformation in (A.23) takes the form

$$
-1-2 \sinh ^{2} \gamma \rightarrow-1-\left(2 / \sinh ^{2} \gamma\right)
$$

\section{A.4 Mapping Problems Onto The Asymmetric Rotor}

The preceding section on angular momentum has been cast in a language that makes the correspondence with the problems in Sec. A.1 immediate. We start with the first examples considered in the two sections, namely, the linear Stark effect and the diagonalization of $J_{x}$. Unlike all the other cases considered involving quadratic operators, these two problems involve only the 
off-diagonal matrix elements $W$, with the diagonal $V$ being zero. Also, they have a square-root structure for $W$ as in (A.4) in place of the form in (A.2). Both these problems have an identical structure, namely, $W \propto\left(1-u^{2}\right)^{1 / 2}$, with $u=\left(l+\frac{1}{2}\right) / n$ or $M /\left(J+\frac{1}{2}\right)$. The results are, therefore, not surprisingly identical. The equally spaced eigenvalues of the linear Stark effect are, therefore, seen in a new light, as in immediate correspondence with the familiar fact of equal spacing (the set of integers) for the projection of an angular momentum in quantum mechanics. The role of $J$ and $M$ in the rotor is played by $n$ and $l$, respectively, in the Stark effect. A degenerate hydrogenic manifold, $l=0,1,2, \ldots, n-1$, is in $1: 1$ correspondence with the space of angular momentum $J: M=-J,-J+1, \ldots, J-1, J$. With $(2 J+1)=n$ and $J+M=l$, the mapping is complete. We note that the association of an angular momentum $J=(n-1) / 2$ with the $n$th manifold is reminiscent of the well-known [119] $O_{4}$ description of the hydrogen atom, but has, in fact, nothing to do with it. In the $O_{4}$ picture, each manifold is associated with twin angular momenta $j_{1}$ and $j_{2}$, both of the same magnitude $(n-1) / 2$. All $n^{2}$ degenerate states are embraced in this representation [with $\left.\left(2 j_{1}+1\right)\left(2 j_{2}+1\right)=n^{2}\right]$ and the angular momentum $l$ is viewed as the sum $j_{1}+j_{2}$. Such a sum also marks the passage from parabolic to spherical basis, the former being associated with $j_{1}^{2}, j_{2}^{2}, j_{1 z}, j_{2 z}$. Our association is different. We are considering only spherical states of the $n$ manifold with a fixed azimuthal quantum number $m$. For $m=0$, this is the $n$-fold degenerate set of $l$ values listed above and this set is regarded as the $(2 J+1)$ degenerate states of a single angular momentum $(n-1) / 2$.

The mapping of all the other problems considered in Sec. A.2, and which are listed in Table A.1, into the asymmetric rotor Hamiltonian in (A.17) proceeds through (A.1) and (A.15). These difference equations are in the same 
common form in (A.2). The variable $u$ is $\left(l+\frac{1}{2}\right) / n$ for the problems in Table A.1 and $M /\left(J+\frac{1}{2}\right)$ for the asymmetric rotor. Once this variable is treated as a continuous variable, the two are clearly identical. The two intervals are $(0,1)$ and $(-1,1)$, respectively, and this difference allows alternative choices for the mapping of Table A.1 into an asymmetric rotor. One choice is $J \rightarrow(n-1) / 2$, $M \rightarrow l+\frac{1}{2}$, another $J \rightarrow l+\frac{1}{2}, M \rightarrow l+\frac{1}{2}$. Both have been used in the results presented below. The asymmetry parameter $\kappa$ is uniquely fixed for each problem through (A.22) and is shown in the last column of Table A.1. From Table A.1 and (A.18), the diamagnetic problem maps onto the asymmetric rotor $\left(5 J_{x}^{2}+J_{z}^{2}\right) / 4 J(J+1)$ with asymmetry parameter $\kappa=-9$. Fig. 23 is a plot of the eigenvalues for the asymmetric rotor with $\mathrm{J}=50.5$ and 25 . On the same plot are marked the diamagnetic eigenvalues for states with zero azimuthal quantum number in the $n=51$ manifold. Note the close correspondence of the results, the $J=n-\frac{1}{2}$ choice doing slightly better in the region above the separatrix and the $J=(n-1) / 2$ below the separatrix. As an application of the passage through (A.23) to an equivalent rotor, note that $\tilde{\kappa}=-\frac{3}{2}$, which describes $\left(5 J_{x}^{2}+J_{z}^{2}\right) / 4 J(J+1)$, also describes the same spectrum. The entries in Table A.1 for the extrema merely interchange their positions.

The quadrupole interaction between two nucleons has $\kappa=-3$, a value shared with all problems which have $v_{1}=0,($ see (A.22)). Fig. 24 compares eigenvalues for $n=39^{1} S$ states with those of asymmetric rotors with $J=38.5$ and 19. Once again it is clear that the asymmetric rotor with $\kappa=-3$ does indeed describe this system. [Note also from (A.23) that $\tilde{\kappa}$ is also -3 for this system.] Also shown in the insets are the eigenvectors for the rotor's extreme states and the separatrix. Note that the latter is distributed over all $M$ values with a slight excess of $|M| \simeq J$, whereas the extreme ones show a Gaussian 


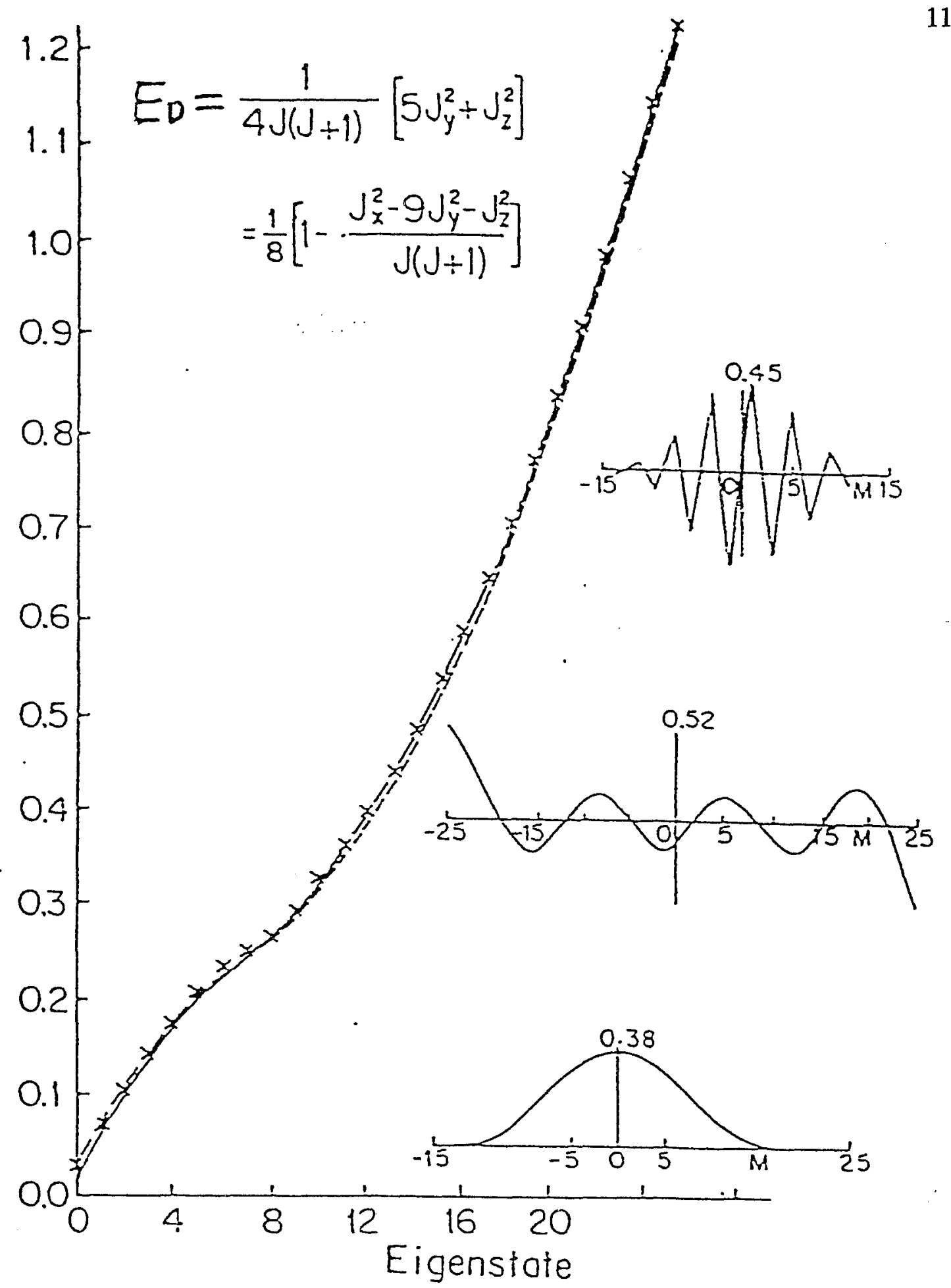

Figure 23. Eigenvalues $E_{D}$ of the asymmetric rotor with $\kappa=-9$, and $J=50.5$ (-) and $J=25(--)$, along with $M$ distributions of the extreme states (highest and lowest eigenvalues) and the separatrix in between. Note the close similarity of the two plots of eigenvalues. Eigenvectors are also similar in structure and only the $J=25, M$ odd, are shown. Crosses represent the eigenvalues of the diamagnetic interaction for $\mathrm{n}=51$ (see Table A.2). 
falloff in $M^{2} /(2 J+1)$. In addition, between the highest and lowest eigenvectors there is an alternating sign difference in the coefficients. All these features mirror exactly the similar behavior in $l$ of the diamagnetic eigenvectors (Fig. 4 of Ref. 96). There is an immediate geometric understanding of these behaviors in the rotor problem. The separatrix, with localization along the $\mathrm{z}$ axis, is the only one which has a large presence of the high $|M|$ values. The extreme states are in the xy plane and are built out of superpositions of states with low to intermediate $|M|,|M|<J^{1 / 2}$.

Plots of the eigenvalues of $n^{2} a_{0} / r_{12}$ for two electrons in the nth shell in ${ }^{1} S$ states are shown in Fig. 25. As can be seen from Table A.1, nearly all the models for this interaction give asymmetry parameters which are either very large and negative or equal to -1 , and these are equivalent according to (A.24). As a result the distribution of eigenvalues in the plot closely approximates that of a symmetric rotor, $K^{2} / J(J+1),|K|=0,1,2, \ldots$.

Finally, the Edmonds-Pullen model differs from the others in Table A.1 in having its extreme eigenvalues localized along $\mathrm{x}$ and $\mathrm{z}$ (not $\mathrm{y}$ ) axes of the rotor. Localization along the rotor's y axis now marks the separatrix. Note also from Ref. 100 that $u$ for this model is defined as $1-2\left(n_{x} / n\right)$, where $n_{x}$ is the quantum number of the $\mathrm{x}$ oscillator, and $n=n_{x}+n_{y}$. The spheroidal equation in (A.3) has a factor $n^{2} / 16$ in place of $n^{2} / 4$.

\section{A.5 Summary and Geometrical Interpretation}

The study of eigenvalues of angular momentum operators such as $J_{x}$ or $J_{x}^{2}$ at a fixed, high angular momentum $J$ provides a simple illustration of the diagonalization of tridiagonal matrices in a large degenerate manifold. Diverse one- and two-particle problems in atomic physics and elsewhere, which are of 


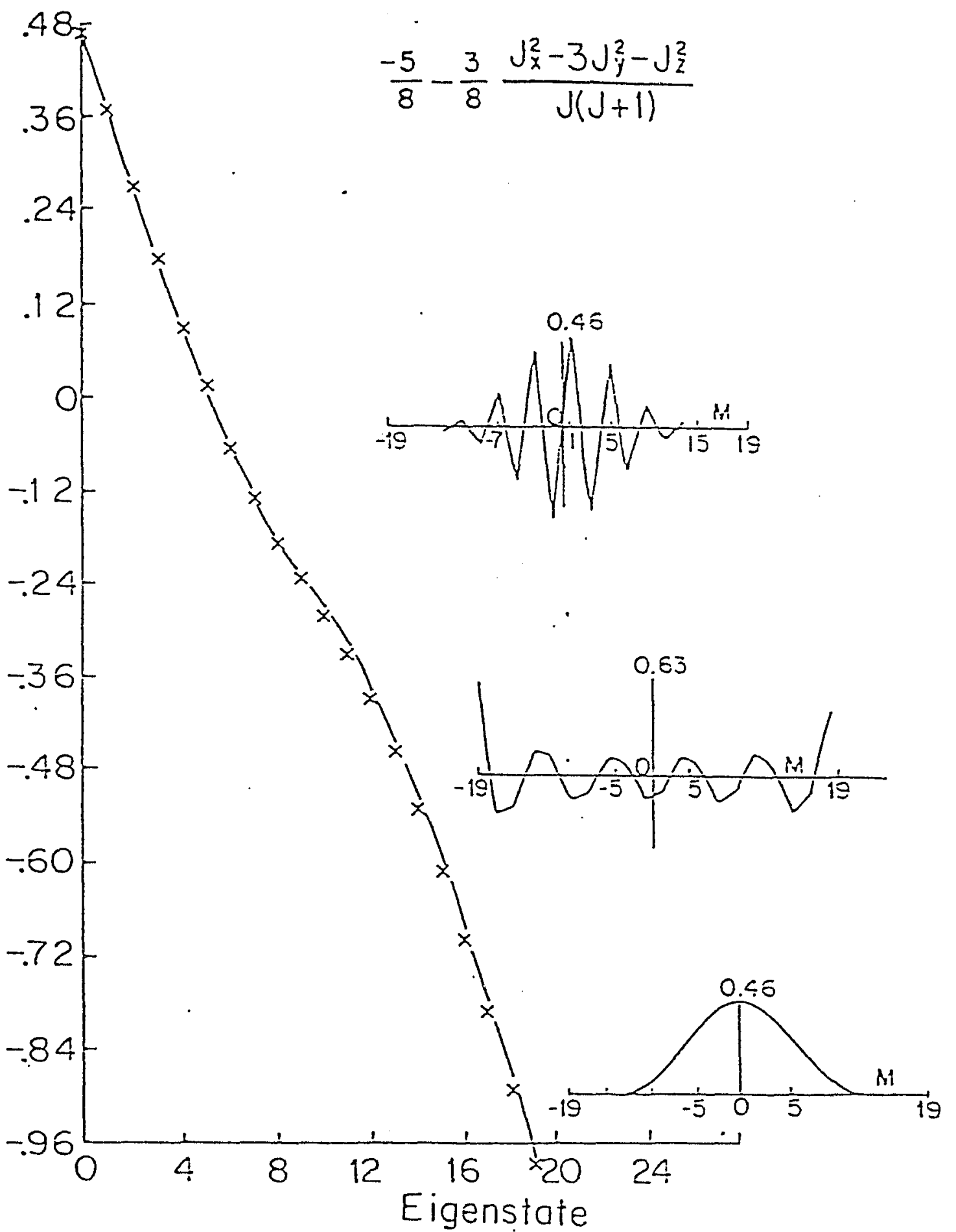

Figure 24. Similar to Fig. 23 but for the quadrupole-quadrupole interaction $E_{Q}$ between nucleons. Eigenvalues of the asymmetric rotor with $\kappa=-3$ and $J=38.5$ and 19 (which are essentially indistinguishable on this scale) are shown by the solid line and compared with the nuclear problem $n=39$, denoted by crosses. Compare also the distribution of extreme eigenvectors and separatrix with the corresponding ones for the nuclear problem shown in Fig. 20. 


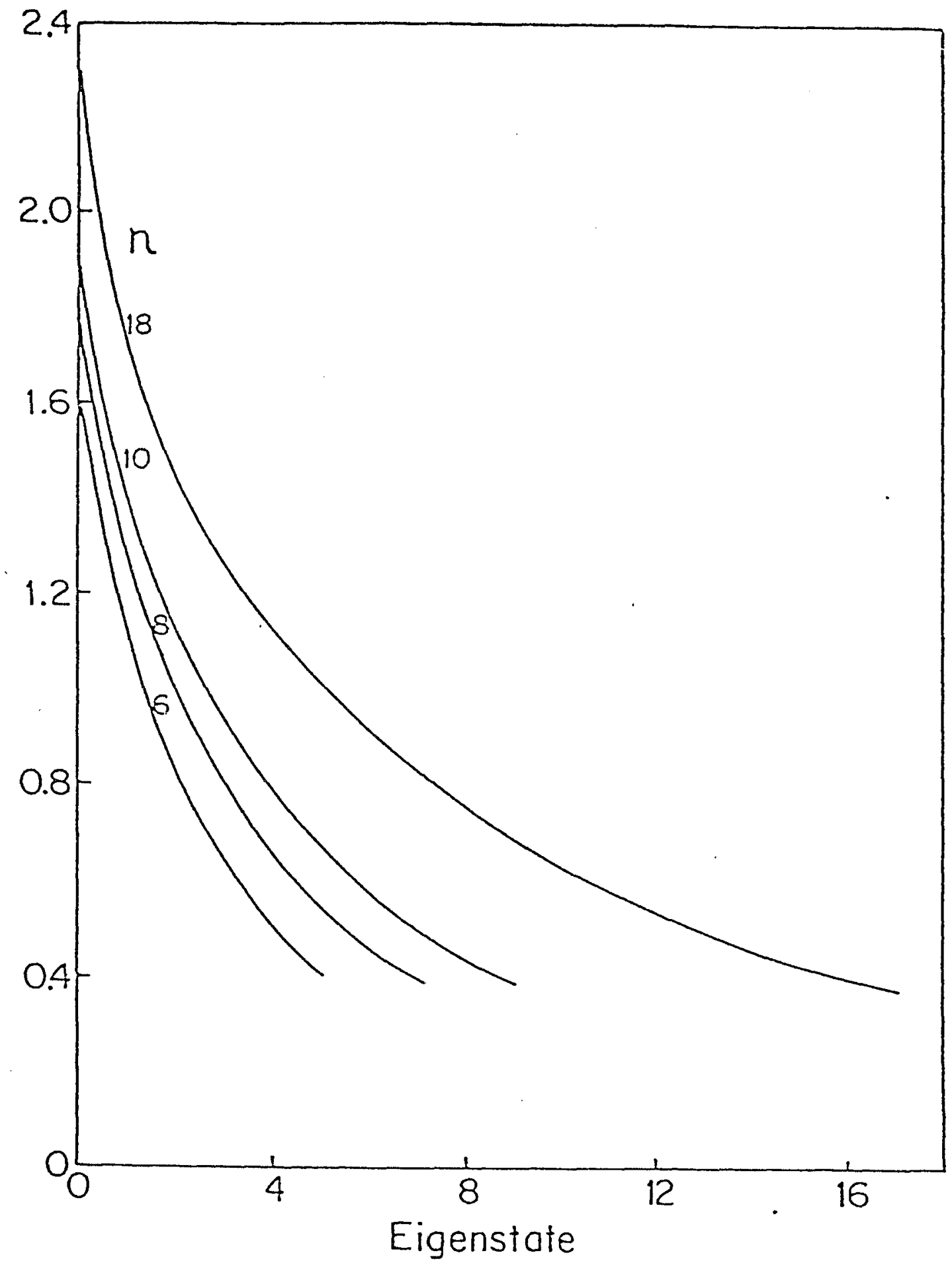

Figure 25. Distribution of the eigenvalues $E_{e}$ of the electron-electron interaction $n^{2} a_{0} / r_{12}$ between two hydrogenic electrons, both in the nth shell and coupled to ${ }^{1} S^{e}$ symmetry. The results are drawn from numerical calculations presented in Refs. 35, 71, and 101. 
current, active interest, involve the mixing of states of manifolds (with principal quantum number $n$ ) which have a large degeneracy. This mixing for a number of problems listed in Table A.1 was shown to be of exactly the same form as in the description of the asymmetric rotor, $A J_{x}^{2}+B_{y}^{2}+C J_{z}^{2}$. Indeed, a complete mapping was provided in Sec. 4 for each physical problem into the asymmetric rotor and the parameters $A, B$, and $C$ identified. Equivalently, in a standard form of the rotor Hamiltonian $\left(J_{x}^{2}+\kappa J_{y}^{2}-J_{z}^{2}\right) / J(J+1)$, the key parameter $\kappa$, called the asymmetry parameter, was identified. In the problems considered, $A \geq C \geq B$, and $\kappa<-1$. The role of $n$ and $l$ (angular momentum of the particle) in the $n$ physical problems is played by $J$ and $M$ in the asymmetric rotor.

A common feature that has been previously identified for all the physical problems-that, apart from a few eigenstates in the middle, nearly all the eigenvalues group into two classes of localized states-is connected to the similar structure exhibited by the states of the asymmetric rotor. The eigenvalues span the range $(\kappa, 1)$ - recall that $\kappa$ is less than -1 and the separatrix lies at -1 . These have clear identification with localization along the $y, x$ and $z$ axes, respectively. The first two are the axes with minimum and maximum moments of inertia and the $\mathrm{z}$ axis is intermediate between them. That the first two dominate the spectrum, accounting for most the physical eigenstates, can be understood in terms of the result for the asymmetric rotor that is already familiar in classical mechanics, namely, that only motions with respect to the extreme moments of inertia are stable, motion with respect to the intermediate axis being unstable. This can be seen most easily in the classical limit [120] 
of large $J$, when the asymmetric rotor Hamiltonian can be viewed as a purely geometrical object,

$$
H_{C L}=\sin ^{2} \theta\left(\cos ^{2} \varphi+\kappa \sin ^{2} \varphi\right)-\cos ^{2} \theta
$$

Expanding around each axis, we have for $\mathrm{x}(\theta \simeq \pi / 2, \varphi \simeq 0)$,

$$
1-2 \tilde{\theta}-(1-\kappa) \tilde{\varphi}^{2}
$$

for $y(\theta \simeq \pi / 2, \varphi \simeq \pi / 2)$

$$
\kappa-(1+\kappa) \tilde{\theta}^{2}+(1-\kappa) \tilde{\varphi}^{2}
$$

and for $z(\theta \simeq 0)$,

$$
-1+(1+\kappa) \tilde{\theta}^{2}+(1-\kappa) \tilde{\theta}^{2} \cos ^{2} \varphi
$$

In each case $\tilde{\theta}$ and $\tilde{\varphi}$ denote small departures from the corresponding values that identify the axes. The limiting values $\kappa$ and 1 for the eigenvalues and -1 for the separatrix are, of course, the results when $\tilde{\theta}=0$ and $\tilde{\varphi}=0$. Recalling that $\kappa<-1$, departures from these central values are in the same direction for localization around $\mathrm{x}$ and $\mathrm{y}$ (the former is a maximum, the latter a minimum), while for the intermediate $\mathrm{z}$ axis, departures in $\tilde{\theta}$ do not have a well-defined sign but vary with $\varphi$.

The different $M$ projections of angular momentum $J$ span a $(2 J+1)$ dimensional space. When $J$ is large, the discrete projections pass over essentially into a continuous distribution, thereby giving a familiar classical limit. Our analysis shows that, very similarly, the $\mathrm{n}$-fold degenerate $l$ values in an atomic $n$ manifold behave like an angular momentum space. As a result, mixings of these $l$ due to interaction which couple $l$ tridiagonally can be described 
equivalently in terms of the most general quadratic structure in angular momentum operators, namely, the asymmetric rotor. Further, such a Hamiltonian, which is tridiagonal in $M$, is characterized by off-diagonal matrix elements that vanish at the limits $|M| \rightarrow J$. This is, of course, because the quadratic operators built out of angular momentum generators are bilinear in the step-up and step-down operators whose matrix elements vanish at the limits. Exactly similarly again, the radial operators describing various interactions in physical systems have the same feature of zero off-diagonal matrix elements when $l \rightarrow n$. This feature, which can be cast in terms of radial step operators, is an expression of a finite cutoff at the upper end $(l=n-1)$ to the manifold. [The step operators on radial atomic functions are usually expressed in terms of their action at fixed $l$, in changing $n$ by unit steps: $n=(l+1),(l+2), \ldots$, the action having a cutoff at the lower end.]

When $n$ is large, we have equally a classical limit for the atomic problems. Mirroring the behavior of asymmetric rotor eigenfunctions, localized states, particularly around the two axes of extreme moments of inertia, dominate in the spectrum. Compact analytical expressions for eigenvalues and eigenvectors in this limit are provided by our analysis wherein, in this continuous limit, the difference equation of tridiagonalization is transformed into a spheroidal differential equation. An important conjugation transformation between the two groups of localized states also finds a ready interpretation as the exchange of the axes with the extreme moments of inertia in the asymmetric rotor; in turn it suggests the existence of conjugate pairs of rotors which share similar eigenvalue patterns. 


\section{APPENDIX B}

\section{SOURCE CODE}

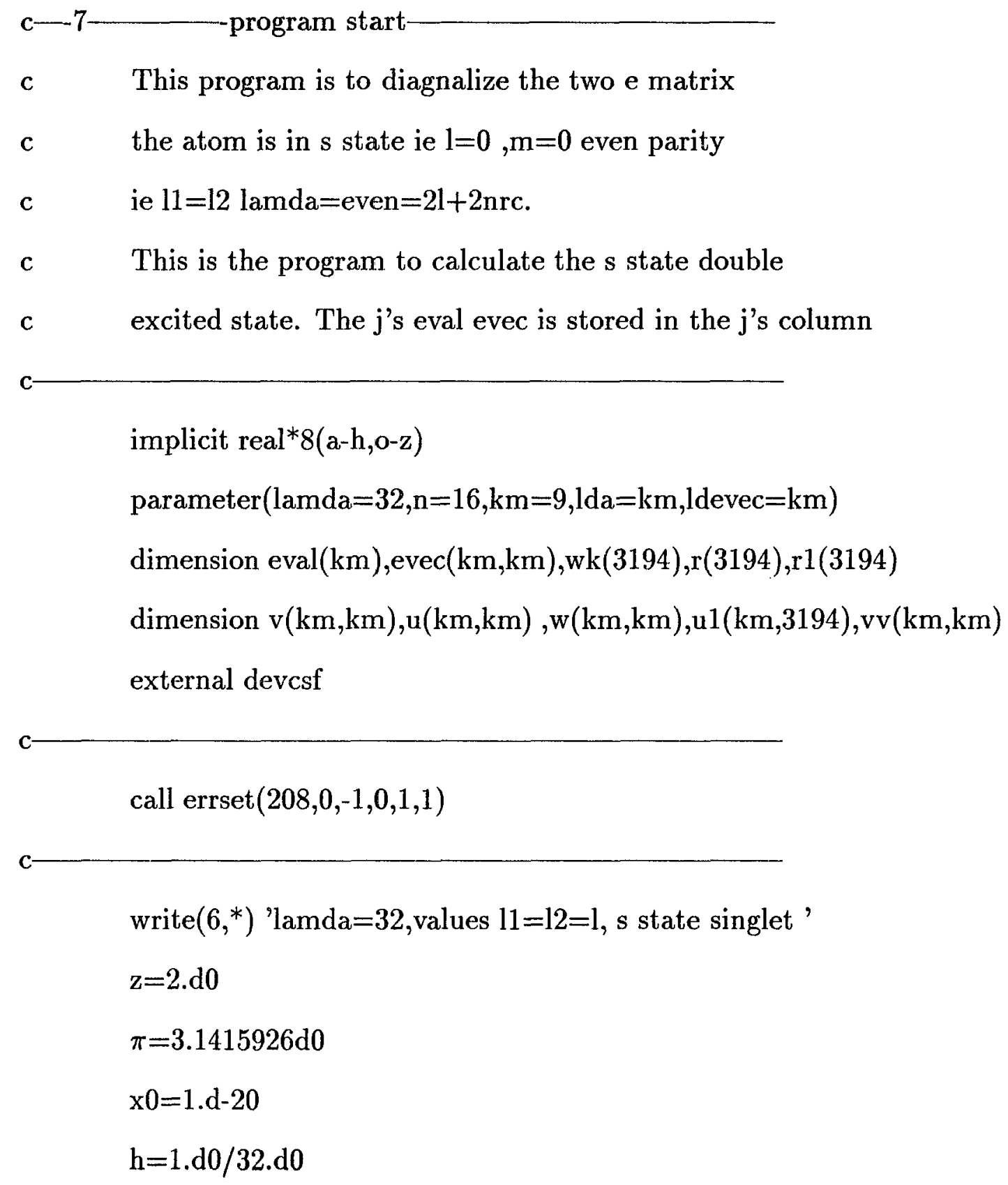




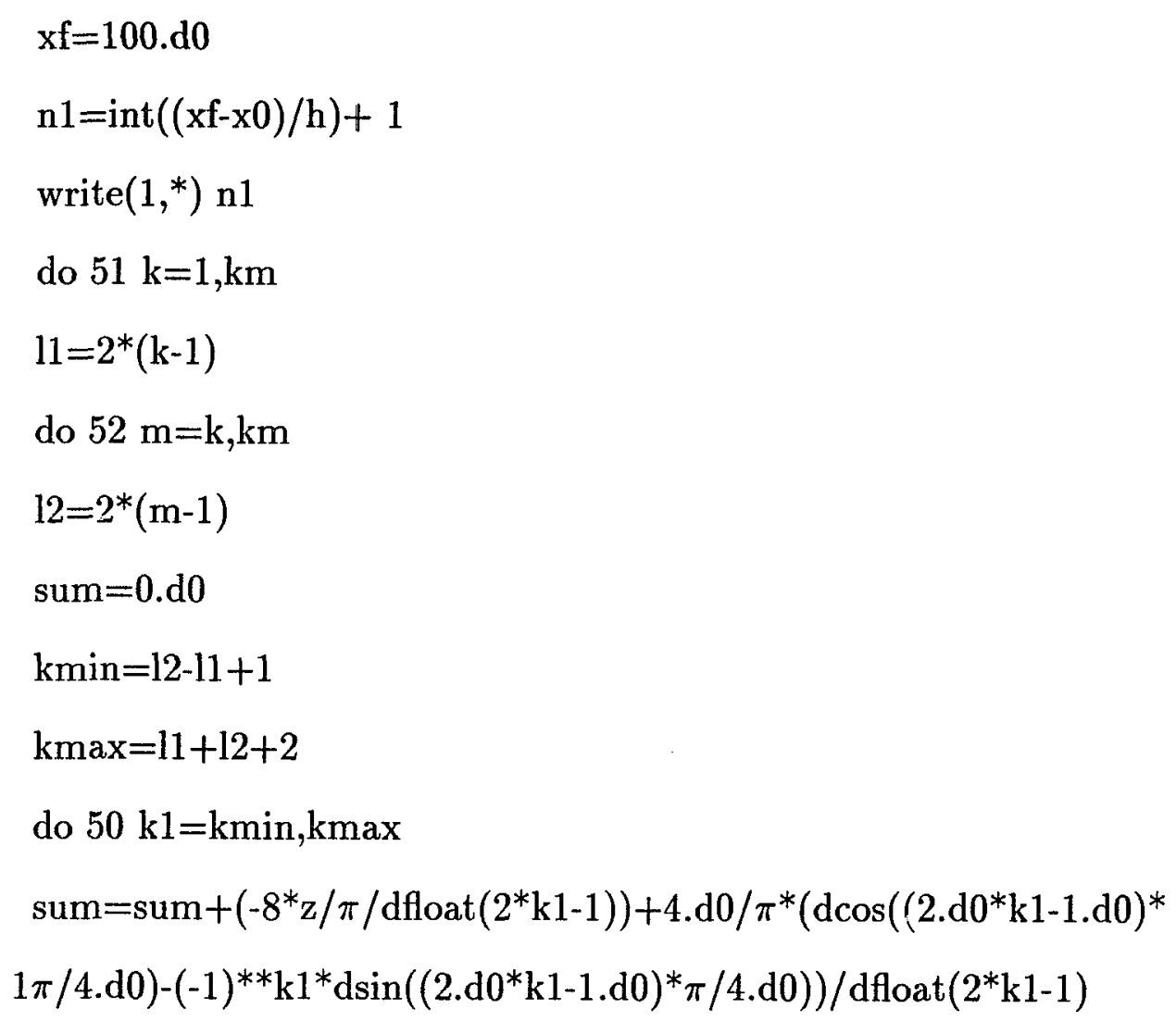

50 continue

$\mathrm{w}(\mathrm{k}, \mathrm{m})=\mathrm{sum}$

write $\left(6,{ }^{*}\right) \mathrm{w}(\mathrm{k}, \mathrm{m})$

52 continue

51 continue

do $111 \mathrm{ix}=1, \mathrm{n} 1$

$r(i x)=x 0+h^{*}$ dfloat $(i x-1)$

$\mathrm{r} 1(\mathrm{ix})=\operatorname{dsqrt}(\mathrm{r}(\mathrm{ix}))$

do $1 \mathrm{k}=1, \mathrm{~km}$

$11=2^{*}(\mathrm{k}-1)$

do $2 \mathrm{~m}=\mathrm{k}, \mathrm{km}$ 


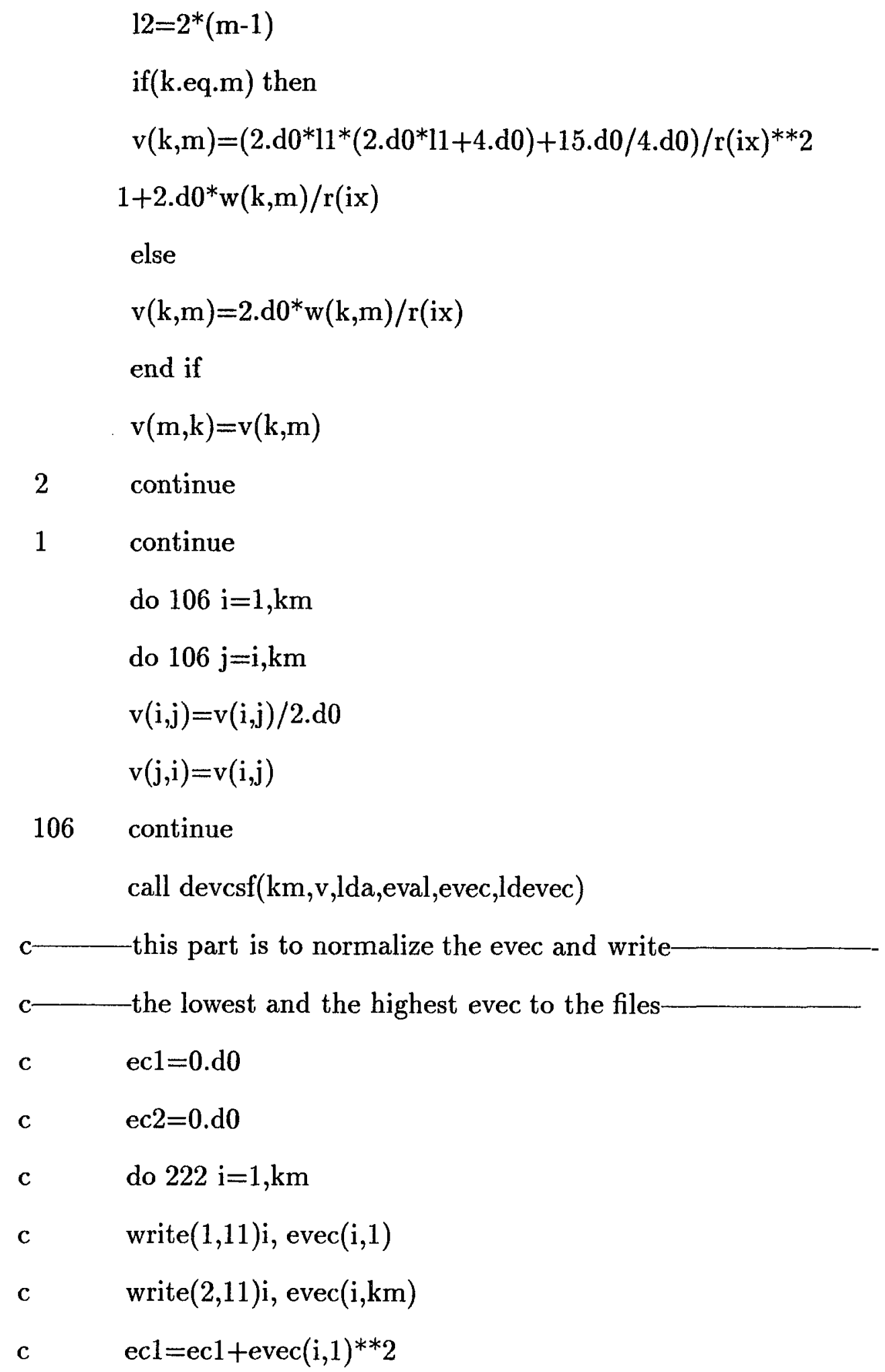




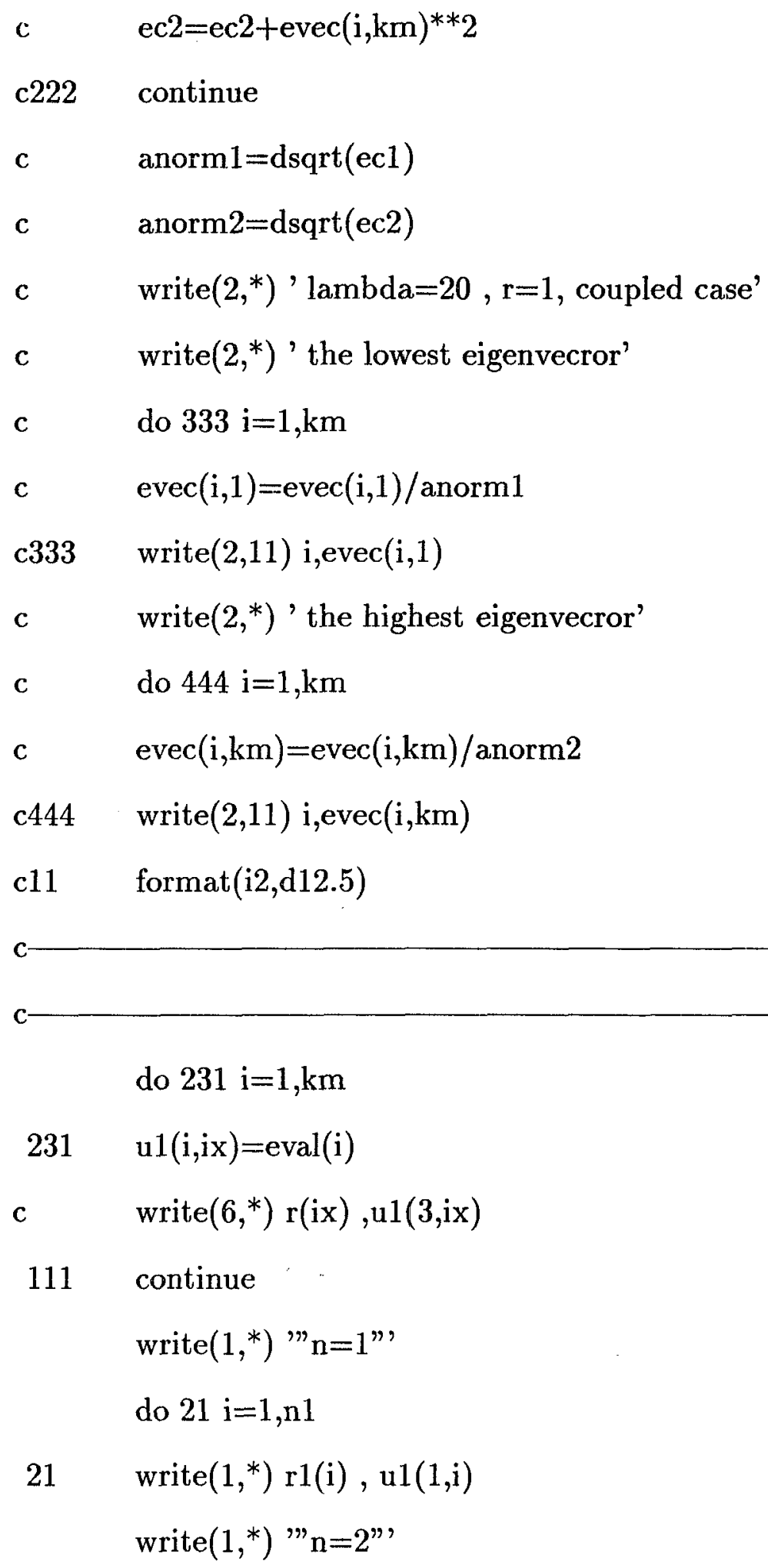




$$
\begin{aligned}
& \text { do } 22 \mathrm{i}=1, \mathrm{n} 1 \\
& 22 \quad \operatorname{write}\left(1,{ }^{*}\right) \mathrm{r} 1(\mathrm{i}), \mathrm{u} 1(2, \mathrm{i}) \\
& \operatorname{write}\left(1,{ }^{*}\right) ' n=3 " \\
& \text { do } 23 \mathrm{i}=1, \mathrm{n} 1 \\
& 23 \quad \text { write }\left(1,{ }^{*}\right) \mathbf{r} 1(\mathrm{i}), \mathbf{u} 1(3, \mathrm{i}) \\
& \text { write }(1, *) ' " n=4 " \\
& \text { do } 24 \mathrm{i}=1, \mathrm{n} 1 \\
& 24 \quad \operatorname{write}\left(1,{ }^{*}\right) \mathrm{r} 1(\mathrm{i}), \mathrm{ul}(4, \mathrm{i}) \\
& \text { write }(1, *) " n=5 " \\
& \text { do } 25 \mathrm{i}=1, \mathrm{n} 1 \\
& 25 \quad \text { write }\left(1,{ }^{*}\right) \operatorname{rl}(\mathrm{i}), \mathrm{u} 1(5, \mathrm{i}) \\
& \text { write }(1, *) " n=6 " \\
& \text { do } 26 \mathrm{i}=1, \mathrm{n} 1 \\
& 26 \quad \text { write }\left(1,{ }^{*}\right) \text { rl }(\mathrm{i}), \mathrm{u} 1(6, \mathrm{i}) \\
& \operatorname{write}\left(1,{ }^{*}\right) " n=7 " \\
& \text { do } 27 \mathrm{i}=1, \mathrm{n} 1 \\
& 27 \quad \operatorname{write}\left(1,{ }^{*}\right) \mathrm{r} 1(\mathrm{i}), \mathrm{u} 1(7, \mathrm{i}) \\
& \text { write }\left(1,{ }^{*}\right) " n=8 " \\
& \text { do } 28 \mathrm{i}=1, \mathrm{n} 1 \\
& 28 \quad \text { write }\left(1,{ }^{*}\right) \operatorname{rl}(\mathrm{i}), \mathrm{u} 1(8, \mathrm{i}) \\
& \text { write }\left(1,{ }^{*}\right) " n=9 " \\
& \text { do } 29 \mathrm{i}=1, \mathrm{n} 1 \\
& 29 \quad \operatorname{write}\left(1,{ }^{*}\right) \operatorname{rl}(\mathrm{i}), \mathrm{ul}(9, \mathrm{i}) \\
& \text { c } \quad \text { write }\left(2,{ }^{*}\right) " n=10 "
\end{aligned}
$$




\begin{tabular}{|c|c|}
\hline c & do $30 \mathrm{i}=1, \mathrm{n} 1$ \\
\hline c30 & $\operatorname{write}\left(2,{ }^{*}\right) \mathrm{r} 1(\mathrm{i}), \mathrm{u} 1(10, \mathrm{i})$ \\
\hline c & $\operatorname{write}(2, *) " ' n=11 "$ \\
\hline c & do $31 \mathrm{i}=1, \mathrm{n} 1$ \\
\hline c31 & $\operatorname{write}\left(2,{ }^{*}\right) \mathrm{r} 1(\mathrm{i}), \mathrm{u} 1(11, \mathrm{i})$ \\
\hline c & $\operatorname{write}(2, *) ' " n=12 "$ \\
\hline c & do $32 \mathrm{i}=1, \mathrm{n} 1$ \\
\hline c32 & $\operatorname{write}\left(2,{ }^{*}\right) \mathrm{r} 1(\mathrm{i}), \mathrm{u} 1(12, \mathrm{i})$ \\
\hline c & write $\left(2,{ }^{*}\right) " ' n=13 " '$ \\
\hline c & do $33 \mathrm{i}=1, \mathrm{n} 1$ \\
\hline c33 & $\operatorname{write}\left(2,{ }^{*}\right) \mathrm{r} 1(\mathrm{i}), \mathrm{u} 1(13, \mathrm{i})$ \\
\hline c & $\operatorname{write}\left(2,{ }^{*}\right) " n=14 " '$ \\
\hline c & do $34 \mathrm{i}=1, \mathrm{n} 1$ \\
\hline c34 & $\operatorname{write}\left(2,{ }^{*}\right) \mathrm{r} 1(\mathrm{i}), \mathrm{u} 1(14, \mathrm{i})$ \\
\hline c & write $\left(2,{ }^{*}\right) " n=15 "$ \\
\hline c & do $35 \mathrm{i}=1, \mathrm{n} 1$ \\
\hline c35 & $\operatorname{write}\left(2,{ }^{*}\right) \mathrm{r} 1(\mathrm{i}), \mathrm{u} 1(15, \mathrm{i})$ \\
\hline & end \\
\hline
\end{tabular}

c-

c-

function dfact(j)

if $(j$.eq. 0$)$ then

dfact $=1$.d 0

else 


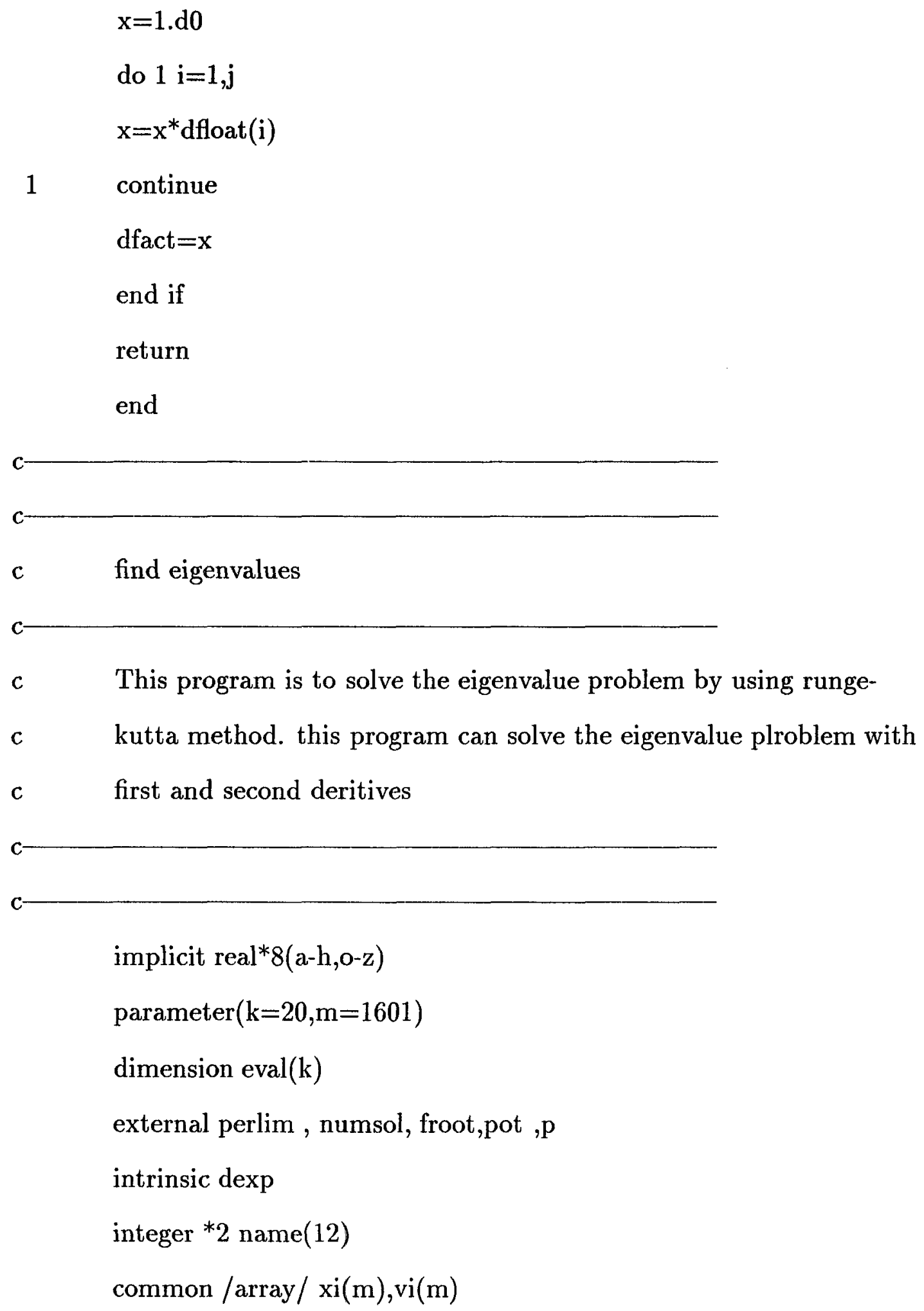




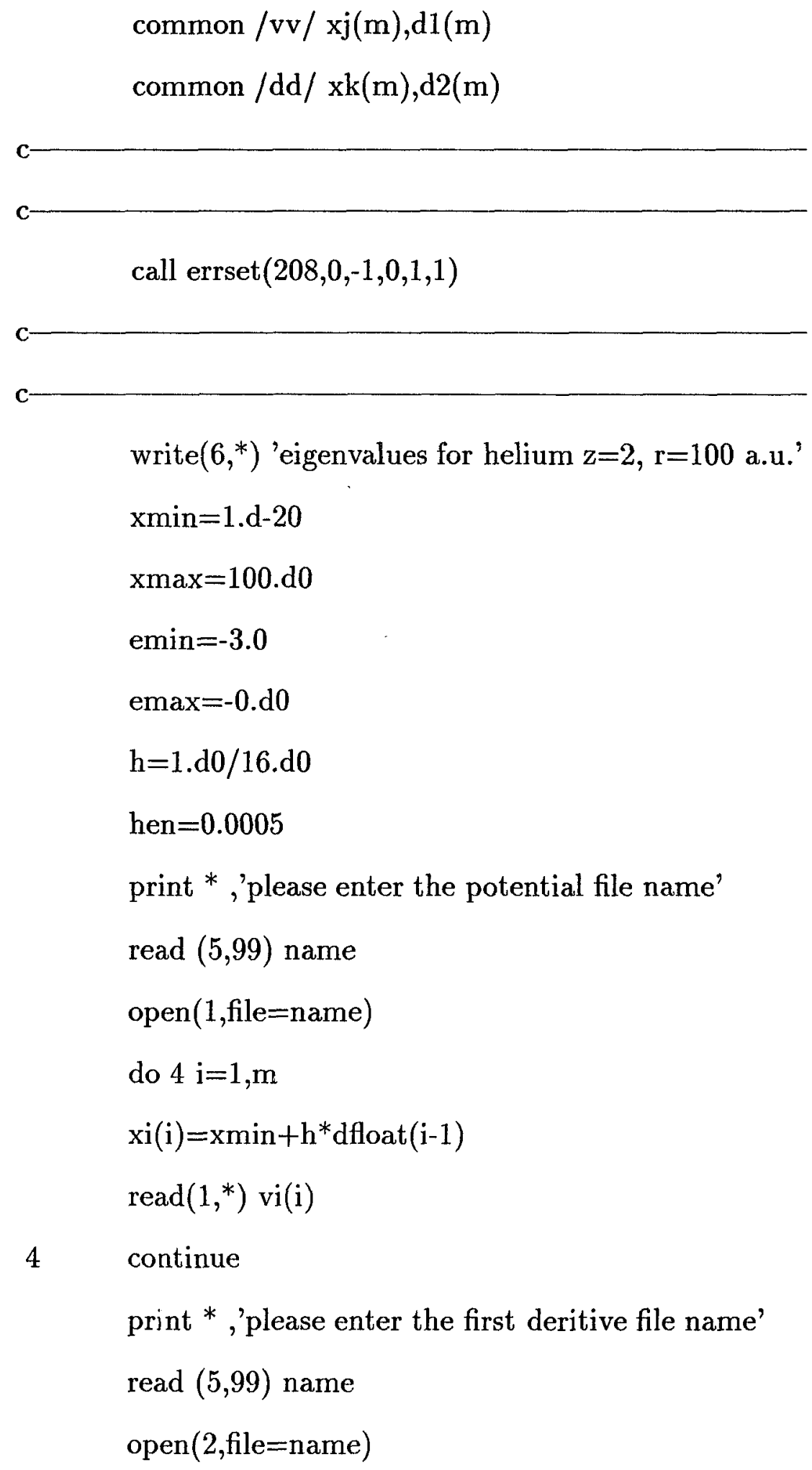


do $5 \mathrm{i}=1, \mathrm{~m}$

$\operatorname{read}\left(2,{ }^{*}\right) \operatorname{xj}(\mathrm{i}), \mathrm{d} 1(\mathrm{i})$

5 continue

print * ,'please enter the second deritive file name'

read $(5,99)$ name

open $(3$, file $=$ name $)$

do $6 \mathrm{i}=1, \mathrm{~m}$

$\operatorname{read}\left(3,{ }^{*}\right) \times \mathrm{k}(\mathrm{i}), \mathrm{d} 2(\mathrm{i})$

6 continue

99 format(12a2)

print *,'finishing reading data'

print *, 'please be patient to wait the result'

call perlim(xmin,xmax,h,nstep)

call numsol(emin,emax,hen,h,nstep,imax,eval)

do $2 \mathrm{i}=1$, imax

$\operatorname{eval}(\mathrm{i})=\operatorname{eval}(\mathrm{i}) / 2 . \mathrm{d} 0$

print ${ }^{*}$, ' the eigenvalue is ', eval(i)

2 continue

end

c—-function potential

c-

function $\operatorname{pot}(\mathrm{x})$

implicit real*8(a-h,o-z)

parameter $(m=1601)$ 


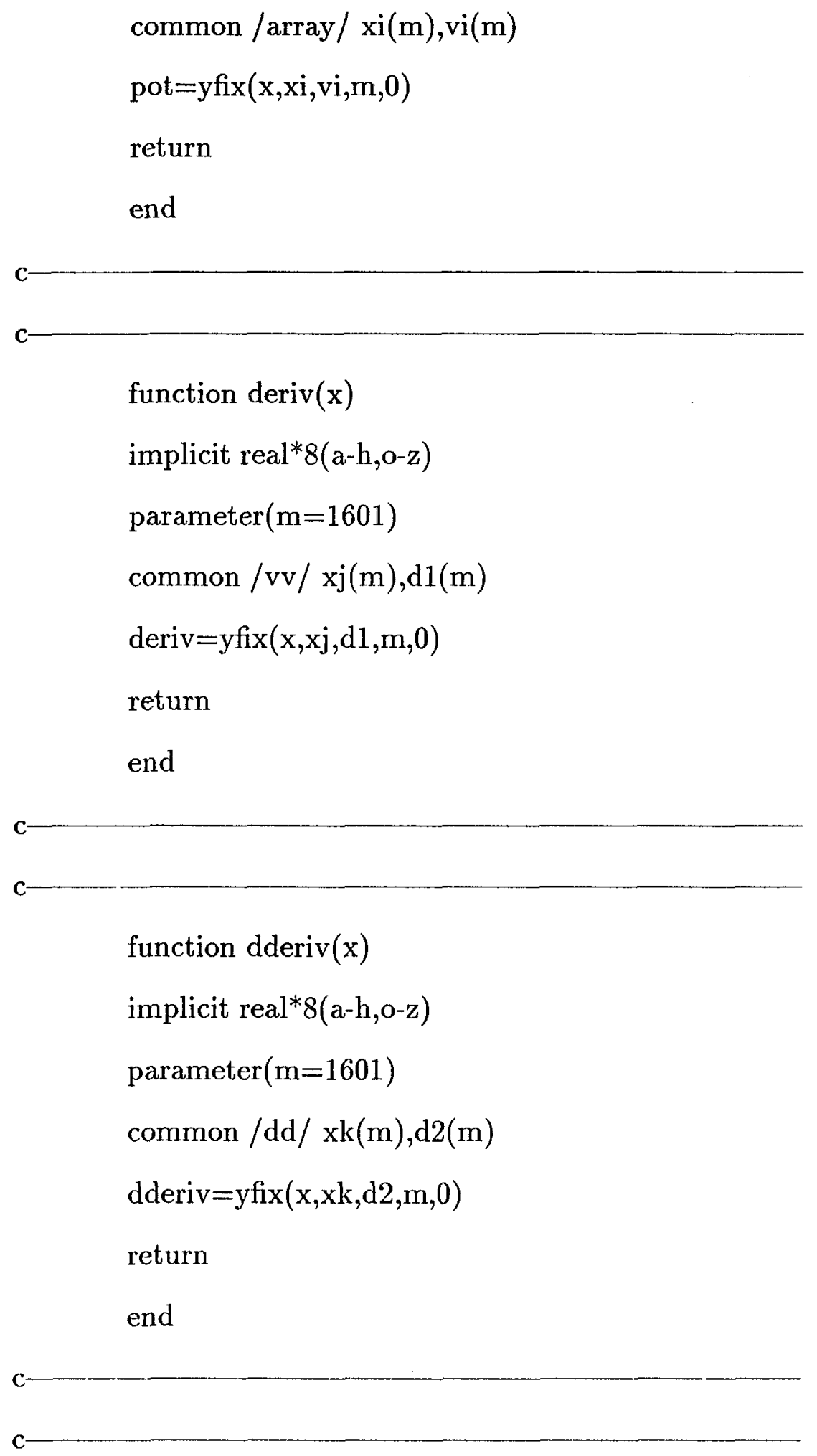


subroutine perlim(xmin,xmax,h,nstep)

implicit real*8(z-h,o-z)

parameter $(1=1601)$

common /ptn/ v(l),v1(l)

common $/ \mathrm{pp} / \mathrm{u}(\mathrm{l}), \mathrm{u} 1(\mathrm{l})$

common /qq/w(l),w1(l)

$\mathrm{nstep}=\operatorname{int}((\mathrm{xmax}-\mathrm{xmin}) / \mathrm{h})$

$\mathrm{x}=\mathrm{xmin}$

$\mathrm{n}=\mathrm{nstep}+1$

$\mathrm{hh}=\mathrm{h} * \mathrm{~h} / 2 . \mathrm{d} 0$

$\mathrm{h} 1=0.5^{*} \mathrm{~h}$

do $1 \mathrm{k}=1$,nstep

$\mathrm{v}(\mathrm{k})=\mathrm{hh}^{*} \operatorname{pot}(\mathrm{x})$

$\mathrm{v} 1(\mathrm{k})=\mathrm{hh} * \operatorname{pot}(\mathrm{x}+\mathrm{h} 1)$

$\mathrm{u}(\mathrm{k})=\mathrm{hh}^{*} \operatorname{deriv}(\mathrm{x})$

$\mathrm{u} 1(\mathrm{k})=\mathrm{hh} * \operatorname{deriv}(\mathrm{x}+\mathrm{h} 1)$

$\mathrm{w}(\mathrm{k})=\mathrm{hh}^{*} \operatorname{dderiv}(\mathrm{x})$

$\mathrm{w} 1(\mathrm{k})=\mathrm{hh} * \operatorname{dderiv}(\mathrm{x}+\mathrm{h} 1)$

$\mathrm{x}=\mathrm{x}+\mathrm{h}$

1 continue

$\mathrm{n}(\mathrm{n})=\mathrm{hh}^{*} \operatorname{pot}(\mathrm{x})$

$\mathrm{u}(\mathrm{n})=\mathrm{hh} * \operatorname{deriv}(\mathrm{x})$

$\mathrm{w}(\mathrm{n})=\mathrm{hh} * \operatorname{dderiv}(\mathrm{x})$

return 
end

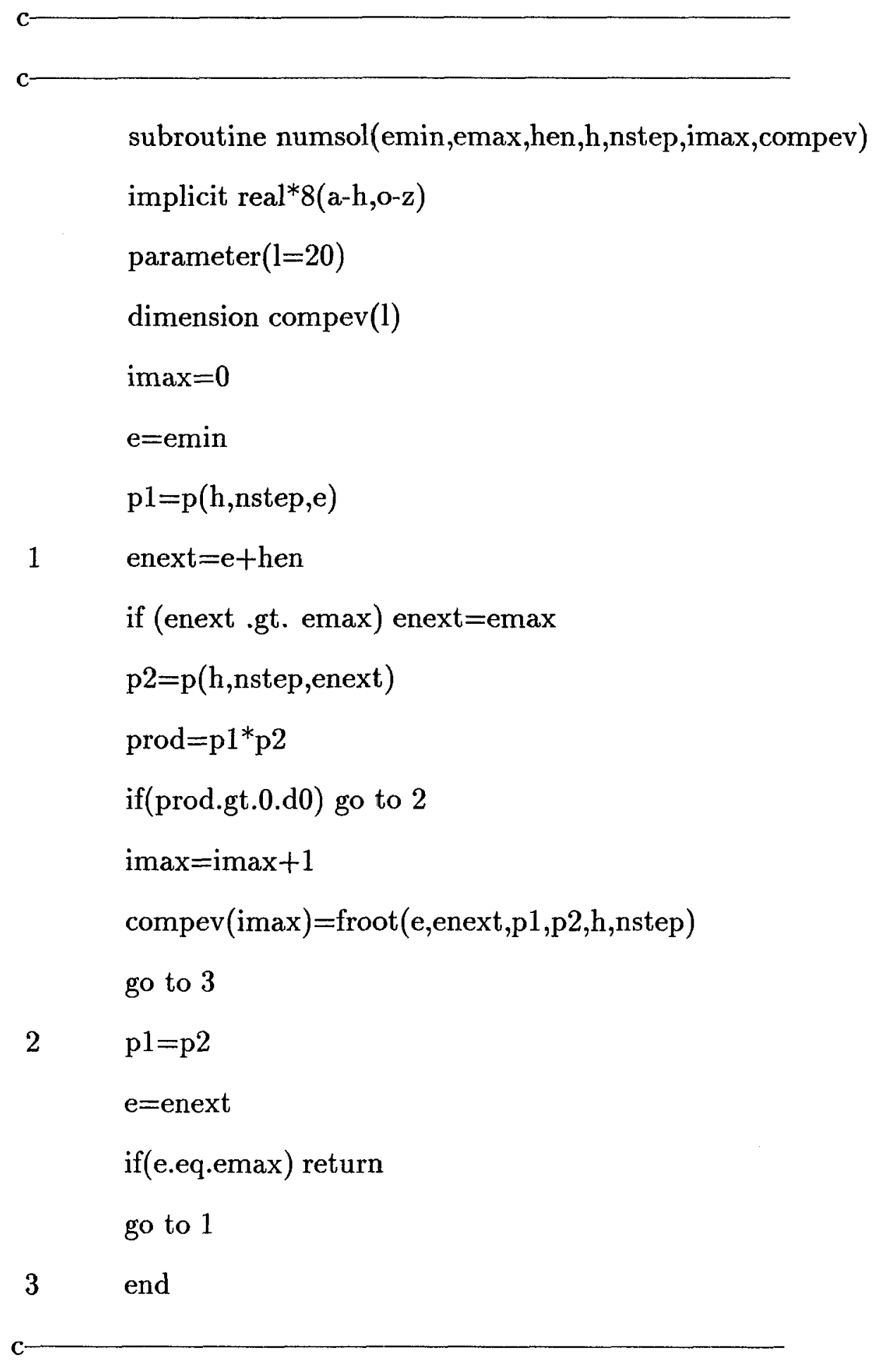




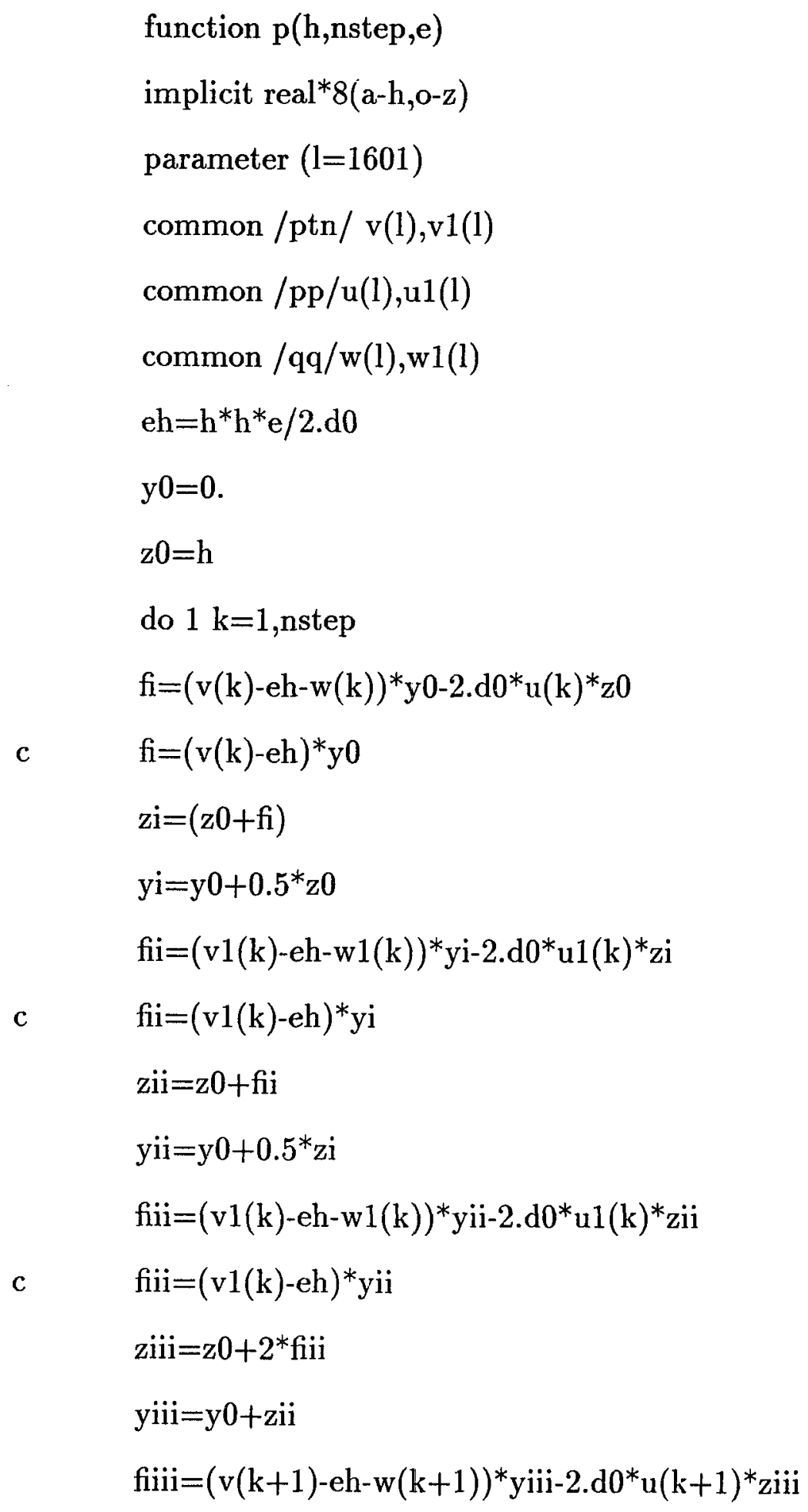




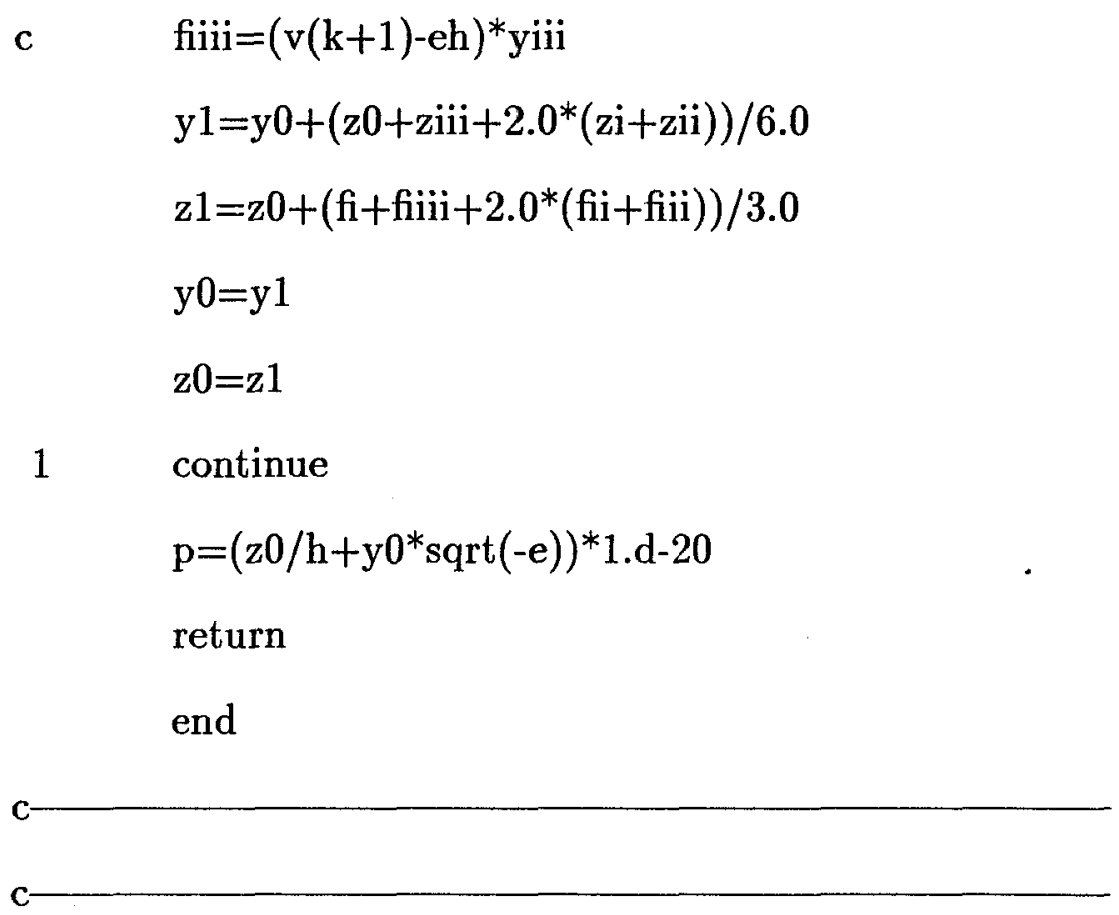

function froot(ea,eb,pa,pb,h,nstep)

implicit real*8(a-h,o-z)

$\mathrm{e} 1=\mathrm{ea}$

$\mathrm{e} 2=\mathrm{eb}$

$\mathrm{p} 1=\mathrm{pa}$

$\mathrm{p} 2=\mathrm{pb}$

tol $=8 . d-05$

$2 \quad$ etest $=0.5 \mathrm{~d} 0 *(\mathrm{e} 1+\mathrm{e} 2)$

ptest $=p(h, n s t e p$, etest $)$

if (ptest.eq.0.d0) go to 4

prod $=p 1 *$ ptest

if (prod.gt.0.d0) go to 1

e2=etest 


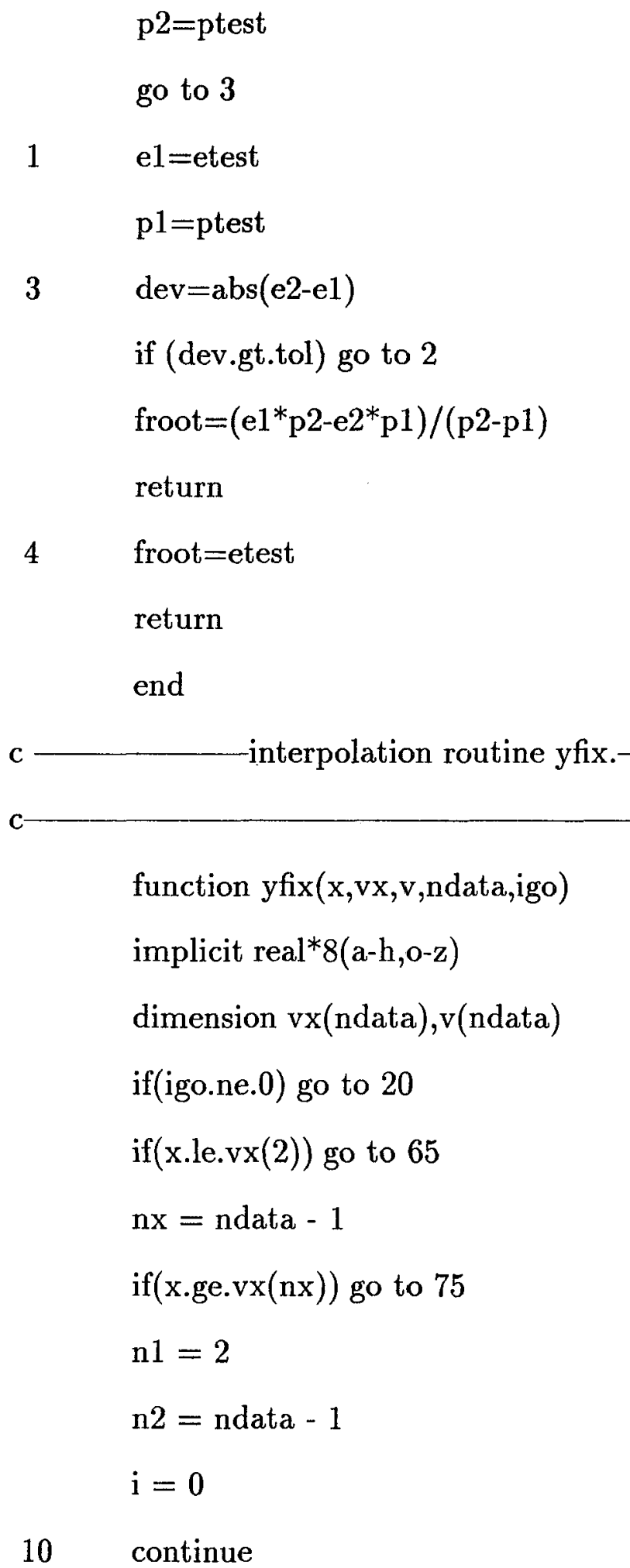

10 continue 
$\mathrm{i}=\mathrm{i}+1$

$\mathrm{j}=(\mathrm{n} 1+\mathrm{n} 2) / 2$

if( $(v x(j) \cdot l e . x)$ go to 15

if(n2-n1 .le. 1) go to 20

$\mathrm{n} 2=\mathrm{j}$

go to 10

15 continue

$\mathrm{n} 1=\mathrm{j}$

if(n2-n1 .le. 1) go to 20

go to 10

20 continue

$$
\begin{aligned}
& \mathrm{n}=\mathrm{n} 2-1 \\
& \mathrm{np}=\mathrm{n} 2 \\
& \mathrm{np} 1=\mathrm{np}+1 \\
& \mathrm{n} 1=\mathrm{n}-1 \\
& \mathrm{x} 0=\mathrm{vx}(\mathrm{n} 1) \\
& \mathrm{y} 0=\mathrm{v}(\mathrm{n} 1) \\
& \mathrm{x} 1=\mathrm{vx}(\mathrm{n}) \\
& \mathrm{y} 1=\mathrm{v}(\mathrm{n}) \\
& \mathrm{x} 2=\mathrm{vx}(\mathrm{np}) \\
& \mathrm{y} 2=\mathrm{v}(\mathrm{np}) \\
& \mathrm{y} 3=\mathrm{v}(\mathrm{np} 1) \\
& \mathrm{q} 0=(\mathrm{x}-\mathrm{x} 1)^{*}(\mathrm{x}-\mathrm{x} 2)^{*}(\mathrm{x}-\mathrm{x} 3) /\left((\mathrm{x} 0-\mathrm{x} 1)^{*}(\mathrm{x} 0-\mathrm{x} 2)^{*}(\mathrm{x} 0-\mathrm{x} 3)\right) \\
& \mathrm{q} 1=(\mathrm{x}-\mathrm{x} 0)^{*}(\mathrm{x}-\mathrm{x} 2)^{*}(\mathrm{x}-\mathrm{x} 3) /\left((\mathrm{x} 1-\mathrm{x} 0)^{*}(\mathrm{x} 1-\mathrm{x} 2)^{*}(\mathrm{x} 1-\mathrm{x} 3)\right)
\end{aligned}
$$




$$
\begin{aligned}
& \mathrm{q} 2=(\mathrm{x}-\mathrm{x} 0)^{*}(\mathrm{x}-\mathrm{x} 1)^{*}(\mathrm{x}-\mathrm{x} 3) /\left((\mathrm{x} 2-\mathrm{x} 0)^{*}(\mathrm{x} 2-\mathrm{x} 1)^{*}(\mathrm{x} 2-\mathrm{x} 3)\right) \\
& \mathrm{q} 3=(\mathrm{x}-\mathrm{x} 0)^{*}(\mathrm{x}-\mathrm{x} 1)^{*}(\mathrm{x}-\mathrm{x} 2) /\left((\mathrm{x} 3-\mathrm{x} 0)^{*}(\mathrm{x} 3-\mathrm{x} 1)^{*}(\mathrm{x} 3-\mathrm{x} 2)\right) \\
& \mathrm{y}=\mathrm{q} 0^{*} \mathrm{y} 0+\mathrm{q} 1^{*} \mathrm{y} 1+\mathrm{q} 2^{*} \mathrm{y} 2+\mathrm{q} 3^{*} \mathrm{y} 3 \\
& \text { go to } 100 \\
& \text { slope }=(\mathrm{v}(2)-\mathrm{v}(1)) /(\mathrm{vx}(2)-\mathrm{vx}(1)) \\
& \mathrm{n} 1=2 \\
& \mathrm{n} 2=3
\end{aligned}
$$$$
\text { go to } 20
$$

66 continue

$$
\mathrm{y}=\operatorname{slope}^{*}(\mathrm{x}-\mathrm{vx}(1))+\mathrm{v}(1)
$$

go to 100

$$
\begin{array}{ll}
75 & \mathrm{nx}=\text { ndata }-1 \\
& \text { slope }=(\mathrm{v}(\text { ndata })-\mathrm{v}(\mathrm{nx})) /(\mathrm{vx}(\text { ndata })-\mathrm{vx}(\mathrm{nx})) \\
& \mathrm{y}=\mathrm{v}(\text { ndata })-\operatorname{slope}^{*}(\mathrm{vx}(\text { ndata })-\mathrm{x}) \\
100 \quad \mathrm{yfix}=\mathrm{y} & \text { return } \\
& \text { end }
\end{array}
$$

c user own library

c interpolation 
c potential

c

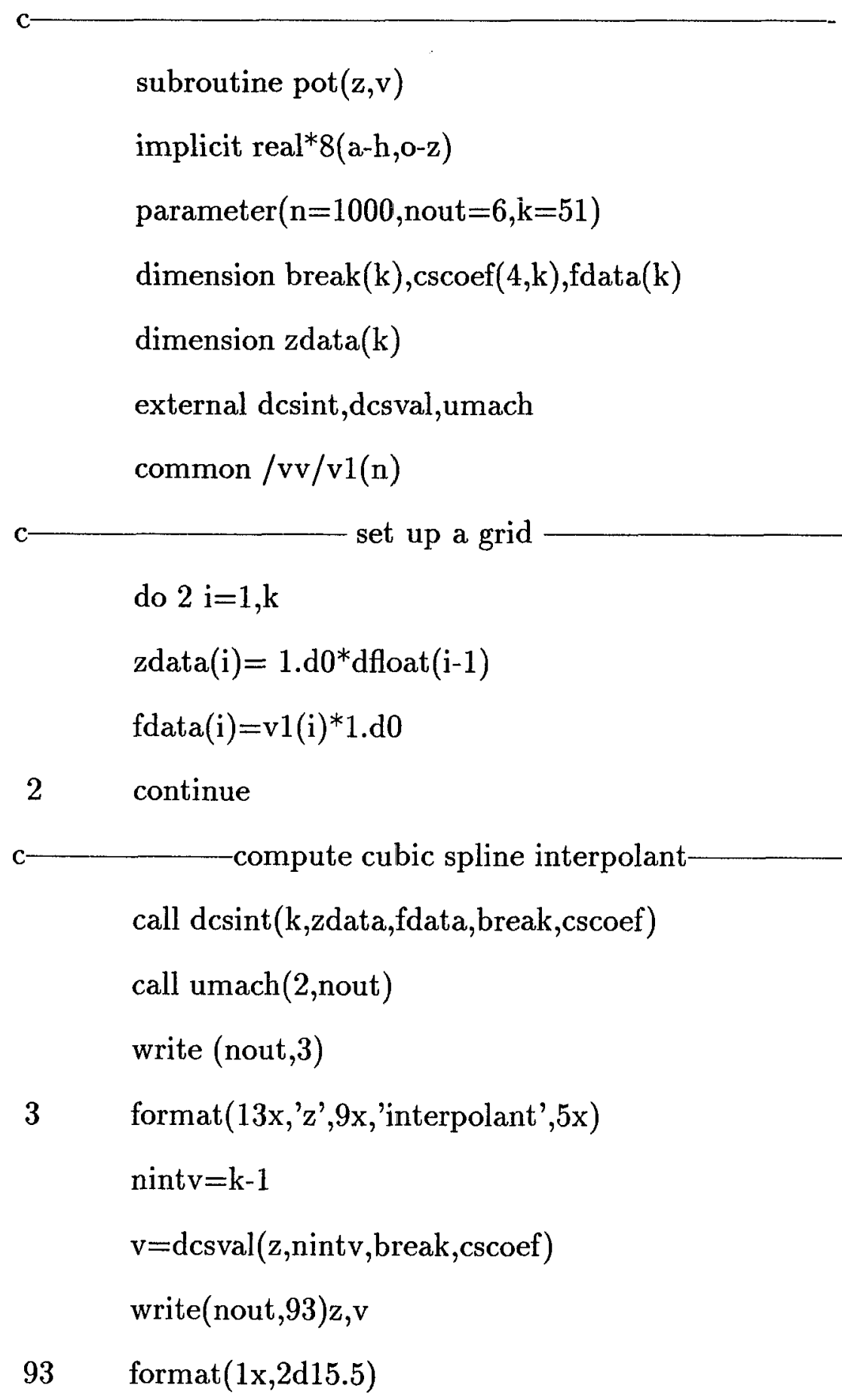




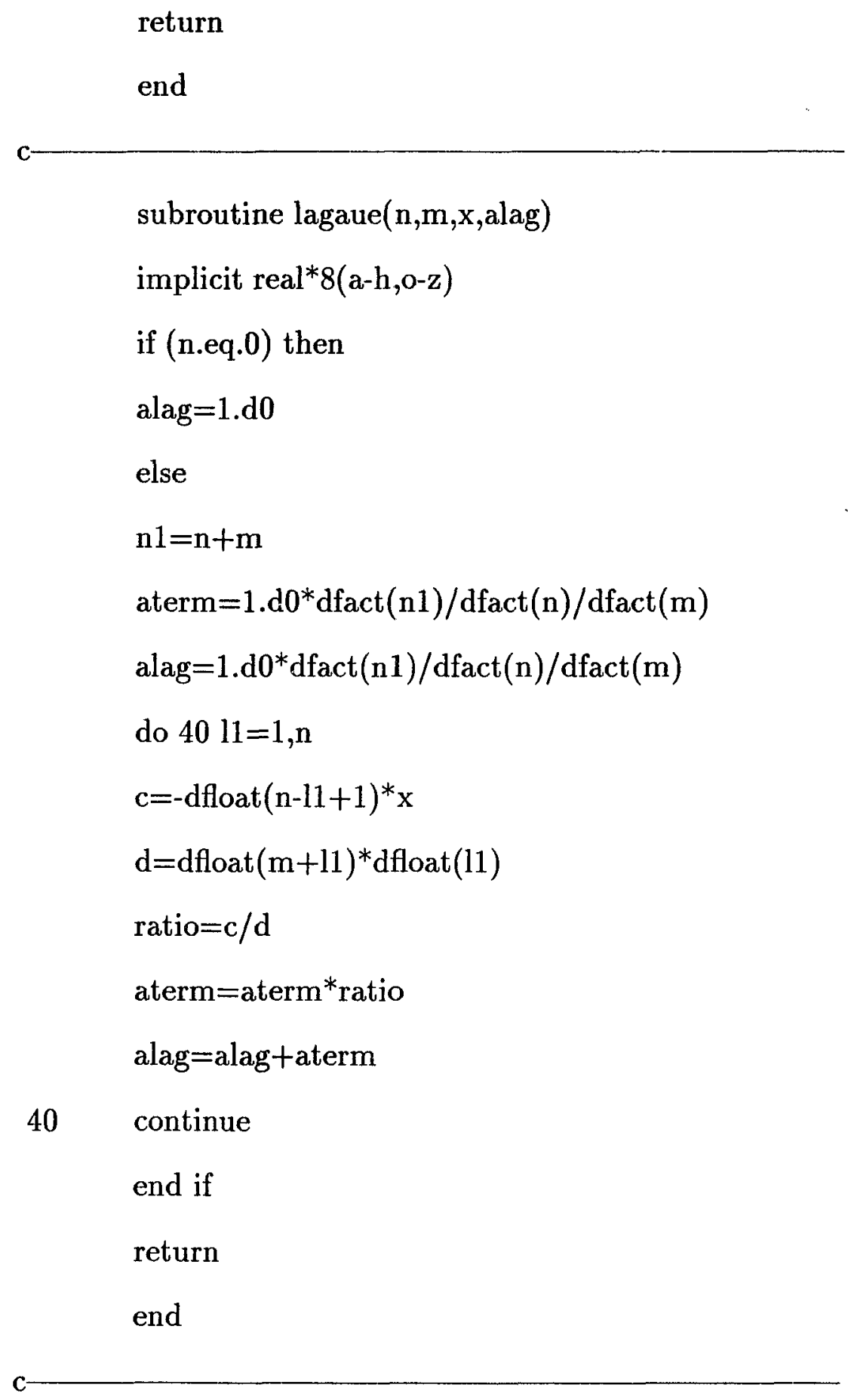

c this program is to create a langdre polynomal 


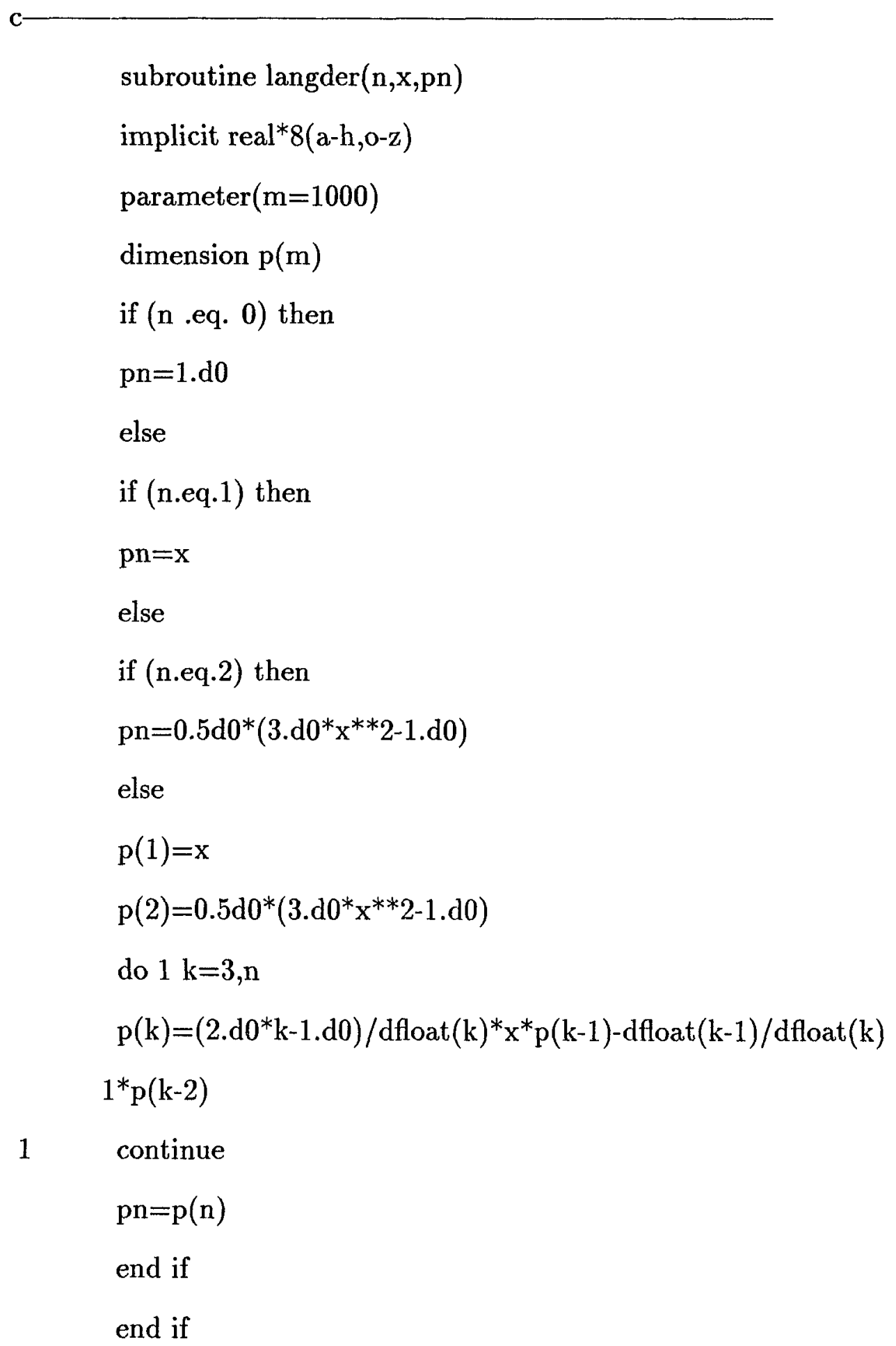


end if

return

end

c-

c this program is to create a jacobi polynomals

subroutine jacobi(n,alfa,beta, $x, a j)$

implicit real $* 8(a-h, o-z)$

parameter $(\mathrm{m}=1000)$

dimension ac $(\mathrm{m})$

if (n.eq.0) then

$a j=1 . d 0$

else

if (n.eq.1) then

$\mathrm{aj}=0.5 \mathrm{~d} 0 *(2 . \mathrm{d} 0 *($ alfa $+1 . d 0)+($ alfa + beta $+2 . \mathrm{d} 0) *(x-1))$

else

if(n.eq.2) then

$\mathrm{aj}=1 \cdot \mathrm{d} 0 / 8 \cdot \mathrm{d} 0^{*}\left(4 \cdot d 0^{*}(\mathrm{alfa}+1 \cdot \mathrm{d} 0)^{*}(\mathrm{alfa}+2 \cdot \mathrm{d} 0)+4 \cdot \mathrm{d} 0^{*}(\right.$ alfa + beta

$1+3 . d 0)^{*}($ alfa $+2 . d 0) *(x-1)+($ alfa + beta $+3 . d 0) *(a l f a+b e t a+4 . d 0)$

$\left.1^{*}(\mathrm{x}-1)^{* *} 2\right)$

else

$\operatorname{ac}(1)=0.5 \mathrm{~d} 0 *(2 . \mathrm{d} 0 *(\operatorname{alfa}+1 . \mathrm{d} 0)+($ alfa + beta $+2 . \mathrm{d} 0) *(\mathrm{x}-1))$ 
$\operatorname{ac}(2)=1 \cdot d 0 / 8 \cdot d 0 *(4 \cdot d 0 *(\operatorname{alfa}+1 \cdot d 0) *(a l f a+2 . d 0)+4 \cdot d 0 *(a l f a+b e t a$ $1+3 . \mathrm{d} 0)^{*}(\text { alfa }+2 . \mathrm{d} 0)^{*}(\mathrm{x}-1)+(\text { alfa }+ \text { beta }+3 . \mathrm{d} 0)^{*}($ alfa + beta $+4 . \mathrm{d} 0)$ $\left.1^{*}(\mathrm{x}-1)^{* *} 2\right)$

do $1 \mathrm{i}=\mathbf{3 , n}$ $\operatorname{ac}(i)=1 . d 0 / 2 . d 0 /$ dfloat $(i) /(1 . d 0 * i+a l f a+b e t a) /(2 . d 0 * i+a l f a-2 . d 0$ $1+$ beta $) *((2 . d 0 * i+$ alfa + beta- $1 . d 0) *((2 . d 0 * i+$ alfa + beta $) *(2 . d 0 * i+a l f a+$ 1beta-2.d0 $)^{*} \mathrm{x}+\left(\right.$ alfa $^{* *} 2-$ beta $\left.\left.^{* *} 2\right)\right)^{*}$ ac $(\mathrm{i}-1)-2 . \mathrm{d} 0^{*}\left(1 . \mathrm{d} 0^{*} \mathrm{i}+\text { alfa-1.d } 0\right)^{*}$ $1(1 . \mathrm{d} 0 * \mathrm{i}+$ beta-1.d0)*(2.d0*i+alfa + beta $) * a c(i-2))$

continue

$a j=a c(n)$

end if

end if

end if

return

end

c-

c integrate the differential equation at any given e by

c numerov method to contruct $\mathrm{p}(\mathrm{e})$.

subroutine perlim(xmin, $x \max , \mathrm{h}, \mathrm{nstep})$

implicit real*8(a-h,o-z $)$

parameter $(1=1500)$ 


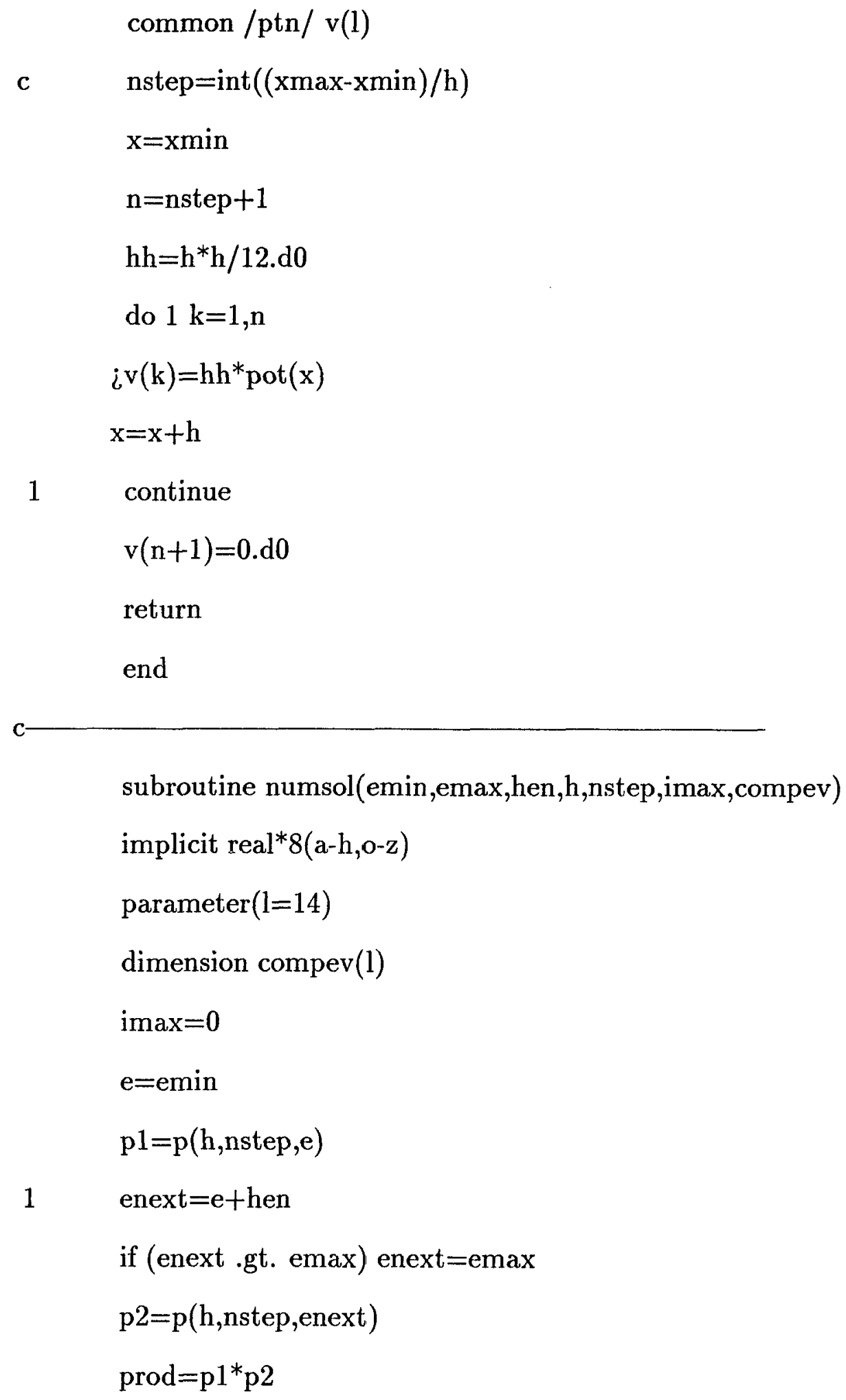




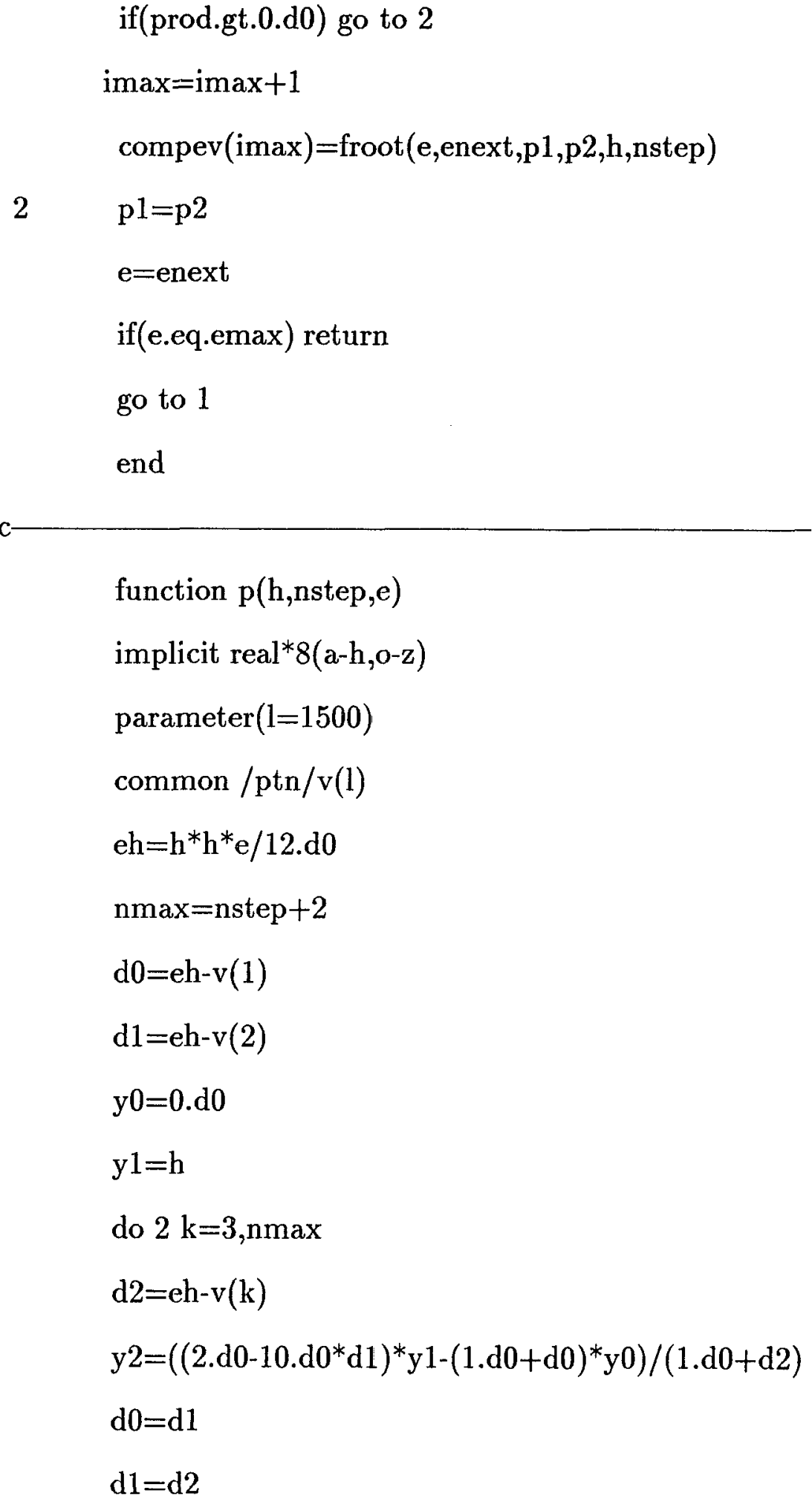




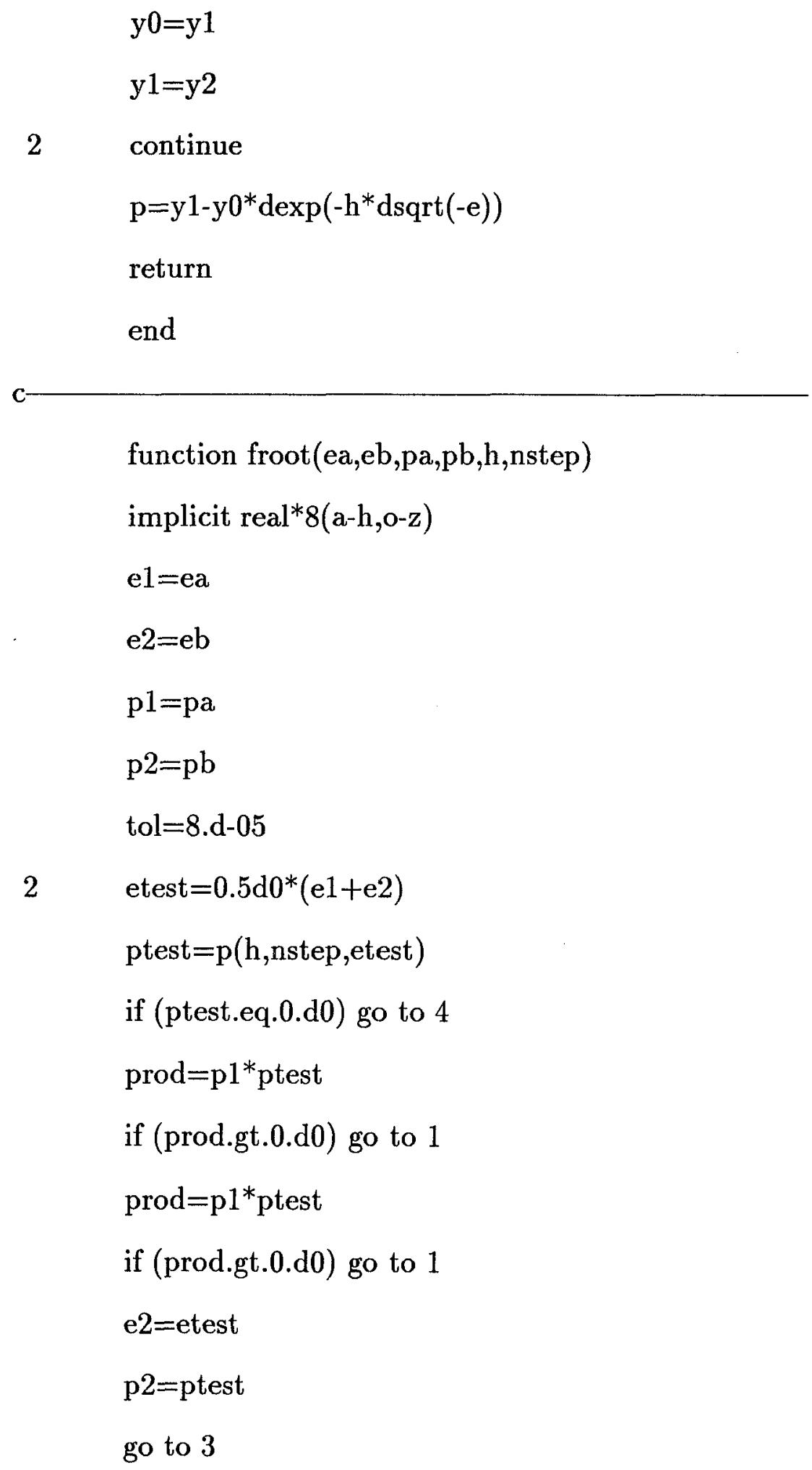




$\begin{array}{ll}1 & \text { e1=etest } \\ & \text { p1=ptest } \\ 3 & \text { dev=abs }(\mathrm{e} 2-\mathrm{e} 1) \\ & \text { if }(\text { dev.gt.tol }) \text { go to } 2 \\ & \text { froot }=\left(\mathrm{e} 1^{*} \mathrm{p} 2-\mathrm{e} 2^{*} \mathrm{p} 1\right) /(\mathrm{p} 2-\mathrm{p} 1) \\ & \text { return } \\ & \text { froot }=\text { etest } \\ & \text { return } \\ & \text { end }\end{array}$




\section{VITA}

Lijun Zhang was born on Nov. 29, 1956. She received her M.S. in Physics in 1988, M.S. in System Science in 1992 and Ph.D. in Physics in 1993 from Louisiana State University. Her publications include:

[1] A. R. P. Rau and L. Zhang: "Mapping degenerate perturbation in atoms onto an asymmetric rotor", Phys. Rev. A 42, 6342 (1990).

[2] L. Zhang and A. R. P. Rau, "Electron-pair analysis for doubly excited ridge states", Phys. Rev. A Dec. (1992).

[3] L. Zhang and A. R. P. Rau, "Electron-pair analysis for doubly excited ridge states II: $\mathrm{L}=1$ ", in preparation. 


\section{DOCTORAL EXAMINATION AND DISSERTATION REPORT}

Candidate: Lijun Zhang

Major Field: Physics

Title of Dissertation: Electron-Pair Analysis for Doubly Excited Ridge States

\section{Approved:}

Hawifraksh Pan

Majof Professor and Chairman

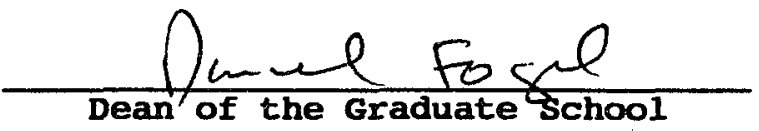

EXAMINING COMMITTEE:

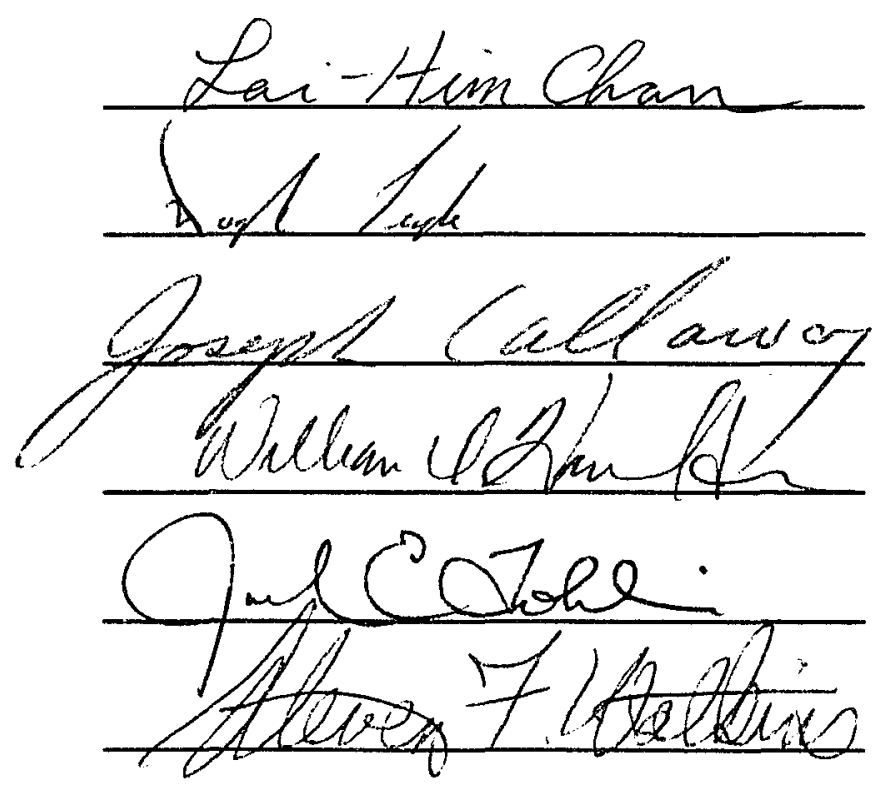

Date of Examination:

6 November 1992 